\title{
A Study of Magnetic Reconnection: From 2D Energy Release to 3D Spreading and Localization
}

\author{
Lucas S. Shepherd \\ West Virginia University
}

Follow this and additional works at: https://researchrepository.wvu.edu/etd

\section{Recommended Citation}

Shepherd, Lucas S., "A Study of Magnetic Reconnection: From 2D Energy Release to 3D Spreading and Localization" (2014). Graduate Theses, Dissertations, and Problem Reports. 204.

https://researchrepository.wvu.edu/etd/204

This Dissertation is protected by copyright and/or related rights. It has been brought to you by the The Research Repository @ WVU with permission from the rights-holder(s). You are free to use this Dissertation in any way that is permitted by the copyright and related rights legislation that applies to your use. For other uses you must obtain permission from the rights-holder(s) directly, unless additional rights are indicated by a Creative Commons license in the record and/ or on the work itself. This Dissertation has been accepted for inclusion in WVU Graduate Theses, Dissertations, and Problem Reports collection by an authorized administrator of The Research Repository @ WVU.

For more information, please contact researchrepository@mail.wvu.edu. 


\title{
A Study of Magnetic Reconnection: From 2D Energy Release to 3D Spreading and Localization
}

\author{
Lucas S. Shepherd
}

\author{
Dissertation submitted to the \\ Eberly College of Arts and Sciences \\ at West Virginia University \\ in partial fulfillment of the requirements \\ for the degree of \\ Doctor of Philosophy \\ in \\ Physics \\ Paul Cassak, Ph.D., Chair \\ Earl Scime, Ph.D. \\ Duncan Lorimer, Ph.D. \\ D.J. Pisano, Ph.D. \\ Arun Ross, Ph.D. \\ Department of Physics and Astronomy \\ Morgantown, West Virginia \\ 2014
}

Keywords: Magnetic Reconnection; Plasma Copyright 2014 Lucas S. Shepherd 


\title{
ABSTRACT \\ A Study of Magnetic Reconnection: \\ From 2D Energy Release to \\ 3D Spreading and Localization
}

\author{
Lucas Seth Shepherd
}

Magnetic reconnection is a plasma process in which stored magnetic energy is converted into thermal and kinetic energies of the surrounding plasma. Oppositely directed magnetic field lines break and cross connect due to a dissipative mechanism. The now bent, reconnected field lines retreat from the X-line (the location of reconnection) at the Alfven speed due to the magnetic tension in the reconnected magnetic field, therefore generating outflows. This dissertation addresses three fundamental properties of magnetic reconnection.

Solar flares are explosive events in the solar corona in which magnetic reconnection mediates the rapid release (on the order of minutes) of energy stored in magnetic fields into the surrounding plasma. The Sweet-Parker (collisional) model was the first self-consistent theory to explain magnetic reconnection, but is far too slow to explain observations. The formation of secondary islands make Sweet-Parker reconnection faster, but is it fast enough to explain energy release rates? Collisionless (Hall) reconnection leads to energy release rates fast enough to explain observations. Large-scale resistive Hall-Magnetohydrodynamics simulations of the transition from Sweet-Parker to Hall reconnection are presented; the first to separate secondary islands from collisionless effects. Three main results are described. There exists a regime with secondary islands but without collisionless effects entering, and the reconnection rate is faster than Sweet-Parker, but significantly slower than Hall reconnection. This implies that secondary islands do not cause the fastest reconnection rates. The onset of Hall reconnection ejects secondary islands from the vicinity of the X-line, implying that energy is released more rapidly during Hall reconnection.

Early models of magnetic reconnection have treated reconnection as twodimensional. However, naturally occurring magnetic reconnection often begins in a localized region and spreads in the direction perpendicular to the plane of reconnection. Theoretical arguments and large-scale two fluid simulations are used to study the spreading of reconnection X-lines localized in the direction of the current as a function of the strength of the out-of-plane (guide) magnetic field. It is found that the mechanism causing the spreading is different for weak and strong guide fields. In the weak guide field limit, spreading is due to the motion of the current carriers. However, spreading for strong guide fields is bidirectional and is due to 
the excitation of Alfvén waves along the guide field. In general, we suggest that the $\mathrm{X}$-line spreads bidirectionally with a speed governed by the faster of the two mechanisms for each direction. A prediction of the strength of the guide field at which the spreading mechanism changes is formulated and verified with three-dimensional simulations.

In the solar wind, magnetic reconnection exhausts measuring 600 [Gosling et al. (2007)] and 390 [Phan et al. (2006)] Earth radii in length have been observed. The authors assumed that the extended exhaust was caused by an extended X-line. If this is the case, what mechanism is responsible for these large scale structures? It has been suggested these structures are formed by a small X-line forming near the sun and spreading as the X-line convects away from the sun. Another possibility is the $\mathrm{X}$-line is localized in a small region and the exhaust expands into the outof-plane direction. Theoretical arguments and large-scale simulations are used to study localized (not spreading) magnetic reconnection, and its three-dimensional structure. Localized reconnection may also be vital to the formation of supraarcade downflows (SADs) in the corona. Both solar wind and coronal applications are discussed. 


\section{Acknowledgments}

As my time at WVU comes to a close, I am very grateful and thankful for all of the people who have had a significant impact in my life. First and foremost, I thank my parents, Lloyd and Donna Shepherd, who instilled in me the drive to continue my education and for their support over this long journey. To my Aunt and Uncle, Betsy and Steve Helmick, for their unending support and advice along the way, even though I may have not always heeded it. My love goes out to the rest of my family who have supported me over these many years - Anna Mae, Uncle Jack, Grandma and Grandpa Snyder, and Grandma and Grandpa Shepherd. Also, my future wife Nicole, who kept me on task and supported me as I finished this dissertation. Her patience in dealing with me over the past few months is unmatched.

I am very grateful for Paul Cassak, my adviser. He was always supportive and very patient during my time working with him. He gave me the tools to truly succeed and understand physics. I would also like to thank Earl Scime for taking a chance on me. I am forever thankful for the opportunity you have given me. I would also like to thank the rest of the committee Duncan Lorimer, D.J. Pisano, and Arun Ross for sitting on my committee.

I owe a special dedication to three individuals whose friendship I will always cherish. Jerry Carr, one of my greatest friends, we began our journey together nearly seven years ago over a discussion of football. I cannot think of a better person to have as a friend. I want to thank Mitch Mickaliger for all of the late night video game marathons and Minneo's runs. Finally, Spencer Wolfe for answering all of my dumb questions I had and for being a good roommate for many years. Without the three of you, I do not know what kind of person I would be today.

I would also to make a call out to the folks who have enriched my life and contributed to my success at WVU and beyond - Colin Komar, Dusty McCarren, Katie Rabidoux, Andrew Seymour, Matt Galante, Nishal Varang, Paul Miller, and Ray Fermo. Thank you! 


\section{Table of Contents}

List of Figures $\quad$ vii

1 Introduction 1

1.1 Magnetic Reconnection: A Brief History . . . . . . . . . . . . . . . . 1

1.2 Magnetic Reconnection Physics in Four Regimes . . . . . . . . . . . . 5

1.2.1 Sweet-Parker Reconnection . . . . . . . . . . . . . . . . . . 9 9

1.2.2 Sweet-Parker Enhanced by Secondary Islands . . . . . . . . . 11

1.2.3 Hall Reconnection . . . . . . . . . . . . . . . . . 13

1.2.4 Anomalous Resistivity . . . . . . . . . . . . . . . . 18

1.3 MHD Discontinuities ... . . . . . . . . . . . . . 18

1.3.1 Contact Discontinuity (CD) . . . . . . . . . . 21

1.3.2 Tangential Discontinuity (TD) . . . . . . . . . . . . 22

1.3.3 Rotational Discontinuity (RD) . . . . . . . . . . . . 22

1.3.4 MHD Shocks . . . . . . . . . . . . . . . 23

1.4 Summary of Research . . . . . . . . . . . . . . . . . 25

2 The transition between Sweet-Parker with secondary islands to Hall reconnection 29

2.1 Introduction . . . . . . . . . . . . . . . . . . 30

2.2 Simulation Setup . . . . . . . . . . . . . . . . . . . . . . . . . . . . . . . . . . . . . 33

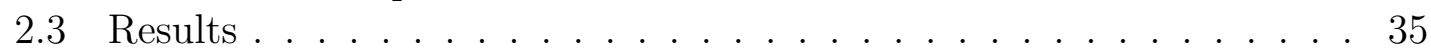

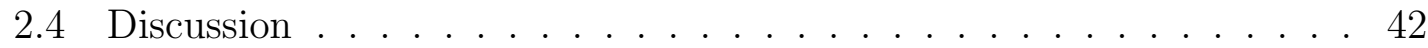

3 Guide Field Dependence of 3D X-line Spreading During Collisionless Magnetic Reconnection 44

3.1 Introduction . . . . . . . . . . . . . . . . . . . . 45

3.2 Theory . . . . . . . . . . . . . . . . 50

3.3 Simulation Setup . . . . . . . . . . . . . . . . . . . . . . . . . . . . . . . . . . . 55

3.4 Results ......................... 60

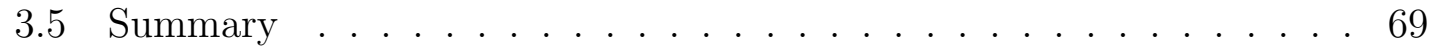

4 Structure of Reconnection Exhaust $\quad 72$

4.1 Introduction . . . . . . . . . . . . . . . . . . 72

4.2 Theory ............................... 75

4.3 Simulation Setup . . . . . . . . . . . . . . . 84

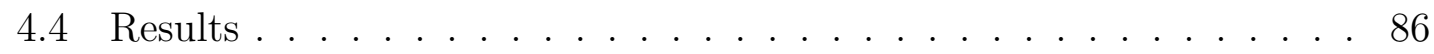

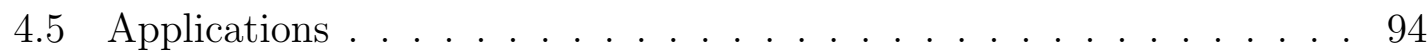

4.5.1 Solar Wind . . . . . . . . . . . . . . . . . . 94

4.5.2 Supra-Arcade Downflows . . . . . . . . . . . . . . . 98

4.6 Conclusion . . . . . . . . . . . . . . . . . . 100 
5 Summary of Work 102

5.1 Summary of Results . . . . . . . . . . . . . . . . . . . 102

5.1.1 Role of Secondary Islands in Energy Release . . . . . . . . . . 103

5.1 .2 Guide Field Dependence of X-line Spreading . . . . . . . . . . 104

5.1.3 X-line Structure for Localized Reconnection . . . . . . . . . . 106 


\section{List of Figures}

1.1 A solar flare with coronagraph . . . . . . . . . . . . . . . . . . 2

1.2 Simple magnetic reconnection sketch . . . . . . . . . . . 3

1.3 Petschek reconnection sketch . . . . . . . . . . . . . . . 4

1.4 Secondary islands during Sweet-Parker . . . . . . . . . . . . . . . 12

1.5 Quadropolar structure of Hall reconnection . . . . . . . . . . . . 16

1.6 Uniform versus anomalous resistivity . . . . . . . . . . . . . 19

1.7 Contact and tangential discontinuities diagram . . . . . . . . . . 22

1.8 Rotational discontinuity and MHD shocks . . . . . . . . . 25

2.1 Reconnection rate and current sheet thickness as a function of time . 36

2.2 Current sheet evolution with one secondary island . . . . . . . . . . 37

2.3 Current sheet evolution with multiple islands . . . . . . . . . . . . 39

2.4 Hall term as a function of time . . . . . . . . . . . . . . . . 41

3.1 Sketch of reconnection spreading . . . . . . . . . . . . 45

3.2 Dawn-dusk asymmetry in the magnetotail . . . . . . . . . . 46

3.3 Reconnection spreading in the laboratory . . . . . . . . . . . 48

3.4 Two-ribbon flare evolution . . . . . . . . . . . . . . . . . . . . 49

3.5 Schematic of the different types of reconnection spreading . . . . . . 54

3.6 3D reconnection initial perturbation . . . . . . . . . . . . . 59

3.7 Hall reconnetion spreading . . . . . . . . . . . . . . . . 61

3.8 Reconnection spreading dependence on guide field . . . . . . . . . . 62

3.9 X-line length calculation . . . . . . . . . . . . . . . . . . 65

3.10 Spreading speed versus guide field strength . . . . . . . . 66

4.1 Large-scale reconnection site schematic . . . . . . . . . . . 73

4.2 TRACE image of SADs . . . . . . . . . . . . . 75

4.3 2D reconnection MHD discontinuities . . . . . . . . . . . 77

4.4 Predicted 3D exhaust structure . . . . . . . . . . . . . . . . 79

4.5 3D reconnection geometry $\ldots \ldots \ldots \ldots \ldots$

4.6 3D discontinuity structure $\ldots \ldots \ldots \ldots \ldots$

4.7 3D in-plane reconnection geometry $\ldots \ldots \ldots \ldots . \ldots . \ldots$

4.8 Localized versus spreading reconnection . . . . . . . . . . . . . 87

4.9 Guide field dependence on opening angle . . . . . . . . . . . . . 88

4.10 Opening angle versus guide field strength . . . . . . . . . . . . . 89

4.11 Collimation as a function of guide field . . . . . . . . . . . 90

$4.123 \mathrm{D}$ reconnection rates . . . . . . . . . . . . . . . . 90

4.13 Collimation versus X-line length . . . . . . . . . . . . . . . 91

4.14 In-plane discontinuity structure . . . . . . . . . . . . . . . . 92

4.15 Exhaust structure in $3 \mathrm{D} \ldots \ldots . \ldots . \ldots . \ldots 93$

4.16 Sketch of exhaust in the $x y$ and $x z$ plane $\ldots \ldots \ldots \ldots$. . . . 96

$4.173 \mathrm{D}$ view of the reconnection exhaust with density depletion . . . . . 99 


\section{Chapter 1}

\section{Introduction}

\subsection{Magnetic Reconnection: A Brief History}

Solar flares are large explosive events that release a huge amount of radiation and solar material into interplanetary space that were first observed by Carrington and Hodgson in 1859 [Carrington (1859); Hodgson (1859)]. A solar flare is shown in Fig. 1.1(a) in the ultraviolet light range and corresponds to a temperature of 1.5 million Kelvin (195 $\AA$ ). The flare is located just above the center of the image as the very bright spot. Solar flares release a large amount of energetic particles into interplanetary space. This can be seen in Fig. 1.1(b) as the "snow" that appears in the images, which is caused by energetic particles impacting the observation tool. Solar flares within a time scale on the order of a few minutes to a few hours, and release up to $10^{32}$ ergs of energy. What accounts for this rapid energy release? Because the dynamics in the solar corona are controlled by magnetic fields, a possible mechanism is magnetic diffusion given by

$$
\frac{\partial \boldsymbol{B}}{\partial t} \sim \frac{\eta c^{2}}{4 \pi} \nabla^{2} \boldsymbol{B},
$$

which comes from electron-ion collisions as described by the induction equation, which is discussed in Sec. 1.2. Equation 1.1 states the rate of change of the magnetic 

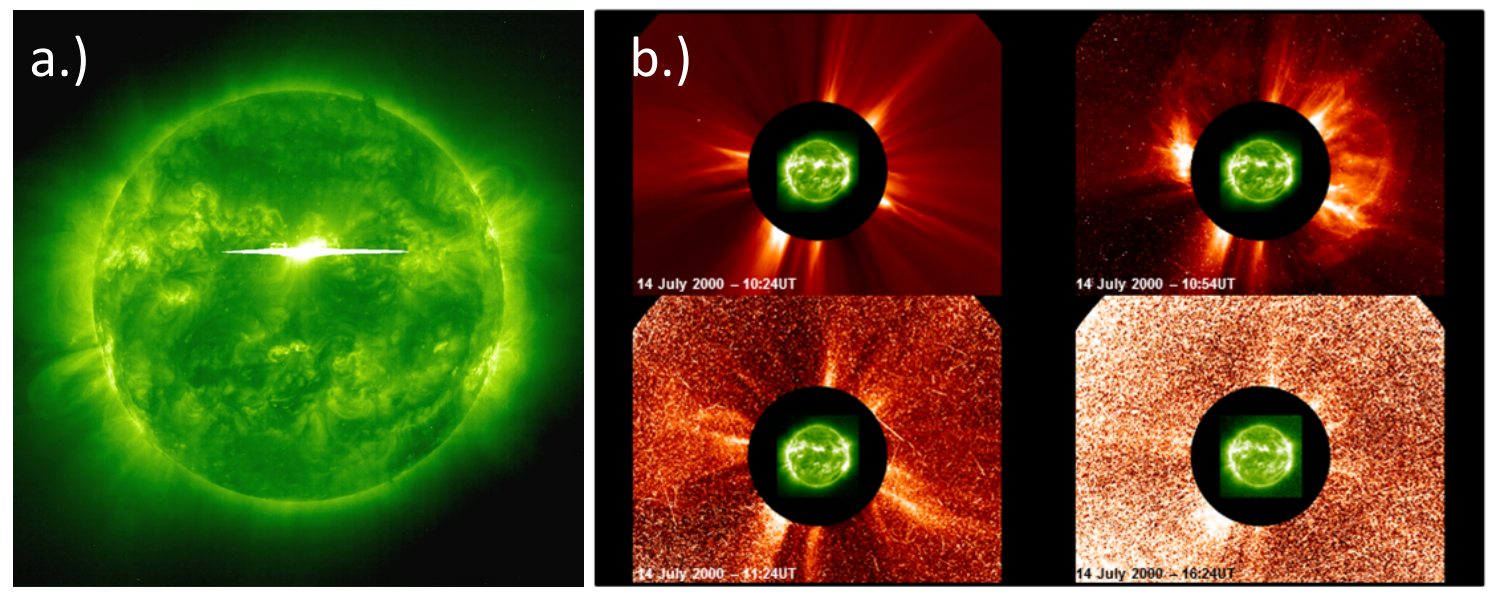

Figure 1.1: a.) Image of solar flare taken on July 14, 2000, known as the Bastille Day Flare. The very bright spot in the center of the image is the location of flare and is caused by energization of particles. b.) Image of the solar corona (with the main body of the sun blocked out). A stream of energetic particles hits the coronagraph quickly after the flare. The snow in the image is energetic particles impacting the coronagraph. Image Credit: NASA/SOHO

field $(\boldsymbol{B})$ is equal to the diffusion of magnetic fields due to resistivity $(\boldsymbol{\eta})$. A scaling analysis $\left(\nabla \rightarrow 1 / L\right.$ and $\left.\partial / \partial t \rightarrow 1 / t_{D}\right)$ of Eq.1.1 reveals the time it takes for a coronal loop of size $L$ to diffuse away due to resistivity is $t_{D} \sim 4 \pi L^{2} / \eta c^{2}$. The radius of coronal flux ropes are on the order $L \sim 10^{9} \mathrm{~cm}$ and assuming a resistivity of $\eta \sim 1 \times 10^{-16} \mathrm{~s}$, yields a time scale roughly $t_{D} \sim 1.4 \times 10^{14} \mathrm{~s}$ or about 4 million years! It is quite evident that magnetic diffusion is not the mechanism responsible for energy release in solar flares.

A more likely mechanism is magnetic reconnection. Magnetic reconnection is a plasma process which converts stored magnetic energy into thermal and kinetic energies of the surrounding plasma. Reconnection was first described by graduate student James Dungey. Dungey found magnetic field lines that are anti-parallel would break and cross connect due to a diffusive mechanism (e.g., resistivity) [Dungey 


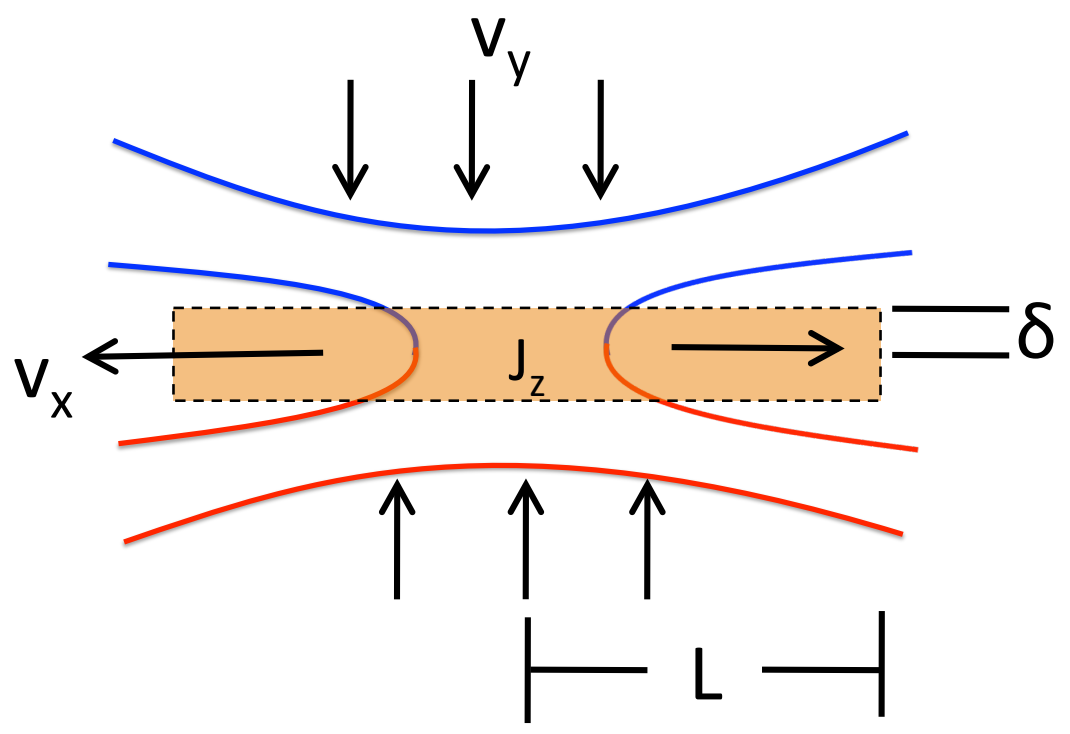

Figure 1.2: A simple sketch of magnetic reconnection. Oppositely directed magnetic fields in red and blue are convected towards each other by $v_{y}$ and proceed to break and reconnect, which generates outflows $v_{x}$. The oppositely directed magnetic fields generate an out-of-page current $J_{z}$.

$(1953,1958)]$. A thin current sheet forms between the regions of oppositely directed field lines. A general sketch of the reconnection process can be seen in Fig. 1.2.

Peter Sweet presented the first self-consistent model of magnetic reconnection [Sweet (1958)]. Sweet modeled a solar flare as two regions of bipolar magnetic field that come together. In this process, two regions of oppositely directed magnetic field lines are driven together and magnetic reconnection occurs where magnetic energy is released and is converted into plasma flows towards the end of the current sheet. A scaling analysis performed by Eugene Parker of Sweet's reconnection model [Parker (1957)] using resistive magnetohydrodynamics predicted a sizeable energy release time of about $t \sim 10^{7} \mathrm{~s}$ or about 4 months. This energy release process is much faster than diffusion, but still not fast enough to account for energy release times on the order of minutes. 


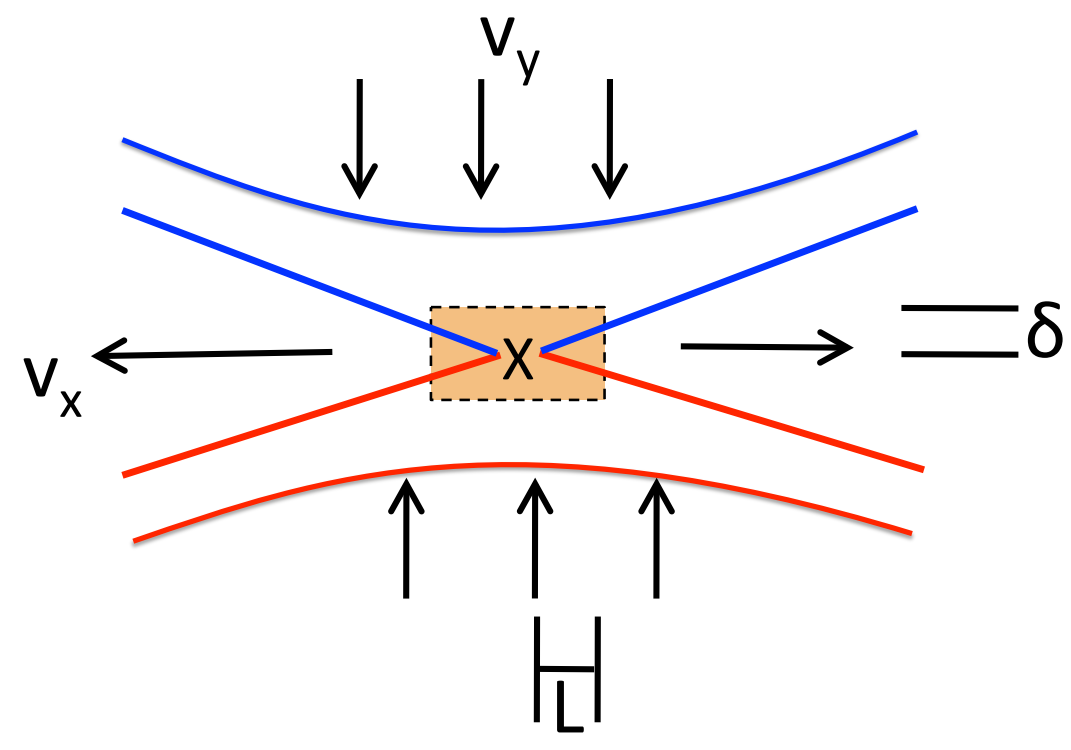

Figure 1.3: A sketch of Petschek magnetic reconnection. Oppositely directed magnetic fields in red and blue are convected towards each other by $v_{y}$ and proceed to break and reconnect at the X-point. The current sheet is much shorter than Sweet-Parker and opens up into a X-type configuration.

The Sweet-Parker model does not solve the problem of very fast energy release rates. A modification of the Sweet-Parker model involved replacing the long, thin current sheet with a much smaller, thin current sheet [Petschek (1964)], as seen in Fig. 1.3. The energy release times are fast enough because the current layer has an open (X-type) configuration, where only a small amount of the plasma is required to pass through the much smaller current sheet. The energy release is mediated by standing slow shocks that are created by bent field lines. It was believed that the energy release problem had a proper solution, but Biskamp (1986) discovered that a smaller, thin current sheet cannot maintain an open configuration without a spatially non-uniform resistivity[Biskamp (1986)], also known as anomalous resistivity.

The X-type configuration allows for energy release times consistent with the observations of solar flare release times. The question remains, what mechanism is 
responsible for the X-type configuration? Let us consider non-MHD effects. It was found that when the Hall term is activated the X-type reconnection layer is realized with energy release rates that are fast enough, as shown by the GEM Challenge [Birn et al. (2001)]! Instead of augmenting the local resistivity, the Hall effect employs dispersive waves that create the open X-type geometry [Mandt et al. (1994); Rogers et al. (2001)], and is entirely self-consistent. It should be noted that the idea that dispersive waves cause the open X-type geometry is not universally accepted. For example, it has been proposed that the X-type geometry is created by secondary islands continously generated in the current sheet which maintains a small lengthscale current sheet [Daughton et al. (2006)].

\subsection{Magnetic Reconnection Physics in Four Regimes}

Much can be learned about reconnection using the magnetohydrodynamics (MHD) model. The governing equations for ideal-MHD are as follows. Faraday's law is given by

$$
\frac{\partial \boldsymbol{B}}{\partial t}=-c \boldsymbol{\nabla} \times \boldsymbol{E}
$$

The continuity equation, which describes mass conservation, is

$$
\frac{\partial n}{\partial t}+\nabla \cdot(n \boldsymbol{v})=0
$$


The momentum equation of the bulk of the plasma is described by

$$
m_{i} n\left(\frac{\partial \boldsymbol{v}}{\partial t}+(\boldsymbol{v} \cdot \nabla) \boldsymbol{v}\right)=-\nabla P+\frac{\boldsymbol{J} \times \boldsymbol{B}}{c}
$$

Only the ion mass is considered because the bulk of the mass is held by the ions. Ampere's Law is

$$
\boldsymbol{\nabla} \times \boldsymbol{B}=\frac{4 \pi}{c} \boldsymbol{J}
$$

Ohm's Law is

$$
\boldsymbol{E}+\frac{\boldsymbol{v} \times \boldsymbol{B}}{c}=0
$$

Adding or removing terms from Ohm's Law (e.g., resistivity and the Hall term) alters the reconnection dramatically, as we will demonstrate later. Gauss' Law of magnetism is

$$
\nabla \cdot \boldsymbol{B}=0
$$

In the previous equations, $\boldsymbol{B}$ is the magnetic field, $c$ is the speed of light, $\boldsymbol{E}$ is the electric field, $n$ is the plasma density, $\boldsymbol{v}$ is the plasma bulk flow velocity, $m_{i}$ is the ion mass, $P$ is the pressure, and $\boldsymbol{J}$ is the current density.

With the six equations from ideal-MHD, we can derive general scaling laws for magnetic reconnection outside of the current layer. Inside the current layer, the ideal-MHD model breaks down, so additional terms in Ohm's law are needed to describe reconnection, as is discussed in Sections 1.2.1 and 1.2.3. For this exercise, we assume the plasma is incompressible (meaning the density $n$ does not change in the frame of the plasma), and the system is in the steady state (meaning $\partial / \partial t$ terms are 
equal to zero). We define $\delta$ to be the characteristic half-width of the reconnection current sheet, and $L$ as the characteristic half-length of the current sheet. The reconnection inflow velocities and outflow velocities are given as $v_{y}$ ( $\delta$ direction) and $v_{x}$ ( $L$ direction), respectively. We should note, the following scaling arguments are only valid for 2D magnetic reconnection. Three-dimensional reconnection will be discussed in Chapters 3 and 4 .

Consider the continuity equation (Eq. 1.3) in the steady state which gives $\nabla \cdot \boldsymbol{v}=0$. A scaling analysis yields $v_{x} / L \sim v_{y} / \delta$, or

$$
v_{y} \sim v_{x} \frac{\delta}{L}
$$

This equation gives us the scaling of the inflow velocity in terms of the parameters of the system. We perform a similar scaling analysis on Eq. 1.7, yielding $B_{x} / L \sim B_{y} / \delta$ or

$$
B_{y} \sim B_{x} \frac{\delta}{L}
$$

From the right-hand side of the momentum equation, Eq. 1.5 can be substituted into the $(\boldsymbol{J} \times \boldsymbol{B}) / c$ term can be re-written as

$$
\boldsymbol{J} \times \boldsymbol{B}=\frac{c}{4 \pi}\left[-\nabla \frac{B^{2}}{2}+(\boldsymbol{B} \cdot \boldsymbol{\nabla}) \boldsymbol{B}\right],
$$

where $\nabla B^{2} / 2$ is the magnetic pressure and $(\boldsymbol{B} \cdot \boldsymbol{\nabla}) \boldsymbol{B}$ is the tension (or curvature) force of the magnetic field. Plasma flows in the outflow are generated by the retreat of bent, reconnected magnetic fields from the X-line. If we balance the convection 
of the outflow $\left(\sim(\boldsymbol{v} \cdot \boldsymbol{\nabla}) v_{x}\right)$ with magnetic curvature we find that $m_{i} n v_{x}^{2} / L=$ $B_{y} B_{x} / 4 \pi \delta$. Using equations 1.8 and 1.9 , we find

$$
v_{x} \sim C_{A x}
$$

where $C_{A x}=B_{x} /\left(4 \pi m_{i} n\right)^{1 / 2}$ is the Alfvén speed due to the reconnecting $\left(B_{x}\right)$ magnetic field. Therefore, the plasma outflows due to magnetic reconnection are on the order of the Alfvén speed due to the reconnecting magnetic field $B_{x}$, which is expected if magnetic fields are controlling the dynamics.

Magnetic reconnection is often described in terms of its "reconnection rate", which is a measure of the amount of magnetic flux that is reconnected per unit time. In 2D, Faraday's law (Eq. 1.2) requires that the electric field be uniform in the steady state. The reconnection rate is proportional to the out-of-plane electric field $E_{z}$, which is shown by manipulating the integral form of Faraday's Law,

$$
\frac{d \Phi}{d t}=-c \oint_{C} \boldsymbol{E} \cdot d \boldsymbol{l}=-c E_{z} L_{Z}
$$

where $\Phi$ is the magnetic flux through the $x z$ plane that passes through the $\mathrm{X}$-line (the location of reconnection) and extends to infinity, $E_{z}$ is the out-of-plane electric field driving the out-of-plane current $J_{z}$, and $L_{Z}$ length in the out-of-plane direction. Upstream of the dissipation layer the electric field is given by Eq. 1.6, where ideal- 
MHD is valid. A scaling analysis reveals

$$
E_{z} \sim \frac{v_{y} B_{x}}{c}=\frac{v_{i n} B_{x}}{c}
$$

Therefore, $v_{i n}$ is a measure of the reconnection rate. Generally the reconnection rate $E^{\prime}$ is presented as a unitless quantity. This is achieved by normalizing $E_{z}$ with the upstream magnetic field strength $B_{x}$ and the outflow speed $v_{\text {out }}=v_{x}=C_{A x}$, therefore

$$
E^{\prime}=\frac{c E_{z}}{v_{\text {out }} B_{x}} \sim \frac{v_{\text {in }}}{v_{\text {out }}} \sim \frac{\delta}{L}
$$

\subsubsection{Sweet-Parker Reconnection}

We have developed general scaling laws for 2D steady magnetic reconnection. We can modify the ideal-MHD equations to consider a plasma system with finite resistivity $\eta$ (e.g., due to collisions between ions and electrons). Resistivity is introduced into ideal-MHD through Ohm's Law. We employ a uniform and constant resistivity, thus Ohm's Law becomes

$$
\boldsymbol{E}+\frac{\boldsymbol{v} \times \boldsymbol{B}}{c}=\eta \boldsymbol{J}
$$

Equation 1.15 replaces Eq. 1.6. Ideal-MHD with the resistive Ohm's Law is called resistive-MHD. If we substitute Eq. 1.15 into Eq. 1.2, we find

$$
\frac{\partial \boldsymbol{B}}{\partial t}=\nabla \times(\boldsymbol{v} \times \boldsymbol{B})+\frac{\eta c^{2}}{4 \pi} \nabla^{2} \boldsymbol{B}
$$


The $\nabla^{2} \boldsymbol{B}$ term is the rate of magnetic field diffusion within the dissipation region. The $\nabla \times(\boldsymbol{v} \times \boldsymbol{B})$ term breaks down into three terms,

$$
\boldsymbol{\nabla} \times(\boldsymbol{v} \times \boldsymbol{B})=(\boldsymbol{B} \cdot \boldsymbol{\nabla}) \boldsymbol{v}-(\boldsymbol{v} \cdot \boldsymbol{\nabla}) \boldsymbol{B}-\boldsymbol{B}(\boldsymbol{\nabla} \cdot \boldsymbol{v}) .
$$

The first term on the right hand side of the equation describes the bending of magnetic field lines. The second term describes the magnetic field lines convection in the direction of the bulk flow. The third term describes the compression of the magnetic fields, we assume the plasma is incompressible so $\boldsymbol{\nabla} \cdot \boldsymbol{v}=0$.

We can now re-write Eq. 1.16 as

$$
\frac{\partial \boldsymbol{B}}{\partial t}=(\boldsymbol{B} \cdot \boldsymbol{\nabla}) \boldsymbol{v}-(\boldsymbol{v} \cdot \boldsymbol{\nabla})+\frac{\eta c^{2}}{4 \pi} \nabla^{2} \boldsymbol{B},
$$

which describes the time evolution of the magnetic fields due to the bending, convection, and diffusion of the magnetic fields, respectively. If we consider the evolution of the reconnecting magnetic field $B_{x}$ in the steady state, upstream of the dissipation region, the plasma flow is purely in the inflow direction, so $v_{x} \sim 0$, therefore, Eq. 1.18 becomes

$$
0=-v_{y} \frac{\partial B_{x}}{\partial y}+\frac{\eta c^{2}}{4 \pi} \nabla^{2} B_{x} \rightarrow v_{y} \frac{\partial B_{x}}{\partial y}=\frac{\eta c^{2}}{4 \pi} \nabla^{2} B_{x} .
$$

Physically, this equation states that in the steady-state, the rate of the diffusion of magnetic fields in the dissipation region is equal to the rate at which magnetic field 
lines are convected into the dissipation region. A scaling analysis of Eq. 1.19 reveals

$$
\frac{v_{y}}{\delta} \sim \frac{\eta c^{2}}{4 \pi \delta^{2}}
$$

We can solve for the Sweet-Parker reconnection rate using Eq. 1.14 and 1.11. We can define the Sweet-Parker reconnection rate $E_{s p}^{\prime}$ as

$$
E_{s p}^{\prime} \sim \frac{v_{y}}{v_{x}} \sim \frac{\delta}{L} \sim\left(\frac{\eta c^{2}}{4 \pi c_{A x} L}\right)^{1 / 2} \sim S^{-1 / 2}
$$

where $S=4 \pi c_{A x} L / \eta c^{2}$ is the Lundquist number and the ratio of the Alfvén speed crossing time scale to the magnetic diffusion time scale. The Lundquist number is a dimensionless parameter and is characteristic of specific plasmas (i.e., $S \sim 10^{14}$ for plasmas in the solar corona). As stated earlier, the Sweet-Parker reconnection rate is not nearly fast enough ( $t \sim 4$ months) to explain observed reconnection rates. Eq. 1.21 shows that for systems where $L$ is particularly large (e.g., the corona) and the resistivity is particularly small (e.g., the corona), then the predicted reconnection rate due to Sweet-Parker reconnection is very slow.

\subsubsection{Sweet-Parker Enhanced by Secondary Islands}

A secondary tearing instability exists for systems of sufficient Lundquist number $\left(S_{\text {crit }} \geq 10^{4}\right.$ [Biskamp (1986)]). The secondary tearing instability spontaneously generates small magnetic islands, referred to as secondary islands, within the current sheet. Secondary islands enhance the Sweet-Parker reconnection rate because 


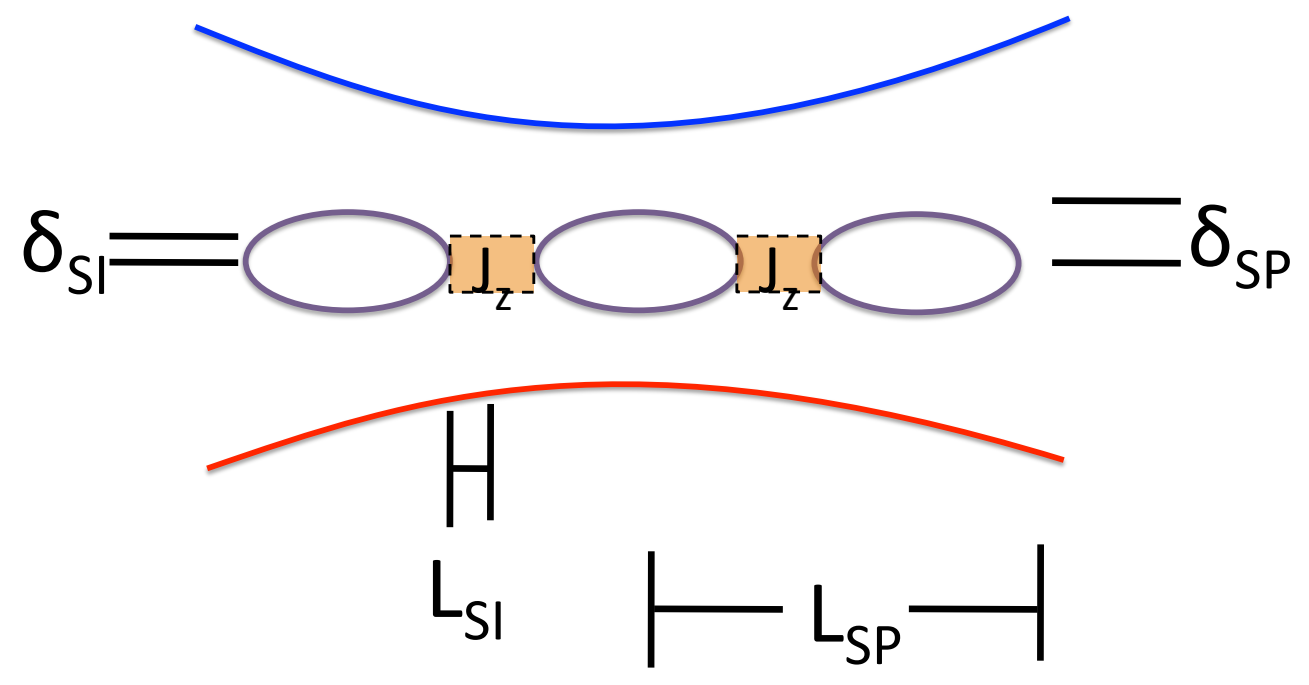

Figure 1.4: Sketch of secondary islands in a Sweet-Parker current sheet. The length of the current sheet decreases as secondary islands are generated, as the length of the current sheet decreases the half-width of the current sheet also decreases.

they allow for larger amounts of reconnected plasma to leave the current sheet in a shorter amount of time. Figure 1.4 details a Sweet-Parker current sheet broken up into multiple current sheets because multiple secondary islands (purple ovals) have formed.

How much faster is Sweet-Parker with secondary islands than the Sweet-Parker reconnection? Consider a current sheet of length $L$, where $N$ secondary islands spontaneously form. When $N$ secondary islands are formed, the current sheet fragments into $N$ individual current sheets with length $L_{S I}=L_{S P} / N$. If Sweet-Parker scaling holds for the new current sheets then

$$
E_{S I}=\left(\frac{\eta c^{2}}{4 \pi C_{A x} L_{S I}}\right)^{1 / 2}=N^{1 / 2} S^{-1 / 2} \Rightarrow \delta_{S I}=\frac{\delta_{S P}}{N^{1 / 2}} .
$$

The current sheets in secondary island reconnection are thinner than the current 
sheet during Sweet-Parker by a factor of $N^{-1 / 2}$ [Daughton et al. (2009b)]. Therefore, the Sweet-Parker reconnection rate due to the enhancement of secondary islands $E_{S I}$ is

$$
E_{S I}=\frac{\delta}{L}=E_{S P} N^{1 / 2}
$$

The reconnection rate due to the secondary island instability is faster than SweetParker reconnection. It is believed that secondary islands will be generated in each new current sheet until the local Lundquist number of an individual current sheet is less than the critical Lundquist number $\left(S_{\text {local }} \leq S_{\text {crit }}\right)$. Therefore, the number of islands generated for any current sheet scales like $N \sim S / S_{\text {crit }}$ [Cassak et al. (2009)]. The reconnection rate due to secondary islands then scales like

$$
E_{S I}=\left(\frac{S}{S_{\text {crit }}}\right)^{1 / 2} S^{-1 / 2}=S_{\text {crit }}^{-1 / 2}
$$

This argument predicts the reconnection rate due to secondary islands is weakly dependent on the Lundquist number [Cassak et al. (2009); Huang \& Bhattacharjee (2010); Uzdensky et al. (2010)] with a normalized characteristic reconnection rate $E_{S I} \sim 0.01$.

\subsubsection{Hall Reconnection}

In the quest to determine how energy is released so quickly during solar eruptions, it was discovered that the Hall effect allows for much faster reconnection rates fast enough to explain observations! The Hall effect appears in Ohm's law 
as $\boldsymbol{J} \times \boldsymbol{B} /$ nec. Again the only change required to introduce new physics into the ideal-MHD equations enters through Ohm's Law. Using the electron equation of motion, the modified Ohm's law can be derived as

$$
\boldsymbol{E}+\frac{\boldsymbol{v} \times \boldsymbol{B}}{c}=\eta \boldsymbol{J}+\frac{\boldsymbol{J} \times \boldsymbol{B}}{n e c}-\frac{\boldsymbol{\nabla} \cdot \boldsymbol{P}_{\boldsymbol{e}}}{n e}+\frac{m_{e}}{e^{2}} \frac{d \boldsymbol{J} / n}{d t} .
$$

This is called generalized Ohm's law [Rossi \& Olbert (1970)], where the three additional terms added to resistive-MHD are the Hall term, electron pressure gradient term, and electron inertia term, respectively. The resistivity and off-diagonal electron pressure terms can break the frozen-in condition, which allows for a change in the magnetic topology because they are dissipative. In this thesis, the off diagonal terms are equal to zero because the contribution is only important at electron scales and the off diagonal terms only serve to change the magnetic topology. The pressure gradient term becomes $\nabla p_{e} / n e$. We assume $p_{e}=n T_{e}$ with a uniform and constant electron temperature.

However, the Hall term does not allow the magnetic topology to change, but it does have a significant effect on the reconnection rate. The Hall term only contributes to magnetic reconnection if the current sheet is on the order of the ion inertial scale $d_{i}$, also known as the ion skin depth. We define the ion gyroradius to be $d_{i}=c_{A} / \Omega_{c i}=c / \omega_{p i}=\left(m_{i} c^{2} / 4 \pi n e^{2}\right)^{1 / 2}$, where $\Omega_{c i}$ is the ion cyclotron frequency and $\omega_{p i}$ is the plasma frequency, $c$ is the speed of light, and $c_{A}=B^{2} /\left(4 \pi m_{i} n\right)^{1 / 2}$ is the Alfvén speed. The Hall term allows ions to decouple from magnetic field lines and de-magnetize. According to the frozen-in law, electrons and ions orbit around 
the same magnetic field line, indefinitely, but if an ion (or electron) gyrating around a field line encounters a magnetic field line that points in the opposite direction within a gyro-orbit then the ion begins to orbit in the opposite direction, losing its connection with the first field line [Speiser (1970)]. This motion is called meandering. Electrons can also exhibit meandering. This occurs at the electron gyro radius (or electron skin depth) $d_{e}=c / \omega_{p e}=\left(m_{e} c^{2} / 4 \pi n e^{2}\right)^{1 / 2}$, where $\omega_{p e}$ is the electron plasma frequency and $m_{e}$ is the electron mass. The ion skin depth is much larger than the electron skin depth because $m_{i}>>m_{e}$.

The Hall term introduces new physics into the magnetic reconnection picture and exhibits a unique reconnection signature when the current layer is below the ion gyro-radius. At length scales smaller than the ion gyroradius the ion bulk flow is near zero, therefore the current is carried primarily by the electron flow [from $\left.\boldsymbol{J}=n e\left(-\boldsymbol{v}_{e}+\boldsymbol{v}_{i}\right)\right]$. This current, created by the electron flow, is called the Hall current and flows the opposite direction of the electron current in the downstream direction. The Hall current is visualized in Fig. 1.5 by the dashed blue line. From the right hand rule, this current creates a quadrupole magnetic field $\left(B_{z}\right)$ in the outof-plane direction. The quadrupole structure has been shown in Sonnerup (1979). The Hall term was shown to be related to the physics of whistler waves [Mandt et al. (1994)]. Hall reconnection has been observed in the magnetosphere [Øieroset et al. (2001); Mozer et al. (2002); Scudder et al. (2002); Runov et al. (2003)] and laboratory experiments [Ren et al. (2005); Cothran et al. (2005)].

How does the Hall term allow for fast reconnection rates? The Hall term is responsible for the opening of the current layer into an open Petschek-like config- 


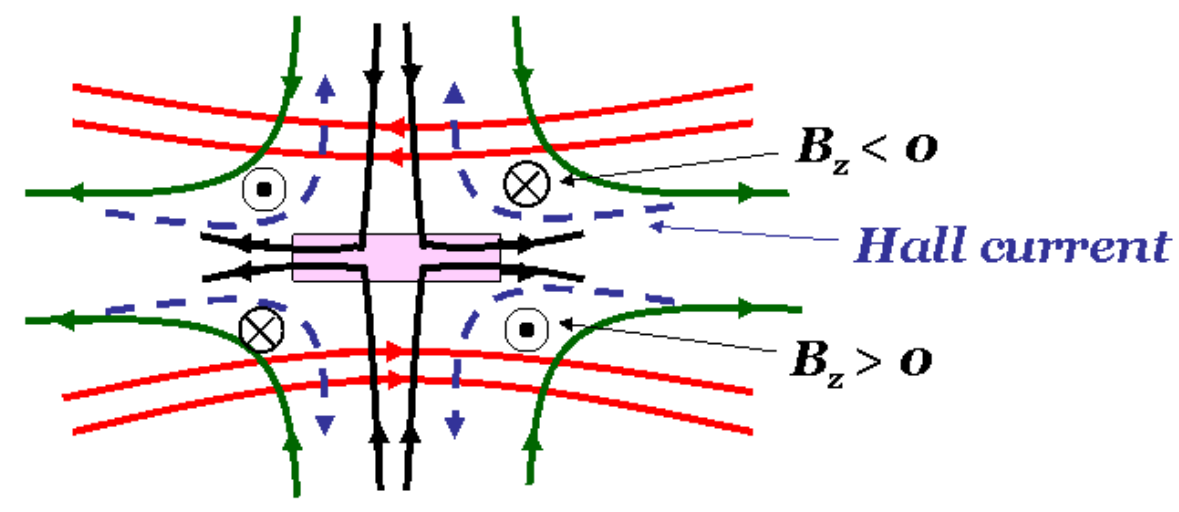

Figure 1.5: The quadropolar out-of-plane magnetic field structure during Hall reconnection. The Hall current (blue-dashed lines) flows along the inflow direction opposite the electron bulk flow from the still magnetized electrons. This current generates an out-of-plane magnetic field. Reprinted with permission from Ref. [Cassak (2006)].

uration by introducing the physics of whistler waves, or kinetic Alfvén waves in the presence of a guide field (the $B_{z}$ component of the magnetic field). The role of whistler waves and kinetic Alfvén waves in the appearance of fast magnetic reconnection is somewhat controversial. In Sweet-Parker reconnection the outflow is controlled by the curvature of magnetic field, i.e., Alfvén wave physics with wave speed $\omega / k=c_{A}$. The Alfvén wave is non-dispersive (wave speed does not depend on $k$ ), so the outflow is constant regardless of length scales. The whistler wave is a dispersive wave, because the wave speed $\omega / k \sim k c_{A} d_{i}$ depends on $k$, so the flow speed depends on the distance from the center of the reconnection exhaust.

Why is the dispersive nature of the wave important? For non-dispersive waves the outflow speed does not change along the inflow direction. The reconnection exhaust remains collapsed due to the continuity equation Eq. 1.3. In the steady 
state, we find

$$
\frac{\partial \rho v_{x}}{\partial x} \sim-\frac{\partial \rho v_{y}}{\partial y}
$$

If the exhaust opens even slightly the mass flux along the outflow direction increases, i.e., $\partial\left(\rho v_{x}\right) / \partial x>0$. To preserve the mass flux an inflow towards the center of the exhaust $\left(v_{y}<0\right)$ is required that will pull the magnetic field towards the center of the exhaust, closing the exhaust into a long, thin current sheet reminiscent of Sweet-Parker reconnection. With dispersive waves, the wave speed decreases along the inflow direction away from the center of the exhaust, i.e., $\partial\left(\rho v_{x}\right) / \partial x<0$. To preserve continuity, a vertical flow away from the center of the exhaust is required $\left(v_{y}>0\right)$. This flow drags the magnetic field away from the center of the exhaust into the open X-type configuration.

In Sweet-Parker reconnection, we develop scaling arguments from resistiveMHD and determine reconnection rates, current sheet parameters, and so on. Unfortunately, Hall reconnection has no such scaling arguments! Then how fast is Hall reconnection? Large scale numerical simulations show that the reconnection rate $E_{\text {Hall }} \sim 0.1$ and is independent of system size [Shay et al. (1999); Birn et al. (2001); Huba \& Rudakov (2004)]. Hall reconnection has been observed experimentally [Ren et al. (2005); Cothran et al. (2005)] with reconnection rates faster than Sweet-Parker reconnection rates. 


\subsubsection{Anomalous Resistivity}

Anomalous resistivity also leads to reconnection rates on the order of Hall reconnection [Sato \& Hayashi (1979); Biskamp (1986); Scholer (1989)]. Sweet-Parker reconnection employs a constant and uniform resistivity. Anomalous resistivity can be constant over time, but not spatially uniform. What does this mean for reconnection dynamics? In panel (a) of Fig. 1.6, we consider magnetic reconnection with a constant and uniform resistivity. The magnetic fields convect towards the neutral line and annihilate due to the resistivity. Due to uniform resistivity, the magnetic fields move uniformly and create a long, thin Sweet-Parker current sheet. In panel (b), the resistivity is localized in the region of the out-of-plane current $J_{z}$ and falls off quickly to zero as we move horizontally from the X-line. Since the resistivity is not uniform, the magnetic fields will not uniformly move towards the neutral line. Therefore, only a small section of the magnetic fields are actually annihilated and an open reconnection outflow is formed with a short, thin current sheet. Simulations have shown that the reconnection rate is also fast $E_{\text {anom }} \sim 0.1$. It has been shown that instabilities that onset on small length scales can locally enhance the resistivity. The lower hybrid drift instability [Huba et al. (1977)] and the Buneman instability [Drake et al. (2003)] may cause anomalous resistivity in the earth's magnetotail.

\subsection{MHD Discontinuities}

MHD discontinuities play a significant role in magnetic reconnection. Analysis of discontinuities is important to explain observations and the physics of reconnec- 


\section{a) Uniform Resistivity}

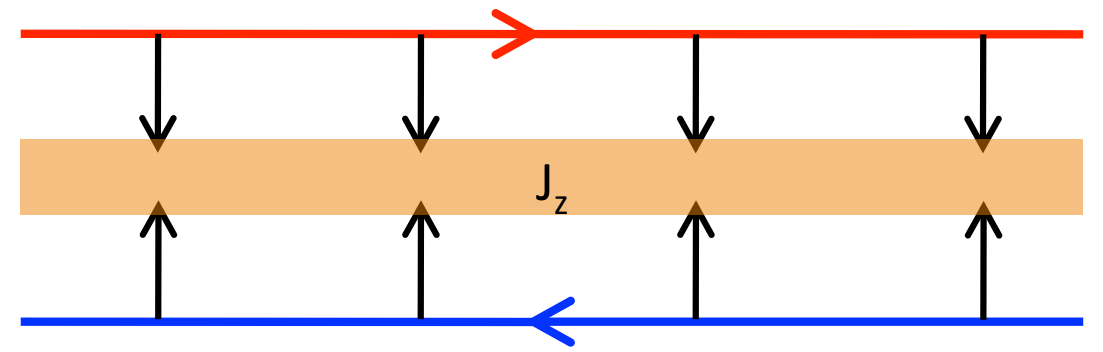

b) Anomalous Resistivity

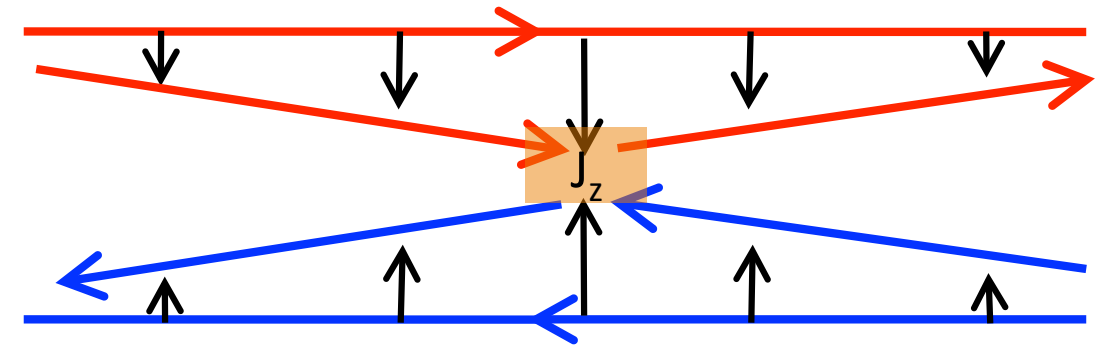

Figure 1.6: (a) Diagram of the evolution of the magnetic fields for a constant and uniform resistivity. The magnetic fields annihilate evenly and pull large sections of the magnetic field towards the middle of the current sheet. (b) Diagram of the evolution of the magnetic fields for an anomalous resistivity. The magnetic fields annihilate only a small section of the incoming magnetic fields creating an open X-type configuration. Adapted from Ref. [Kulsrud (2001)]. 
tion. An MHD discontinuity is a thin layer between two populations of plasma through which the pressure, flow velocity, magnetic field, and plasma density may change significantly. Discontinuities form in the reconnection outflows [Petschek (1964); Levy et al. (1964); Vasyliunas (1975); Shi \& Lee (1990); Lin \& Lee (1993)] as a by-product of the reconnection process. As we cross between boundary layers, physical quantities can vary greatly. In MHD four types of discontinuities exist: contact discontinuity, tangential discontinuity, rotational discontinuity, and MHD shocks [Landau \& Lifshitz (1960)]. We analyze MHD discontinuities using the conservative form of the ideal-MHD equations:

$$
\begin{gathered}
\frac{\partial \rho}{\partial t}+\nabla \cdot(\boldsymbol{v} \rho)=0 \\
\frac{\partial(\rho \boldsymbol{v})}{\partial t}+\nabla \cdot\left[\rho \boldsymbol{v} \boldsymbol{v}+\left(P+\frac{B^{2}}{8 \pi}\right) \boldsymbol{I}-\frac{\boldsymbol{B} \boldsymbol{B}}{4 \pi}\right]=0 \\
\frac{\partial}{\partial t}\left[\frac{1}{2} \rho v^{2}+\frac{P}{\gamma-1}+\frac{B^{2}}{8 \pi}\right]+\nabla \cdot\left[\left(\frac{1}{2} \rho v^{2}+\frac{P}{\gamma-1}+\frac{B^{2}}{8 \pi}\right) \boldsymbol{v}-\frac{1}{4 \pi}(\boldsymbol{v} \cdot \boldsymbol{B}) \boldsymbol{B}\right]=0 \\
\frac{\partial \boldsymbol{B}}{\partial t}=-c \boldsymbol{\nabla} \times \boldsymbol{E} \\
\boldsymbol{\nabla} \cdot \boldsymbol{B}=0
\end{gathered}
$$

where $\gamma$ is the ratio of specific heats. Assuming a steady state and integrating each equation over a Gaussian pillbox across a discontinuity, we find the RankineHugoniot (RH) jump conditions:

$$
\left[\rho v_{n}\right]=0
$$




$$
\begin{gathered}
{\left[\rho v_{n}^{2}+\left(P+\frac{B^{2}}{8 \pi}\right)-\frac{B_{n}^{2}}{4 \pi}\right]=0} \\
{\left[\rho v_{n} \boldsymbol{v}_{\boldsymbol{t}}-\frac{B_{n} \boldsymbol{B}_{\boldsymbol{t}}}{4 \pi}\right]=0} \\
{\left[\left(\frac{1}{2} \rho v^{2}+\frac{P}{\gamma-1}+\frac{B^{2}}{4 \pi}\right) v_{n}-\frac{\boldsymbol{v} \cdot \boldsymbol{B}}{4 \pi} B_{n}\right]=0} \\
{\left[B_{n} \boldsymbol{v}_{\boldsymbol{t}}-v_{n} \boldsymbol{B}_{\boldsymbol{t}}\right]=0} \\
{\left[B_{n}\right]=0,}
\end{gathered}
$$

where the $n$ and $t$ subscripts represent the normal and tangential component of the quantity, respectively. The square brackets represent the difference between the quantities on either side of the discontinuity. For example, $[L]=L_{2}-L_{1}$ where the subscript ' 1 ' represents the values upstream of the discontinuity and '2' denotes the values downstream of the discontinuity. The RH jump conditions describe the conservation of mass, momentum, energy, normal magnetic field $\left(B_{n}\right)$, and tangential electric field $\left(E_{t}\right)$. The RH jump relations for each MHD discontinuity are discussed below.

\subsubsection{Contact Discontinuity (CD)}

A CD occurs when $v_{n}=0$, then from Eq. 1.34 we find that $\left[\boldsymbol{B}_{\boldsymbol{t}}\right]=0$. Equation 1.36 shows that $\left[v_{t}\right]=0$. Eq. 1.33 shows the plasma pressure is the same on either side $([P]=0)$. However, the temperature and density need not be conserved across the CD. Therefore, a CD is the discontinuity between two plasma populations with different temperatures and densities. The CD jump conditions are summarized 

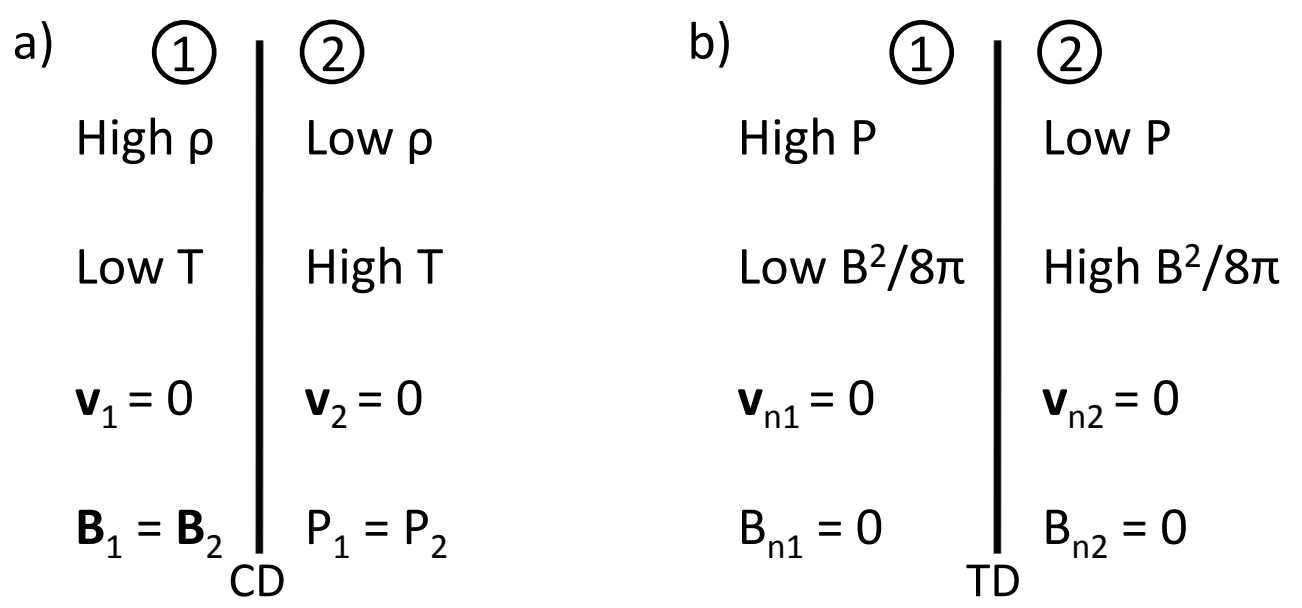

Figure 1.7: (a) Diagram of a contact discontinuity (CD). (b) Diagram of a tangential discontinuity (TD).

in Fig. 1.7(a).

\subsubsection{Tangential Discontinuity (TD)}

We now consider when $v_{n}=0$ and $B_{n}=0$. From Eq. 1.33, the jump condition becomes $\left[P+B^{2} / 8 \pi\right]=0$. The TD separates two plasmas with different magnetic fields and plasma pressures. Also, $\left[B_{t}\right] \neq 0$ because the normal components of the magnetic field and flow velocity are equal to zero. The TD jump conditions are summarized in Fig. 1.7(b). The current layer before magnetic reconnection begins is a tangential discontinuity.

\subsubsection{Rotational Discontinuity (RD)}

We consider a system where the jump conditions $[P]=0$ and $[\rho]=0$. Using Eq. 1.34 and Eq. 1.36, we find that $v_{n}=B_{n} /(4 \pi \rho)^{1 / 2}=C_{A n}$. The flow normal to 
the discontinuity is equal to the Alfvén velocity due to the normal component of the magnetic field. Substituting $v_{n}$ into Eq. 1.36 yields the jump condition for the tangential velocity $\left[v_{t}\right]=\left[B_{t}\right] /(4 \pi \rho)^{1 / 2}$. This is called the Walen relation for the RD, which is useful for identifying rotational discontinuities in observations. The jump conditions for RDs are summarized in Fig. 1.8(a) and (b), where the blue arrow represents the magnetic field and the red arrow represents the plasma flow normal to the discontinuity. RDs in essence "rotate" the plasma flow and magnetic field away from their original orientation.

\subsubsection{MHD Shocks}

MHD shocks are related to propagating wave modes and are formed when non-linear waves steepen. We find the properties for MHD shocks, assuming that the plasma flow $v_{1}=v_{1 n}$ is such that the tangential velocity is zero $\left(v_{t 1}=0\right)$. The magnetic field $B_{1}$ makes an angle of $\theta$ with the direction normal to the discontinuity. This configuration is shown in Fig. 1.8(a). The jump conditions Eq. 1.32, 1.36, and 1.34 then become:

$$
\begin{gathered}
\frac{v_{2 n}}{v_{1 n}}=\frac{\rho_{1}}{\rho_{2}} \\
B_{n} \boldsymbol{v}_{2 t}=v_{2 n} \boldsymbol{B}_{2 t}-v_{1 n} \boldsymbol{B}_{1 t} \\
\rho_{1} v_{n 1} \boldsymbol{v}_{2 t}=\frac{B_{n}}{4 \pi}\left(\boldsymbol{B}_{2 \boldsymbol{t}}-\boldsymbol{B}_{1 \boldsymbol{t}}\right) .
\end{gathered}
$$


Solving equations $1.38,1.39$ and 1.40 for the shocked tangential magnetic field $\boldsymbol{B}_{2 t}$ and and the shocked tangential plasma flow $\boldsymbol{v}_{2 t}$, we find

$$
\begin{gathered}
\boldsymbol{B}_{2 t}=\frac{M_{A 1}^{2}-\cos ^{2} \theta}{M_{A 1}-r \cos ^{2} \theta} r \boldsymbol{B}_{1 t} \\
\boldsymbol{v}_{2 t}=\frac{v_{1 n}}{B_{n}}\left(\frac{(r-1) \cos ^{2} \theta}{M_{A 1}^{2}-r \cos ^{2} \theta}\right) \boldsymbol{B}_{1 t},
\end{gathered}
$$

where $r=\rho_{2} / \rho_{1}$ is the compression ratio and $M_{A 1}=v_{n 1} / c_{A 1}$ is the Alfvén Mach number. Substituting these two equations into Eq.1.40, we find

$$
\left(\frac{a M_{a 1}^{2}}{r}-b\right)\left(\frac{M_{A 1}^{2}}{r}-\cos ^{2} \theta\right)^{2}-\frac{M_{A 1}^{2}}{r} \sin ^{2} \theta\left[\frac{M_{A 1}^{2}}{r}\left(\frac{a}{r}-\frac{1-r}{2}\right)-a \cos ^{2} \theta\right]=0
$$

where $a=[(\gamma+1)-(\gamma-1) r] / 2$ and $b=\beta_{1} / 2$. The term $\gamma$ is the ratio of specific heats and is typically valued $\gamma=5 / 3$. The ratio of gas pressure and magnetic pressure is defined as the plasma beta $\left(\beta_{1}=P_{1} /\left(B_{1}^{2} / 4 \pi\right)\right)$.

In the $M_{A 1} \rightarrow \infty$ limit, the only solution to Eq.1.43 is when $a \sim 1 / M_{A 1}^{2}$. Substituting in the value for $a$, we find that $r=(\gamma+1) /(\gamma-1)=4$, given that $\gamma=5 / 3$. Then by substituting into Eq. 1.41, we find the shocked tangential magnetic field $\boldsymbol{B}_{2 t}=4 \boldsymbol{B}_{1 t}$, and the normal velocity of the plasma flow $v_{2 n}=v_{1 n} / 4$. The density of the plasma $\rho_{2}=4 \rho_{1}$ also increases significantly. This result indicates that kinetic energy is converted to gas and magnetic pressure. The magnetic field and gas pressure increase in parallel and is attributed to the physics of a fast magnetosonic wave. This result is displayed in a sketch of the shock in Fig. 1.8(c). This type of shock is called a fast shock or "switch-on" shock, because the magnetic field's 

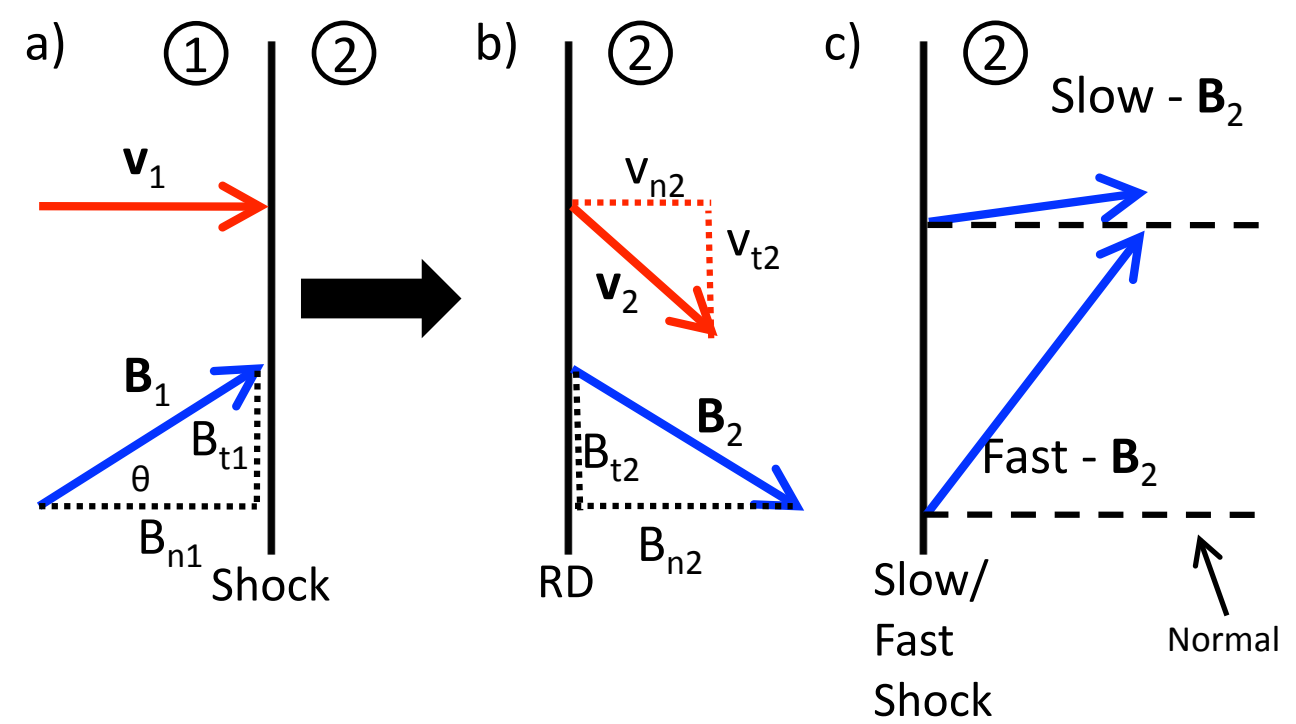

Figure 1.8: (a) Initial magnetic field and plasma flow parameters. (b) Diagram of a rotational discontinuity (RD). (c) Diagram of a slow ("switch-off") and fast shock ("switch-on").

tangential component is bent away from the normal direction.

In the $M_{A 1} \rightarrow 0$ limit, Eq. 1.43 becomes $M_{A 1}^{2} \simeq r b \cos ^{2}(\theta) / a$. Substituting into Eq. 1.41, we find that $B_{1 t}>B_{2 t}$, the tangential magnetic field is decreased across the shock. The compression ratio $r>1$ for this shock so $v_{2 n}$ decreases and $\rho_{2}$ increases. Therefore, the kinetic and magnetic energy are converted into thermal energy. This type of shock is called a slow shock or "switch-off" shock, because the magnetic field is bent towards the the normal. This result is summarized in Fig. 1.8.

\subsection{Summary of Research}

In this thesis, we present three studies of magnetic reconnection by employing two-dimensional and three-dimensional large-scale numerical simulations. Chapter 2 addresses the role of secondary islands in the release of energy in solar flare 
events. Secondary islands make Sweet-Parker faster [Matthaeus \& Lamkin (1985); Kliem (1995); Lazarian \& Vishniac (1999); Lapenta (2008); Huang \& Bhattacharjee (2010)]. Can secondary islands make Sweet-Parker reconnection fast enough? It is believed by some that Sweet-Parker with secondary islands can increase the reconnection rate sufficiently to explain observations. In this study, we present the first simulation to separate the three regimes of reconnection, Sweet-Parker, Sweet-Parker with secondary islands, and Hall reconnection, where each form of reconnection occurs in its own distinct phase, without effects from the other regimes of reconnection playing a role in the dynamics. This study had three results that contribute to the energy release picture: 1) there exists a regime in which secondary islands occur without collisionless (Hall) effects playing a significant role; (2) the reconnection rate due to secondary islands is faster than Sweet-Parker but still significantly slower than Hall reconnection, which shows secondary islands are not the cause of the highest reconnection rates; and (3) the onset of Hall reconnection ejects secondary islands in the vicinity of the X-line. Results 2 and 3 imply that energy release is the most efficient during Hall reconnection [Shepherd \& Cassak (2010)].

Chapter 2 addresses magnetic reconnection in 2D. This is an idealized case, as most instances in nature, magnetic reconnection is inherently a three-dimensional process. Naturally occurring magnetic reconnection often begins in a localized region and spreads in the out-of-plane direction (perpendicular to the plane of reconnection). As reconnection spreads, new regions of reconnection are triggered because the reconnection signal propagates in the out-of-plane direction by two possible mechanisms. When the guide field is small compared to the reconnecting field, re- 
connection spreads in the out-of-plane direction by the current carriers (electrons). Current carrying spreading has been studied with large scale computer simulations with a focus on magnetotail applications [Huba \& Rudakov (2002, 2003); Shay et al. (2003); Karimabadi et al. (2004); Lapenta et al. (2006); Lukin \& Linton (2011); Nakamura et al. (2012)] that favor a weak guide field. Systems with large guide fields exhibit much different spreading behavior. It was observed in experiments performed at the Versatile Toroidal Facility (VTF) that magnetic reconnection begins in a localized region and spreads bi-directionally in the out-of-plane direction with speeds consistent with the Alfvén speed due to the guide field. Bi-directional spreading was also observed in two-ribbon flares [Qiu (2009); Qiu et al. (2010)], where the guide field was expected to be sizeable compared to the reconnecting magnetic field. The spreading mechanism appears strongly dependent on the guide field. In this study, we confirm this is the case with numerical simulations and predict the critical guide field at which the spreading mechanism changes from current carrying spreading to Alfvén wave spreading. The prediction is tested with a series of simulations varying the guide magnetic field. It was found that magnetic reconnection spreads due to current carriers for $B_{g} \leq 2.0$, and Alfvén wave spreading occurs when $B_{g}>2.0$ [Shepherd \& Cassak (2012)].

In the solar wind, magnetic reconnection exhausts with widths approximately 390 Earth radii $\left(R_{E}\right)$ have been observed [Phan et al. (2006)]. What is the cause of the large scale reconnection structure? Phan et al., 2006 hypothesized the reconnection begins in a small region near the sun and gets larger over time as it convects with the solar wind. Another possibility is that the reconnection is localized (fixed) in 
the out-of-plane direction, where the reconnection exhaust can expand into the outof-plane direction, thus accounting for the observed reconnection site. We address this problem in Chapter 4 . We discuss the structure of the three-dimensional reconnection exhaust when reconnection remains localized in the out-of-plane direction. Magnetic reconnection remains localized by employing anomalous resistivity that is localized in the out-of-plane direction. It was found that in the presence of a guide field, the reconnection exhaust (therefore, the reconnection signatures) propagate out beyond the region of localized reconnection, where the exhaust forms a ribbon structure and the extent to which the reconnection expands was strongly dependent on the guide field strength. It was also found that the exhaust is bounded by four MHD discontinuities. Two of the boundaries of the exhaust were rotational discontinuities, where plasma flows across the discontinuity. The other two boundaries were tangential discontinuities, where there is no flow normal to the discontinuity. This result is then used to assess the large exhaust events in the solar wind, and an application to observations of solar flares is discussed. 


\section{Chapter 2}

\section{The transition between Sweet-Parker with secondary islands to Hall}

\section{reconnection}

As discussed in Chapter 1, magnetic reconnection is a mechanism that releases stored energy in magnetic fields. In the corona, solar flares release massive amounts of energy into interplanetary space. Solar flare events have been observed and studied for over 150 years, but there is still debate on the role of magnetic reconnection in flare dynamics. Particularly, debate surrounds how energy is released so fast. Two phases of reconnection discussed in the previous chapter may explain this rapid energy release. Sweet-Parker reconnection enhanced by secondary islands and Hall reconnection both exhibit faster reconnection rates than the slow Sweet-Parker reconnection prediction, but are they fast enough to explain energy release rates and can the parameter regimes they require be met in the solar corona? This poses an important question: when both phases of reconnection can be realized which phase of reconnection mediates the energy release?

This chapter will shed light on the transition of reconnection from slow to fast reconnection with a focus on coronal applications. In Section 2.1, we briefly discuss previous work on the effect of secondary islands and the Hall effect on the reconnection process. In Section 2.2, the first simulation to separate the effects of secondary islands and the Hall effect is discussed, along with the simulation nu- 
merics, relevant scaling analysis, and predictions. In Section 2.3, the results of the study are discussed, where there are three main results: (1) there is a regime in which secondary islands occur without collisionless effects playing a role; (2) the reconnection rate due to secondary islands is faster than Sweet-Parker reconnection but significantly slower than Hall reconnection, which shows that secondary islands are not the cause of the highest reconnection rates; and (3) the onset of Hall reconnection ejects secondary islands in the vicinity of the X-line. The implications of these results are then discussed in Section 2.4. All of the results presented in this chapter were published in Physical Review Letters in July 2010 [Shepherd \& Cassak (2010)]

\subsection{Introduction}

The first self-consistent theory to explain energy release in the solar flares was the Sweet-Parker model [Sweet (1958); Parker (1957)], but is far too slow to explain observations. Much has been invested in faster reconnection scenarios, such as collisionless (Hall) reconnection [Birn et al. (2001)] in which the Hall term plays a key role [Mandt et al. (1994); Rogers et al. (2001); Malakit et al. (2009); Cassak et al. (2010)]. Hall reconnection seems fast enough to explain observed energy release rates [Shay et al. (1999)]. Lately, the role of secondary islands (plasmoids) on Sweet-Parker reconnection has generated much interest. While they were discussed some time ago [Matthaeus \& Lamkin (1985, 1986); Biskamp (1986)], systematic studies were not carried out until recently. It has been argued in various contexts 
that secondary islands make reconnection faster than Sweet-Parker reconnection [Matthaeus \& Lamkin (1985); Kliem (1995); Lazarian \& Vishniac (1999); Lapenta (2008); Huang \& Bhattacharjee (2010)]. (Note, we are discussing secondary islands occurring during collisional reconnection, not those that occur after collisionless reconnection has begun [Daughton et al. (2006); Fermo et al. (2012)].)

Understanding secondary islands in Sweet-Parker reconnection is important for explaining coronal evolution. On the theoretical side, the reconnection rate places constraints on the dynamics. For example, if secondary islands make Sweet-Parker reconnection much faster or hasten the transition to fast reconnection, it cannot take place during pre-flare energy storage. If it remains slow, then it can occur while energy accumulates [Cassak et al. (2005); Uzdensky (2007); Cassak et al. (2008)]. On the observational side, it was hypothesized that high density blobs in current sheets during solar eruptions are secondary islands [Ciaravella \& Raymond (2008); Lin et al. (2009)]. Also, numerous observations of reconnection processes display a slow phase preceding an eruptive event with an abrupt transition. Examples include noneruptive flux emergence [Longcope et al. (2005)] and flows during coronal implosions as a result of an impulsive flare [Liu et al. (2009); Liu \& Wang (2010)].

Past theoretical work on secondary islands showed they appear spontaneously due to a secondary tearing instability when the Lundquist number $S=4 \pi c_{A} L_{S P} / \eta c^{2}$ exceeds $\sim 10^{4}$ [Biskamp (1986)], where $L_{S P}$ is the half-length of the Sweet-Parker dissipation region, $\eta$ is the resistivity, and $c_{A}$ is the Alfvén speed based on the reconnecting magnetic field. Equivalently, this can be written as $\delta / L_{S P}<0.01$, where $\delta$ is the thickness of the dissipation region. A study of the linear phase of the 
instability [Loureiro et al. (2007)] found a growth rate faster than the Alfvén transit time along the sheet. Recent simulations addressed the nonlinear reconnection rate $E$ for high $S$, showing it is considerably faster than the Sweet-Parker rate and its dependence on $S$ becomes weak [Bhattacharjee et al. (2009); Cassak et al. (2009); Loureiro et al. (2012)]. However, the simulations only go up to $S \sim 10^{7}$, so $E$ is only one order of magnitude faster than the Sweet-Parker rate and it is not clear whether it will be fast or slow at larger $S$. Other relevant studies showed that $E$ increases with the square root of the number of islands [Daughton et al. (2009b); Cassak et al. (2009)] and secondary islands are suppressed when reconnection is embedded, meaning the upstream field is smaller than the asymptotic field [Cassak \& Drake (2009)]. Many studies consider secondary islands caused by external random magnetic perturbations [Smith et al. (2004); Kowal et al. (2009); Loureiro et al. (2009); Skender \& Lapenta (2010)]. Other studies include the interaction of multiple islands [Nakamura et al. (2010)] and a statistical model of multiple island interaction [Fermo et al. (2010)]. A recent study has probed the effect of secondary islands in the Hall dominated regime, where Hall reconnection current sheets are shown as unstable and collapsing back into collisional current layers in certain parameter regimes [Huang et al. (2011)].

In addition to increasing the reconnection rate, secondary islands hasten the transition to Hall reconnection [Shibata \& Tanuma (2001); Daughton et al. (2009b); Huang et al. (2011); Baalrud et al. (2011)]. When a secondary island forms, the fragmented current sheet is shorter, so its Sweet-Parker thickness is smaller [Daughton et al. (2009b); Cassak et al. (2009)]. When the layer reaches ion gyroscales [Mandt 
et al. (1994); Ma \& Bhattacharjee (1996)], Hall reconnection begins abruptly [Bhattacharjee (2004); Cassak et al. (2005, 2006, 2007); Daughton et al. (2009b)]. This was recently verified using collisional particle-in-cell (PIC) simulations [Daughton et al. $(2009 b, a)]$.

The only previous study to include both secondary islands and the Hall effect utilized large PIC simulations [Daughton et al. (2009b,a)], but numerical constraints forced $S$ to be small enough that Hall reconnection began as soon as a secondary island formed. Since the Hall effect arises only at ion gyroscales, there should be a regime in which secondary islands are present without the Hall effect playing a role, if the sheet is thicker than ion gyroscales. The goal of this study is to separate the two effects and ascertain which one leads to dramatically larger reconnection rates, and which dictates the mechanism releasing the majority of the energy during the eruptive phase of reconnection.

\subsection{Simulation Setup}

Numerical simulations are performed using the two-fluid code F3D [Shay et al. (2004)]. Magnetic fields and densities are normalized to arbitrary values $B_{0}$ and $n_{0}$,

velocities to the Alfvén speed $c_{A 0}=B_{0} /\left(4 \pi m_{i} n_{0}\right)^{1 / 2}$ where $m_{i}$ is the ion mass, lengths to the ion inertial length $d_{i 0}=c / \omega_{p i}$, times to the ion cyclotron time $\Omega_{c i}^{-1}$, electric fields to $E_{0}=c_{A 0} B_{0} / c$, and resistivity to $\eta_{0}=4 \pi c_{A 0} d_{i 0} / c^{2}$.

The initial configuration is a double tearing mode with two Harris sheets, $B_{x}(y)=\tanh \left[\left(y+L_{y} / 4\right) / w_{0}\right]-\tanh \left[\left(y-L_{y} / 4\right) / w_{0}\right]-1$, where $w_{0}$ is the initial 
current layer thickness and $L_{y}$ is the system size in the inflow direction. Total pressure is balanced initially using a non-uniform density which asymptotes to 1 . The temperature $T=1$ is constant and uniform. A single X-line is seeded using a coherent magnetic perturbation of amplitude $1.6 \times 10^{-2}$ to rapidly achieve nonlinear reconnection. Initial random magnetic perturbations break symmetry so secondary islands are ejected. There is no initial out-of-plane (guide) magnetic field. Boundaries in both directions are periodic. Electron inertia is $m_{e}=m_{i} / 25$. This value is acceptable since we focus on the onset of Hall reconnection at ion scales rather than electron scales.

Simulation parameters are chosen so reconnection will proceed in three distinct phases: Sweet-Parker without secondary islands, Sweet-Parker with secondary islands, and Hall reconnection. A very large system size $L_{x} \times L_{y}=819.2 \times 409.6$ is employed with resistivity $\eta=0.008$, corresponding to a global Lundquist number $S_{g}=L_{x} / \eta \sim 10^{5}$, which exceeds the Biskamp criterion of $10^{4}$. To postpone secondary island onset, we choose $w_{0}=12.0$ which makes the reconnection embedded [Cassak \& Drake (2009)]. Embedding makes the Sweet-Parker layer thicker since $\delta \sim\left(\eta L_{S P} / c_{A u p}\right)^{1 / 2}$, where $c_{A u p}$ is the Alfvén speed based on the upstream magnetic field $B_{u p}$. For wide current layers, $B_{u p} \sim B_{0} \delta / w_{0}$ [Cassak \& Drake (2009)], so eliminating $B_{u p}$ gives $\delta \sim\left(\eta L_{S P} w_{0}\right)^{1 / 3} \sim 2.7$, where $L_{S P} \sim L_{x} / 4 \sim 200$ in our periodic system. Thus, the layer begins wider than $d_{i}$, and since $\delta / L_{S P}>0.01$, no secondary islands occur initially and the system undergoes Sweet-Parker reconnection. The reconnection inflow convects in stronger magnetic fields, so the current sheet self-consistently thins. Islands arise when $\delta / L_{S P} \sim 0.01$, which gives $\delta \sim 2.0$ since 
$L_{S P} \sim 200$. It has been shown [Daughton et al. (2009b); Cassak et al. (2009)] that if $N$ X-lines are present, $\delta$ decreases by a factor of $N^{1 / 2}$. For a single secondary island $(N=2)$, the layer shrinks to $\delta \sim 2.0 / 2^{1 / 2} \sim 1.4$. This exceeds $d_{i}$, so Sweet-Parker with secondary islands should persist. Hall reconnection only starts when $\delta \sim 1$, so three distinct phases occur.

A simulation is first performed with a grid scale $\Delta=0.2$ and the results are qualitatively consistent with expectations. To assure $\Delta$ does not play a role, the simulations are redone with $\Delta=0.1$, giving comparable results. Data is presented only from the high resolution runs. The equations employ fourth order diffusion (with form $D_{4} \nabla^{4}$ ) with coefficient $D_{4}=1.75 \times 10^{-4}$ to damp noise at the grid scale. A smaller value of $D_{4}$ leads to a slightly larger Hall reconnection rate, but does not alter our key conclusions.

\subsection{Results}

We now summarize the simulation results, followed by a careful justification of the conclusions. At early times, Sweet-Parker reconnection prevails. A secondary island first appears at $t \simeq 700$. Reconnection proceeds with the secondary island until $t \simeq 1780$, when Hall reconnection onsets. Thus, reconnection proceeds in three distinct phases including an extended phase with secondary islands but without the Hall effect triggered.

We compare the reconnection rate $E$ in the three phases to each other and to theoretical predictions in Fig. 2.1(a), showing $E$ vs. time $t$ as the solid (blue) 


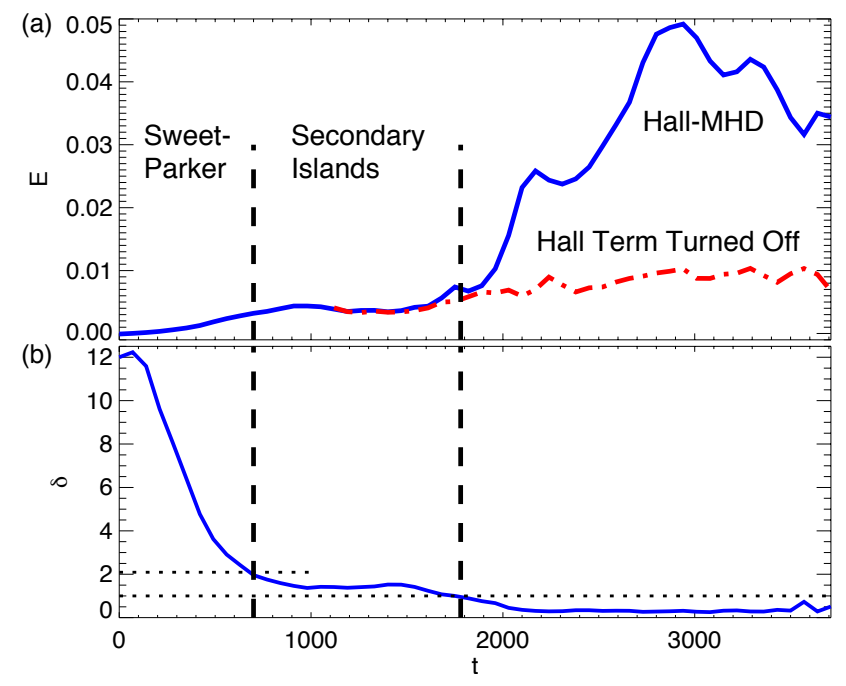

Figure 2.1: (a) Reconnection rate $E$ as a function of time $t$. The solid (blue) line is a Hall-MHD run. Dashed lines at $t \sim 700$ and 1780 indicate the onset of secondary islands and Hall reconnection, respectively. The dot-dashed (red) line shows $E$ for a simulation restarted at $t=1120$ with no Hall effect and $m_{e}=0$. (b) Thickness $\delta$ of the dissipation region vs. $t$. Horizontal dotted lines mark predicted $\delta$ for the onset of secondary islands $(\delta \sim 2)$ and Hall reconnection $(\delta \sim 1)$.

line. We measure $E$ as the time rate of change in the difference in magnetic flux function $\psi$ between the main X-line and O-line. Dashed lines at $t=700$ and 1780 denote where secondary islands and the Hall effect arise, respectively. Ignoring secondary islands, Sweet-Parker theory predicts $E \sim E_{S P} \sim 0.006$, where $E_{S P} \sim$ $\left(\eta / L_{S P}\right)^{1 / 2}$, and $L_{S P} \sim 200$. This assumes the magnetic field is its asymptotic value of 1 . The measured value is $E \sim 0.004$, slightly lower than predicted as expected because $B_{u p}<1$ (the reconnection is embedded). When $N$ X-lines are present, $E$ scales as $E_{S I} \sim E_{S P} \sqrt{N}$ [Daughton et al. (2009b); Cassak et al. (2009)]. The measured rate of 0.005 is consistent with this for a single secondary island $(N=2)$. After the Hall effect onsets, $E$ increases by about an order of magnitude. Therefore, the reconnection rate with secondary islands is faster than Sweet-Parker, 


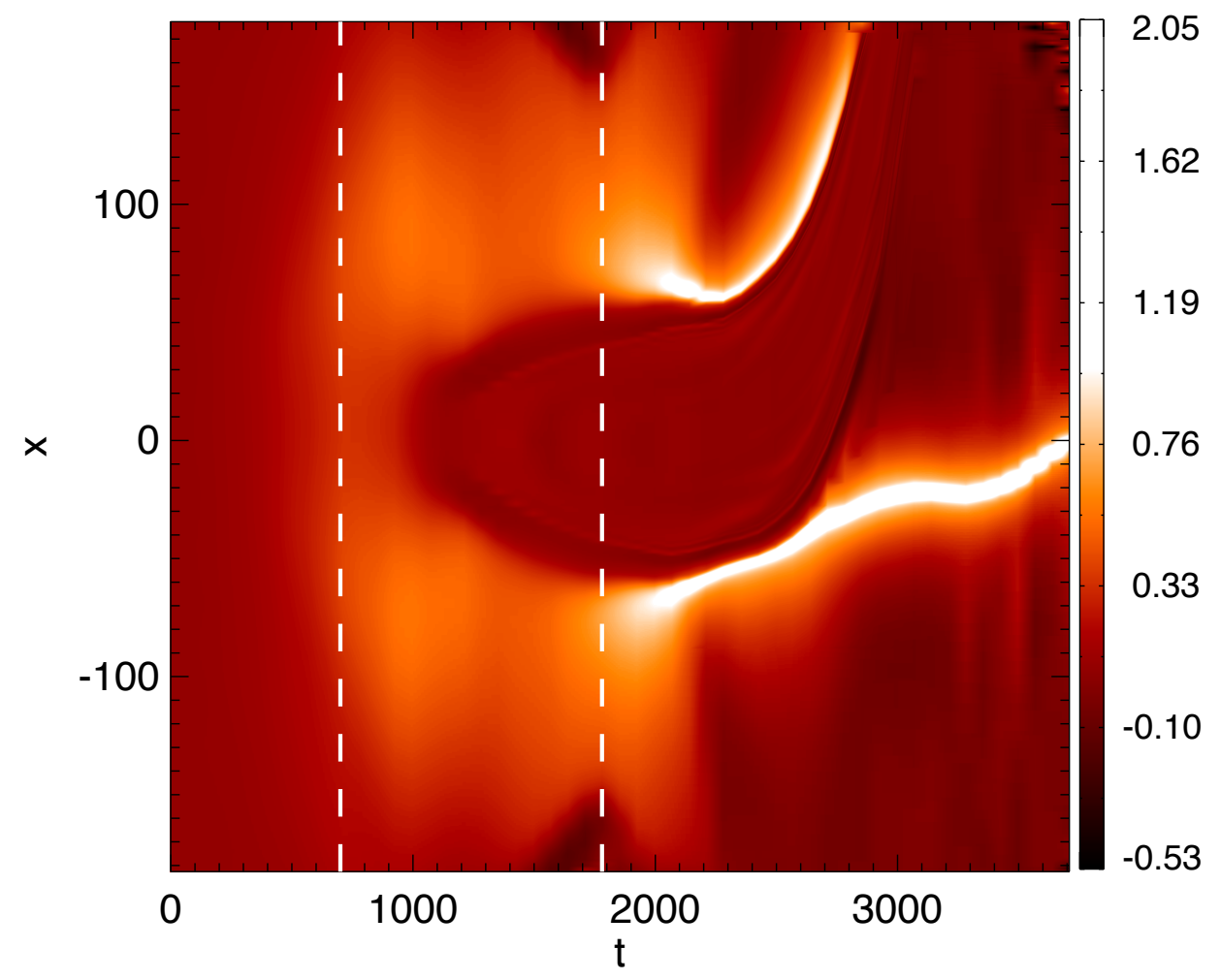

Figure 2.2: (Color) Time history plot of the out-of-plane current density $J_{z}$ in the outflow direction. Dashed lines mark when a secondary island appears and when the Hall term onsets. 
but significantly slower than Hall reconnection.

The transitions occur when predicted, as shown in Fig. 2.1(b). We plot $\delta$, measured as the half-width at half-max of $J_{z}$ in the inflow direction through the X-line, vs. $t$. The dotted lines at $\delta \sim 2$ and 1 show the predicted value when islands and the Hall effect should appear, respectively. These conditions are met at $t \simeq 700$ and 1780, in good agreement with the observed transitions.

The appearance of new physics can be seen in direct observations of the outof-plane current density $J_{z}$. A two-dimensional time history plot of $J_{z}$ in the outflow direction is plotted in Fig. 2.2. Only the half domain centered on the seeded X-line is shown. The raw data is sampled at a rate of one frame per 70 time units, so linear interpolation is used to smooth data between time slices. The effect is cosmetic, not substantive. The color bar is stretched to enhance visibility of weaker currents.

Early in time, $J_{z}$ is structureless and extends the half-length of the domain, as expected during Sweet-Parker reconnection. A secondary island near $x=0$ appears as a dark spot with associated strengthening of the fragmented current sheets. This occurs at $t \sim 700$, marked by the vertical dashed line. This agrees with Biskamp's criterion shown in Fig. 2.1(b). As time evolves, the island grows and $\delta$ shrinks. When $\delta \sim d_{i}$, Hall reconnection onsets and the current sheet becomes much shorter and intense, appearing as a sharp peak in $J_{z}$ in Fig. 2.2. This begins at $t \sim 1780$, as also marked in Fig. 2.1(b).

There are two locations where Hall reconnection onsets. An X-line near $x \simeq$ -70 onsets slightly earlier than an $\mathrm{X}$-line at $x \simeq 70$. As Fig. 2.2 vividly shows, the latter X-line is ejected from the dissipation region, along with the secondary island, 

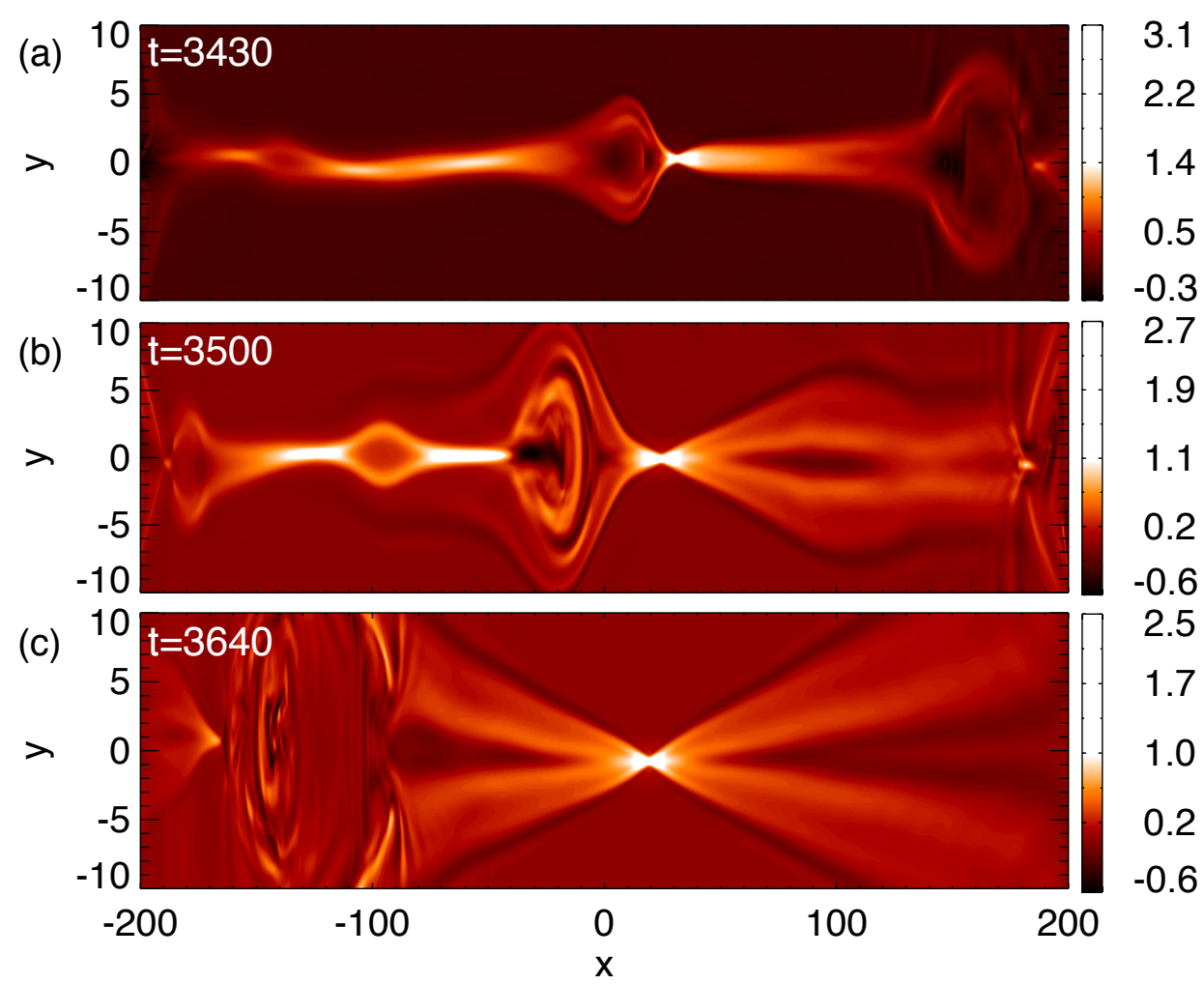

Figure 2.3: (Color) Time evolution of $J_{z}$ from the other current sheet in our double tearing mode setup, showing the ejection of secondary islands when Hall reconnection onsets. 
which is ejected at the Alfvén speed. The ejection of the secondary island implies that the two phases of reconnection will not (locally) coexist, so most of the energy is released at Hall reconnection sites.

This current sheet has only a single secondary island and one may ask whether this result remains valid in more realistic settings with multiple islands. To address this, we show results from the other current sheet in our double tearing mode setup, which self-consistently develops multiple islands. Figure 2.3 shows $J_{z}$ at three times near the onset of Hall reconnection. Panel (a) is just as Hall reconnection onsets at $x \simeq 20$, showing three pre-existing secondary islands. The Hall reconnection $\mathrm{X}$-line grows steadily, as shown in panel (b). Panel (c) shows that the single X-line at $x \simeq 20$ is the only one to persist as all of the secondary islands are ejected. This suggests that the ejection of nearby secondary islands by Hall reconnection sites is a robust result, and may reasonably represent local behavior in a macroscopic current sheet.

A careful determination of when the Hall effect begins to become important is obtained using a time history plot of the out-of-plane Hall electric field $E_{H z}=$ $J_{y} B_{x} / n$ in the inflow direction through the main X-line, plotted in Fig. 2.4(a). (Note, this cut is in the inflow direction, while Fig. 2.2 is in the outflow direction.) The color bar is again stretched. The plot clearly shows that $E_{H z}$ does not contribute during the secondary island phase. A cut of $E_{H z}$ in time, taken at the solid (gray) line in Fig. 2.4(a), is plotted in Fig. 2.4(b). The onset time, defined as when $E_{H z}$ reaches $1 \%$ of its maximum value, is at $t \sim 1780$, the time that $E$ begins to increase as seen in Fig. 2.1(a). 


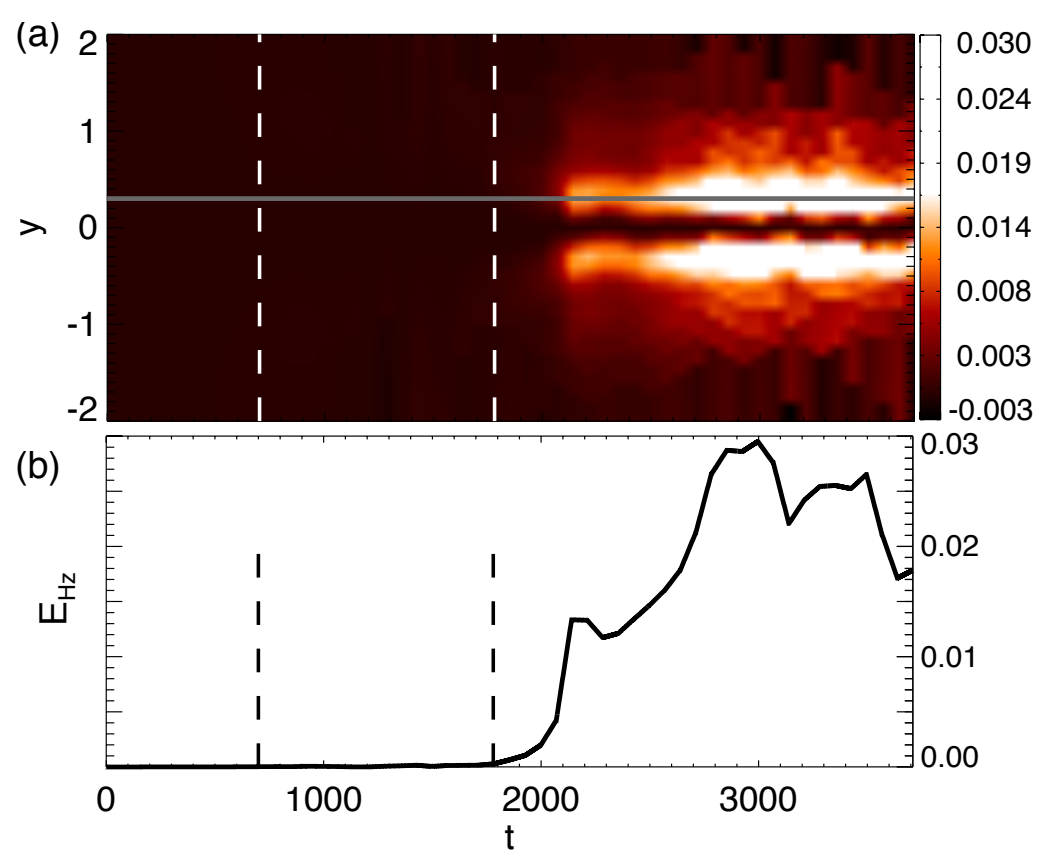

Figure 2.4: (Color) (a) Time history plot of the out-of-plane Hall electric field $E_{H z}$ in the inflow direction. The Hall term does not contribute during the secondary island phase. (b) Plot of $E_{H z}$ vs. $t$ at the $y$ location marked in panel (a).

To emphasize differences between Sweet-Parker with secondary islands and Hall reconnection, we restart the simulation at $t=1120$ with the Hall effect and electron inertia disabled. The reconnection rate is plotted as the dot-dashed (red) line in Fig. 2.1(a). The value reaches $E \sim 0.009$ as the asymptotic upstream field reaches the dissipation region, in excellent agreement with the predicted value $E_{S I} \sim$ $E_{S P} \sqrt{N} \sim 0.009$ with $N=2$ for a single island. This rate is consistent with the largest scaling studies done to date [Bhattacharjee et al. (2009)]. Note, E remains nearly an order of magnitude slower than Hall reconnection. Although the present evidence is based on simulations only up to $S \sim 10^{5}$, it is clear that secondary island reconnection does not produce the fastest reconnection rates. 


\subsection{Discussion}

In summary, reconnection in marginally collisional plasmas can evolve in three distinct phases. In particular, Sweet-Parker reconnection with secondary islands can occur without triggering collisionless effects. The reconnection rate, though faster than classical Sweet-Parker, is an order of magnitude slower than Hall reconnection. The faster rate of Hall reconnection implies that secondary islands are ejected from the dissipation region at the Alfvén speed. Due to computational constraints, the present simulations contain only a single secondary island at a time, but it is reasonable to expect that Hall reconnection sites locally eject previously existing islands in macroscopic current sheets. Thus, a majority of the magnetic energy is released at Hall reconnection sites. The transition from secondary island to Hall reconnection might be responsible for the ejection of "monster islands" [Uzdensky et al. (2010); Fermo et al. (2010)]. Monster islands are secondary islands that form near the center of the current sheet. As the monster islands are ejected they consume nearby smaller islands and plasma. Their size can reach ten percent the size of the length scale.

The present results may be relevant for observations of two-phase reconnection events in the corona. In observations of flux emergence [Longcope et al. (2005)], a slow phase of reconnection preceded an abrupt transition to a fast phase $\sim 30$ times faster (compare the slopes in their Fig. 18). In observations of the contraction of magnetic loops in an impulsive flare [Liu \& Wang (2010)], the contraction velocity abruptly increased by a factor of $\sim 16$. It is enticing to attribute these observa- 
tions to a transition from resistive secondary island reconnection at a normalized reconnection rate of $E \sim 0.01$ (consistent with implications of Refs. [Daughton et al. (2009b); Bhattacharjee et al. (2009)]) to Hall reconnection $\sim 10$ times faster, which occurs abruptly when gyroscales are reached. The existing level of accuracy of both theory and observations make such an identification premature, but it remains an exciting possibility. 


\section{Chapter 3}

\section{Guide Field Dependence of 3D X-line Spreading During Collisionless}

\section{Magnetic Reconnection}

The previous chapter detailed the transition from Sweet-Parker to SweetParker enhanced by secondary islands to Hall magnetic reconnection. The study was performed in a two-dimensional box, but in nature it is difficult to imagine a scenario in which reconnection is a perfectly two-dimensional process. Magnetic reconnection observed in the solar corona, magnetotail, and laboratory experiments often exhibits behavior of beginning in a localized region and then spreading in the direction perpendicular to the plane of reconnection. We refer to this behavior as X-line spreading. A sketch of reconnection spreading is shown in Fig. 3.1. As discussed later in this chapter, the mechanism of X-line spreading is very sensitive to the strength of the out-of-plane or guide field. Depending on where reconnection is taking place, e.g., the corona versus the magnetotail, the relative strength of the guide field varies greatly.

This chapter addresses reconnection spreading with and without a guide field, and the effect of the guide field on spreading. In Section 3.1, we discuss previous theoretical and observational work on X-line spreading. In Section 3.2, we introduce a theory of X-line spreading which predicts the behavior of reconnection spreading depending on the strength of the guide field. In Section 3.3, the three-dimensional 


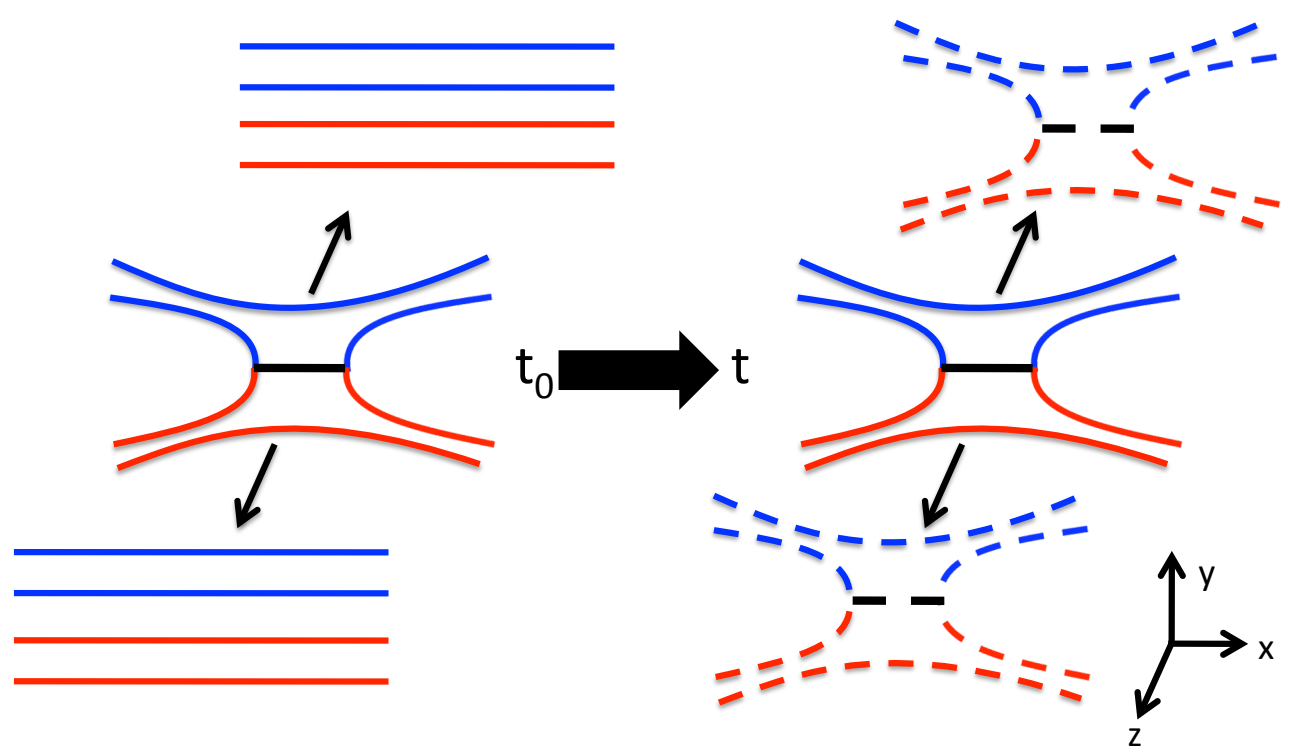

Figure 3.1: A sketch of magnetic reconnection spreading. At initial time $t_{0}$, magnetic reconnection is only occurring at the center of the domain. Some time $t$ later, magnetic reconnection spreads in the out-of-plane direction and allows excites new reconnection sites.

simulation setup that will be used to test our predictions is discussed. In Section 3.4, the results of the simulation that confirm our predictions are presented. The entirety of the work presented in this chapter was published in the Journal of Geophysical Research in October 2012 [Shepherd \& Cassak (2012)].

\subsection{Introduction}

Early models [Sweet (1958); Parker (1957); Petschek (1964)] and the predominance of numerical work on magnetic reconnection (e.g., [Birn et al. (2001)]) have treated reconnection as two-dimensional. However, naturally occurring magnetic reconnection often begins in a localized region and spreads in the direction perpendicular to the plane of reconnection. For example, satellite observations of sub- 


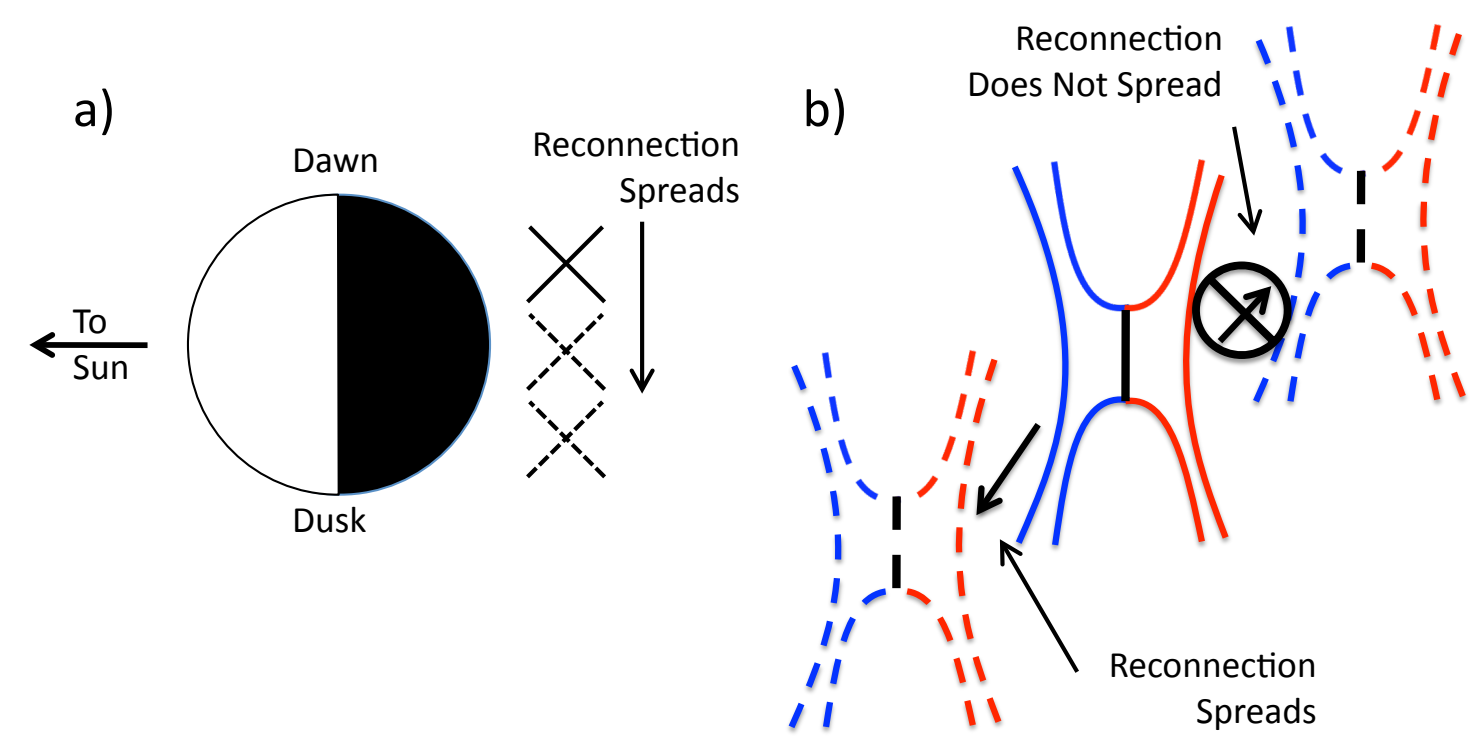

Figure 3.2: (a) The dawn-dusk asymmetry in the magnetotail. Magnetic reconnection spreads exclusively in the duskward direction. (b) The dawn-dusk asymmetry in the solar corona. Solar arcade formation favors one direction over the other.

storms in the magnetotail identified a dawn-dusk asymmetry caused by localized reconnection spreading in the westward direction [McPherron et al. (1973); Nagai (1982); Nagai et al. (2013)]. The dawn-dusk asymmetry is shown in Fig. 3.2(a) in the magnetotail. Reconnection begins in a localized region (solid-X) and spreads duskward (dashed-X). A similar asymmetry was observed in the formation of arcades in the solar corona [Isobe et al. (2002)]. This can be visualized in Fig. 3.2(b), where reconnection begins on the arcade in a localized region and favors spreading uni-directionally. Capturing effects such as these requires a fully three-dimensional treatment.

A number of numerical studies have addressed X-line spreading in the direction of the current during quasi-two-dimensional reconnection. Using a magnetic perturbation localized in the out-of-plane direction in Hall-magnetohydrodynamics 
(Hall-MHD), the localized reconnection signal was found to propagate as a wave structure carried by the electron current [Huba \& Rudakov $(2002,2003)]$. By seeding reconnection with large random magnetic perturbations in Hall-MHD simulations, it was observed that reconnection develops into spatially isolated structures which lengthen in the direction of the electron current and these small structures merge into larger scale structures [Shay et al. (2003)]. This study suggested that spreading occurs in the direction of whichever species carries the current, which need not be exclusively electrons. Spreading by the ions when they carry the current was observed in hybrid simulations with localized resistivity [Karimabadi et al. (2004)]. These works demonstrate reconnection X-line spreads in the out-of-plane direction by the current carriers in the direction of the current carriers [Lapenta et al. (2006)]. Nakamura et al. (2012) presented the first systematic study to vary the fraction of current carried by each of the species; the results confirmed X-line spreading occurs due to the current carriers. The results are not dependent on the Harris sheet geometry; [Lukin \& Linton (2011)] observed X-line spreading in simulations of island coalescence. Note, each of these studies primarily favored magnetotail applications, so they either treated anti-parallel reconnection or reconnection with a weak outof-plane (guide) magnetic field compared to the background field. X-line spreading in a system without a guide field was recently observed in laboratory experiments at the Magnetic Reconnection eXperiment (MRX), and a physical mechanism for spreading by current carriers was proposed [Dorfman (2012)].

Interestingly, experimental and satellite observations of systems with a strong guide field reveal strikingly different behavior of X-line spreading. For example, 


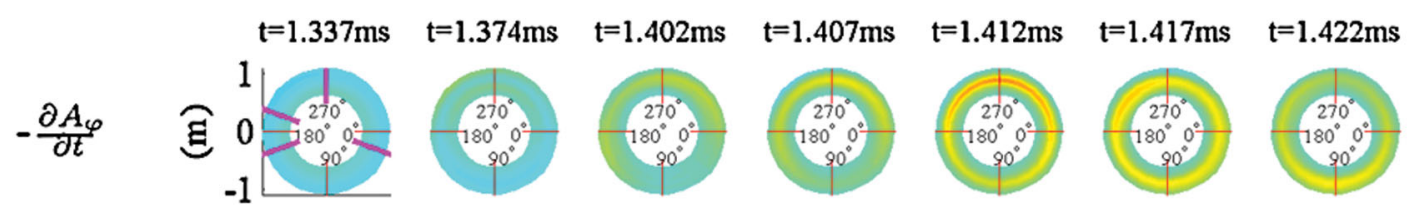

Figure 3.3: Measurement of the reconnection rate as a function of time. The increase in the reconnection rate is shown as the transition from ligt blue to red. Printed with permission from Katz et al. (2010). (c) 2010 by The American Physical Society.

experiments performed at the Versatile Toroidal Facility (VTF) [Katz et al. (2010);

Egedal et al. (2011)] exhibit reconnection beginning in a localized region and spreading bi-directionally in the out-of-plane (toroidal) direction at a speed consistent with the Alfvén speed based on the guide field. Figure 3.3 is a plot of the reconnection rate as a function of time from the Katz et al. (2010) study. Magnetic reconnection begins around $\phi \sim 270^{\circ}$ at $t=1.374 \mathrm{~ms}$ and then spreads bi-directionally at the Alfvén speed attributed to the guide field. Another example is bi-directional spreading (or elongating) of ribbons observed during two-ribbon solar flares [Qiu (2009)] shown in Fig. 3.4(a), including the Bastille Day flare [Qiu et al. (2010)] in Fig. 3.4(b). This presumably is related to spreading of the looptop reconnection site where a sizable guide field is expected to be present because of the $3 \mathrm{D}$ nature of the reconnection located in the corona. This spreading was also inferred to take place at the local Alfvén speed. Prominence eruptions in the corona have also been observed to spread bi-directionally; this behavior was attributed to magnetic reconnection propagating along the magnetic polarity inversion line (PIL), where the magnetic field flips direction at the coronal loop top [Tripathi et al. (2006)]. In magnetospheric contexts, observations of extended X-lines several Earth radii long at 


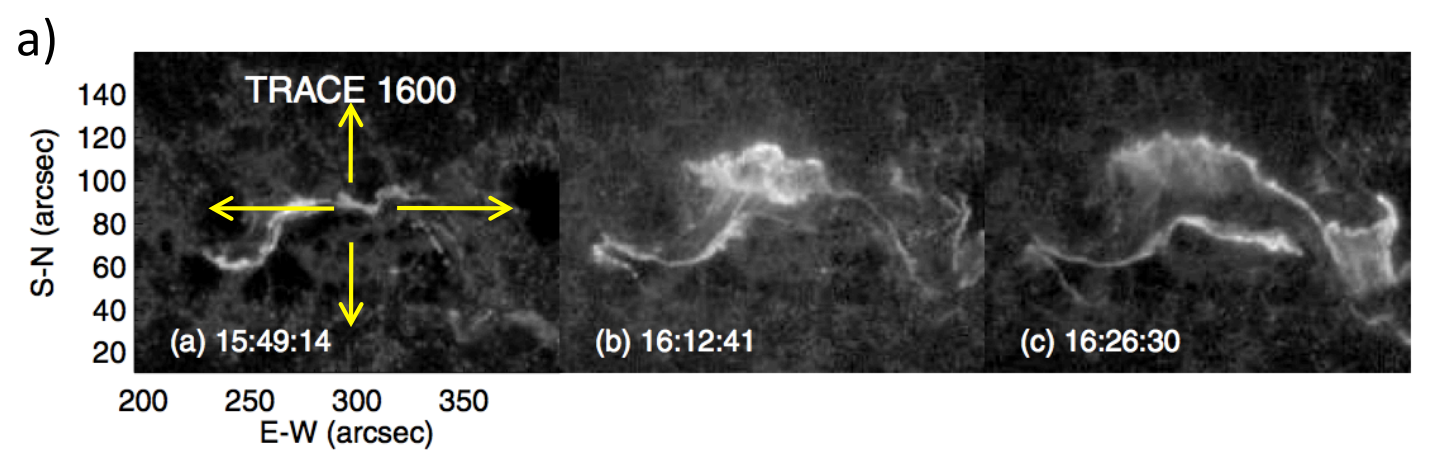

\section{b)}
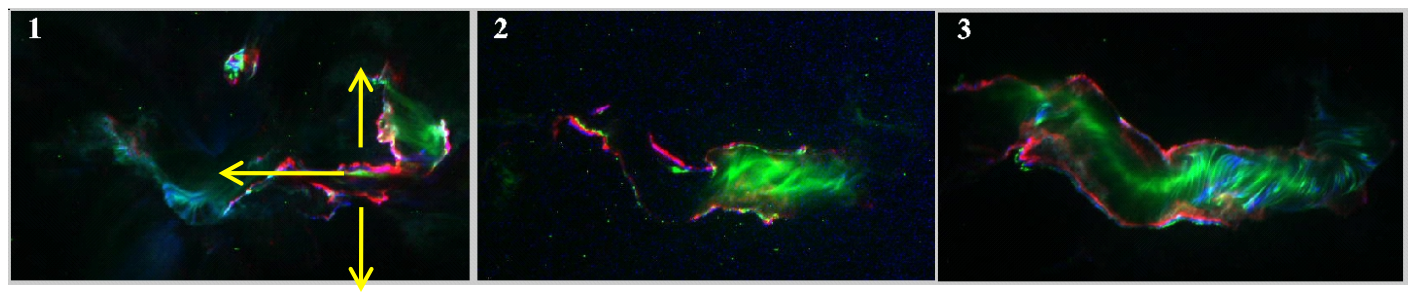

Figure 3.4: (a) Time evolution of a two-ribbon flare from November 7, 2004. The initial flare region expands into the $\mathrm{E}-\mathrm{W}$ and $\mathrm{S}-\mathrm{N}$ directions (indicated by the yellow lines). Adapted from Qiu (2009). (c) 2009 by The American Astronomical Society. (b) The well known Bastille day flare that occurred on July 14, 2000. There is rapid expansion again favoring expansions towards the left. Image Credit: NASA/TRACE.

the magnetopause [Phan et al. (2000); Fuselier et al. (2002)] and hundreds of Earth radii in the solar wind [Phan et al. (2006)] suggest X-line spreading occurs in these areas as well, although direct evidence of spreading is prohibitively difficult with single- or even multi-point satellite observations. X-line spreading was also seen in three-dimensional two-fluid simulations with a guide field [Schreier et al. (2010)].

The existing observational data provide a clear indication that the mechanism controlling X-line spreading strongly depends on the strength of the guide field. In the weak guide field limit, the signal is transmitted by the current carriers; in the strong guide field limit, the reconnection signal is transmitted by the magnetic field as an Alfvén wave. In general, we hypothesize the X-line spreads in both directions 
at the speed of whichever mechanism is faster for that direction. We emphasize, we are considering thin current sheets with large amounts of free magnetic energy present. The important topics of how the sheets become thin and how the magnetic energy is stored is outside the scope of this study.

\subsection{Theory}

Here, we develop a prediction of the speed at which the X-line spreads in each out-of-plane direction as a function of guide field and derive the critical guide field at which the mechanism causing the spreading changes from current carriers to Alfvén waves. To do so, we make the following simplifying assumptions. We treat a quasi-two-dimensional system, meaning that the equilibrium parameters do not depend strongly on the direction normal to the reconnection plane for all time. We assume the current layer is flat, so that the current sheet is either not curved or that the curvature does not strongly contribute to the dynamics. We assume the plasma parameters are symmetric on either side of the current layer; asymmetries [Cassak \& Shay (2007)] are not considered here. Finally, we assume that a single mode dominates the dynamics; in previous simulations, it was shown that when multiple modes of reconnection occur, they can impede the spreading of X-lines [Schreier et al. (2010)]. This assumption is valid at early times and in systems in which only a single mode is present.

First, we estimate the spreading speed in each direction for each spreading mechanism. We begin with the speed due to the current carriers. From Ampère's 
law, the current is $\mathbf{J}=c \nabla \times \mathbf{B} / 4 \pi$, where $\mathbf{B}$ is the magnetic field. For simplicity, we first assume the electrons carry the out-of-plane current, so that the electron velocity is $\mathbf{v}_{e}=-\mathbf{J} / n e$, where $n$ is the electron density and $e$ is the proton charge. Using a scaling argument, the electron speed $v_{e g}$ in the out-of-plane direction is

$$
v_{e g} \sim \frac{c B_{r e c}}{4 \pi n e \delta}
$$

where $B_{\text {rec }}$ is the strength of the reconnecting magnetic field upstream of the electron layer, $\delta$ is the thickness of the current layer, and $g$ refers to the direction of the guide field. As has been previously established [Huba \& Rudakov (2002); Shay et al. (2003); Karimabadi et al. (2004); Lapenta et al. (2006); Lukin \& Linton (2011); Nakamura et al. (2012)], this is the X-line spreading speed in the absence of a guide field. In the strong guide field limit, the observations suggest the spreading speed is the Alfvén speed $c_{A g}$ based on the guide field, given by

$$
c_{A g}=\frac{B_{g}}{\sqrt{4 \pi m_{i} n}}
$$

where $B_{g}$ is the strength of the guide field and $m_{i}$ is the proton mass.

Our hypothesis is that the X-line spreading speed in the direction of the electron out-of-plane flow, which we call $v_{X e}$, is the larger of $v_{e g}$ and $c_{A g}$ :

$$
v_{X e}=\max \left\{v_{e g}, c_{A g}\right\}
$$

From this, one can find the critical guide field $B_{c r i t, e}$ at which the spreading mech- 
anism changes, where the $e$ subscript denotes the critical field for motion in the direction of the out-of-plane electron flow. Setting Eq. (3.1) equal to Eq. (3.2) and solving for $B_{g}$ gives

$$
B_{\text {crit }, e} \sim B_{r e c} \frac{d_{i}}{\delta}
$$

where $d_{i}=c / \omega_{p i}$ is the ion inertial length and $\omega_{p i}=\left(4 \pi n e^{2} / m_{i}\right)^{1 / 2}$ is the ion plasma frequency. Since $\delta$ is typically less than $d_{i}$ as the current is set by electron scales, we expect $B_{\text {crit,e }}>B_{\text {rec }}$. $B_{\text {crit }, e}$ is on the order of and slightly larger than the reconnecting magnetic field strength upstream of the ion dissipation region.

We perform a similar analysis for the spreading speed in the direction of the ions $v_{X i}$. Since electrons carry the current, the ion speed $v_{i g}$ in the out-of-plane direction is

$$
v_{i g}=0
$$

Therefore, the X-line spreading speed in the direction of the ion out-of-plane flow $v_{X i}=\max \left\{v_{i g}, c_{A g}\right\}$ is given by the Alfvén speed based on the guide field,

$$
v_{X i}=c_{A g}
$$

Since $v_{i g}=0$, the critical guide field $B_{c r i t, i}$ for spreading in the direction of the ion out-of-plane flow is

$$
B_{\text {crit }, i}=0
$$

These results can be generalized to systems with both electrons and ions carrying some of the current. Following Nakamura et al. (2012), we define the fraction of 
the total current $J_{z}$ carried by the ions as $\alpha$, which is assumed known or measurable. Letting $J_{i z}=\alpha J_{z}$, one has $J_{e z}=(1-\alpha) J_{z}$ so that $J_{z}=J_{i z}+J_{e z}$. By performing a similar analysis as before, one finds the out-of-plane electron and ion flow speeds due to current carrying are

$$
\begin{aligned}
v_{e g} & \sim(1-\alpha) \frac{c B_{r e c}}{4 \pi n e \delta}, \\
v_{i g} & \sim \alpha \frac{c B_{r e c}}{4 \pi n e \delta}
\end{aligned}
$$

which generalizes Eqs. (3.1) and (3.5). The X-line spreading speeds in the direction of the electron and ion out-of-plane flow are

$$
\begin{aligned}
& v_{X e}=\max \left\{v_{e g}, c_{A g}\right\}, \\
& v_{X i}=\max \left\{v_{i g}, c_{A g}\right\},
\end{aligned}
$$

respectively. Finally, the critical guide fields at which the mechanism for X-line spreading changes from the current carriers to Alfvén waves in the direction of electron and ion flows are given by

$$
\begin{aligned}
B_{\text {crit }, e} & \sim(1-\alpha) B_{r e c} \frac{d_{i}}{\delta} \\
B_{c r i t, i} & \sim \alpha B_{r e c} \frac{d_{i}}{\delta}
\end{aligned}
$$

respectively, which generalizes Eqs. (3.4) and (3.7).

The predictions derived here are summarized pictorially in Fig. 3.5, where the 


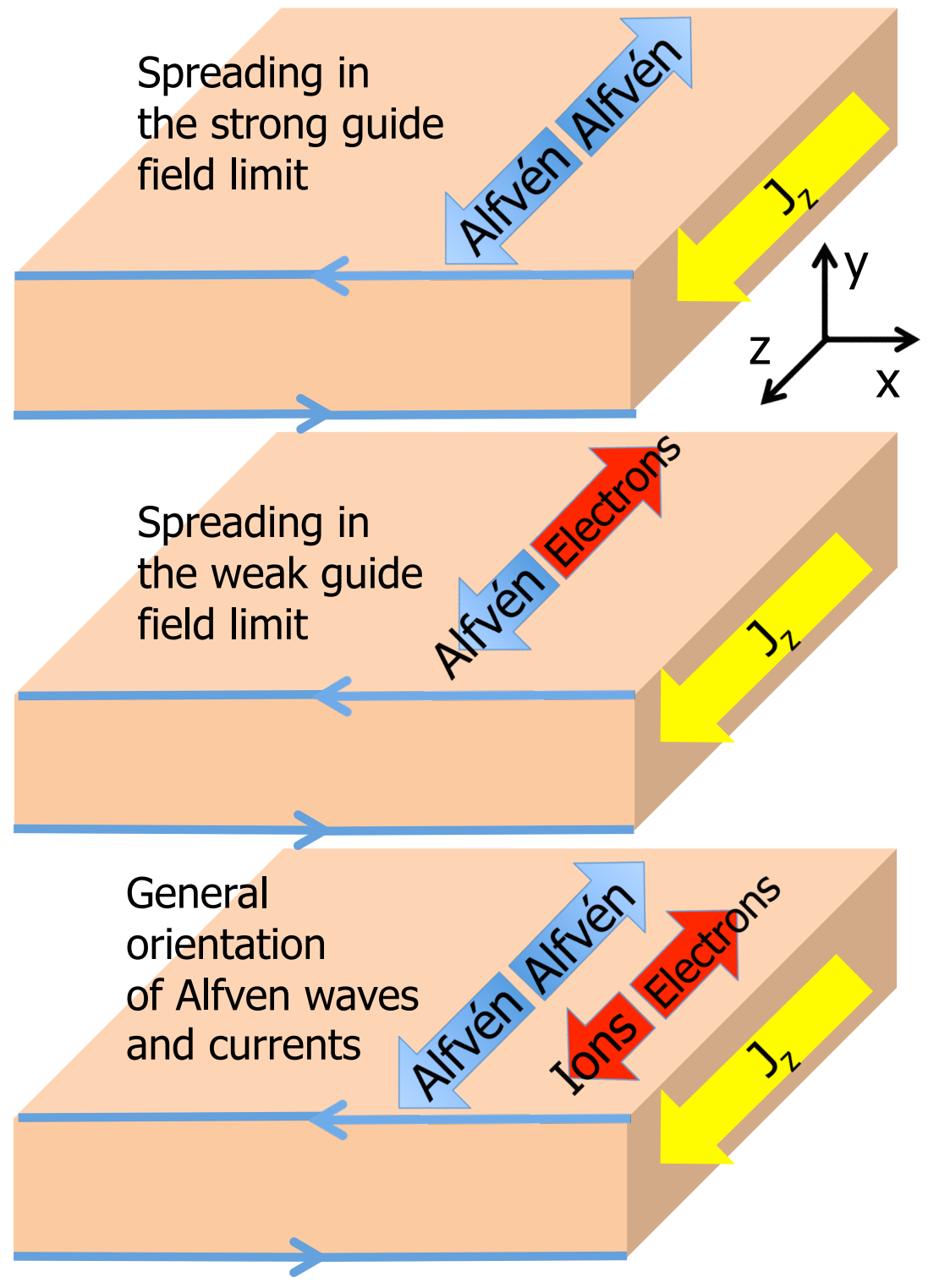

Figure 3.5: Schematic diagram showing the mechanisms that cause X-line spreading. The thin blue arrows are the reconnecting magnetic field components, the yellow arrow is the total current. The red arrows denote the speed of the current carriers; the thick blue arrows denote the speed of Alfvén waves along the guide field. The top, middle, and bottom diagrams show the spreading mechanisms for strong, weak, and intermediate guide field strengths. In each case, X-line spreading occurs at the faster speed in each direction. 
current is depicted by the yellow arrows and the reconnecting magnetic fields are the thin blue lines. The thick arrows denote the speeds of the current carriers (in red) and the Alfvén speed (in blue) in each out-of-plane direction. The top, middle, and bottom plots show the results for strong, weak, and intermediate guide field strengths, respectively. In each case, the X-line spreading speed is the longer of the arrows on either side. Note, there is nothing preventing the spreading mechanisms from being different in the two directions, i.e., Alfvén waves in one direction and current carriers in the other, if that is what Eq. (3.10) dictates for the system parameters.

\subsection{Simulation Setup}

To test the predictions of X-line spreading, three-dimensional numerical simulations are performed using the two-fluid code F3D [Shay et al. (2004)]. The code updates the continuity, momentum, and induction equations with the generalized Ohm's law including electron inertia. Magnetic fields and densities are normalized to arbitrary values $B_{0}$ and $n_{0}$. Velocities are normalized to the Alfvén speed $c_{A 0}=B_{0} /\left(4 \pi m_{i} n_{0}\right)^{1 / 2}$. Lengths are normalized to the ion inertial length $d_{i 0}=c / \omega_{p i 0}=\left(m_{i} c^{2} / 4 \pi n_{0} e^{2}\right)^{1 / 2}$. Times are normalized to the ion cyclotron time $\Omega_{c i 0}^{-1}=\left(e B_{0} / m_{i} c\right)^{-1}$, electric fields to $E_{0}=c_{A 0} B_{0} / c$, and temperatures to $T_{0}=$ $m_{i} c_{A 0}^{2}$

Simulations are performed in a three-dimensional domain of size $L_{x} \times L_{y} \times L_{z}=$ $51.2 \times 25.6 \times 256.0 d_{i 0}$, where $x$ is the direction of the oppositely directed field, $y$ 
corresponds to the inflow direction if the simulations were two-dimensional, and $z$ is the direction of the initial current. The plasma is assumed to be isothermal and there is no resistivity $(\eta=0)$. Boundaries in all three directions are periodic, but the system is long enough in the $z$ direction that the periodic boundaries do not affect the dynamics on the time scales of import to the present study.

For simplicity, the simulations have the electrons carrying all of the initial current (i.e., $\alpha=0$ ). The electron inertia is $m_{e}=m_{i} / 25$. In previous simulations with this electron mass (and confirmed in the simulations here), it has been observed that the current layer thickness $\delta$ thins down to the electron inertial scale $d_{e}=0.2 d_{i}$ and the reconnecting magnetic field at the electron layer is $B_{\text {rec }} \simeq 0.4 B_{0}$ [Jemella et al. (2003)]. Substituting this into Eq. (3.4), we predict a critical guide field of

$$
B_{\text {crit }} \simeq 2 B_{0}
$$

Therefore, we can test the theory by running a series of simulations in which the initial guide fields are $B_{g}=0,0.5,1,1.5,2,2.5$ and 3 . Note, the scaling $\delta \sim d_{e}$ and $B_{\text {rec }} \sim 0.4 B_{0}$ may or may not be representative of naturally occurring reconnection; care should be taken to investigate this for particular applications.

The initial configuration is a double tearing mode with two Harris sheets, $B_{x 0}(y)=\tanh \left[\left(y+L_{y} / 4\right) / w_{0}\right]-\tanh \left[\left(y-L_{y} / 4\right) / w_{0}\right]-1$, with uniform initial temperature $T=1$ and a non-uniform plasma density to balance total pressure. Here, $w_{0}=0.4 d_{i 0}$ is the initial current layer thickness. We choose this thickness to be comparable to the smallest value of the ion Larmor radius $\rho_{s}=c_{s} / \Omega_{c i}=\sqrt{T} / B_{g} \simeq 0.33$, 
where $c_{s}$ is the sound speed and the latter expression is written in normalized units. This scale is the Hall scale in the presence of a strong guide field [Zakharov et al. (1993); Rogers et al. (2001)]. It is worth noting that the Hall scale increases smoothly from $\rho_{s}$ to $d_{i}$ as the guide field is decreased to zero, which follows from a linear analysis of Hall-MHD waves [Rogers et al. (2001)]. Consequently, the smaller guide field simulations start with a current sheet that is thin relative to the Hall scale, and should onset rapidly. As the guide field is increased, the time to onset should increase and it is expected that a hyper-resistive phase of reconnection will occur before onset. This behavior will not adversely impact our study, as we will separate out the times for which Hall reconnection is dominant.

We employ a grid scale of $\Delta x \times \Delta y \times \Delta z=0.05 \times 0.05 \times 1.0 d_{i 0}$. Using a stretched grid in the out-of-plane direction has been done before [Shay et al. (2003)], and is acceptable since the in-plane kinetic-scale dynamics is on smaller scales than the out-of-plane dynamics. To ensure the stretched grid scale in the out-of-plane direction does not play a role in the numerics, some simulations are confirmed by comparison with simulations with $\Delta z=0.5 d_{i 0}$. All equations employ a fourth-order diffusion with coefficient $D_{4 x}=D_{4 y}=2.5 \times 10^{-5}$ in the $x$ and $y$ directions. In the out-of-plane direction the fourth-order diffusion coefficient $D_{4 g}$ depends on the speeds in the out-of-plane direction. For $B_{g} \leq 2.0$ the fourthorder diffusion coefficient is $D_{4 g}=0.064$ and for $B_{g}=2.5$ and 3.0 the fourth-order diffusion coefficient is $D_{4 g}=0.081$ and 0.097 , respectively. The values of $D_{4 g}$ were tested by varying the value by a factor of two to ensure that $D_{4 g}$ does not play a significant role in the dynamics. 
The inclusion of a guide field in these simulations changes the nature of reconnection relative to previous work on X-line spreading. In three-dimensional periodic domains, it is well established that the linear tearing instability is excited where $\mathbf{k} \cdot \mathbf{B}_{0}=0$, where $\mathbf{B}_{0}=B_{x 0} \hat{\mathbf{x}}+B_{z 0} \hat{\mathbf{z}}$ is the equilibrium magnetic field and $\mathbf{k}=k_{x} \hat{\mathbf{x}}+k_{z} \hat{\mathbf{z}}$ is the wave vector of the mode. The periodic domain enforces that $k_{x}=2 \pi m / L_{x}$ and $k_{z}=2 \pi n / L_{z}$, where $m$ and $n$ are integer mode numbers which specify the number of X-lines in the $x$ and $z$ direction, respectively. In the absence of a guide field, this condition is only satisfied where $B_{x 0}=0$. With a guide field, it is satisfied wherever $q(y)=L_{x} B_{z}(y) / L_{z} B_{x}(y)=m / n$ is a rational number, where $q(y)$ is the safety factor well known in fusion applications. The $y$ locations where $\mathbf{k} \cdot \mathbf{B}_{0}=0$ is satisfied are called rational surfaces, and for the equilibrium profile here, the modes are displaced from where $B_{x 0}=0$ by a distance $y_{s}=w_{0} \tanh ^{-1}\left(n L_{x} B_{g} / m L_{z} B_{0}\right)$. Thus, modes in our simulations are excited on multiple rational surfaces.

Reconnection is seeded using a coherent magnetic perturbation localized in the out-of-plane direction of the form

$$
B_{y 1}=\sum_{k_{x}, k_{z}} \widetilde{B}_{1} \sin \left(k_{x} x+k_{z} z\right) f_{z}(z)
$$

where $\widetilde{B}_{1}=0.1$. Here, $f_{z}(z)$ is an envelope that localizes the perturbation in the outof-plane direction and is given by $f_{z}(z)=\left\{\tanh \left[\left(z+w_{0 z}\right) / 6\right]-\tanh \left[\left(z-w_{0 z}\right) / 6\right]\right\} / 2$. We use $w_{0 z}=1$; a plot of $f_{z}(z)$ is in Fig. 3.6. Random magnetic perturbations that range from $m, n=0$ to 20 with small amplitude $0.02 B_{0}$ are included with the 


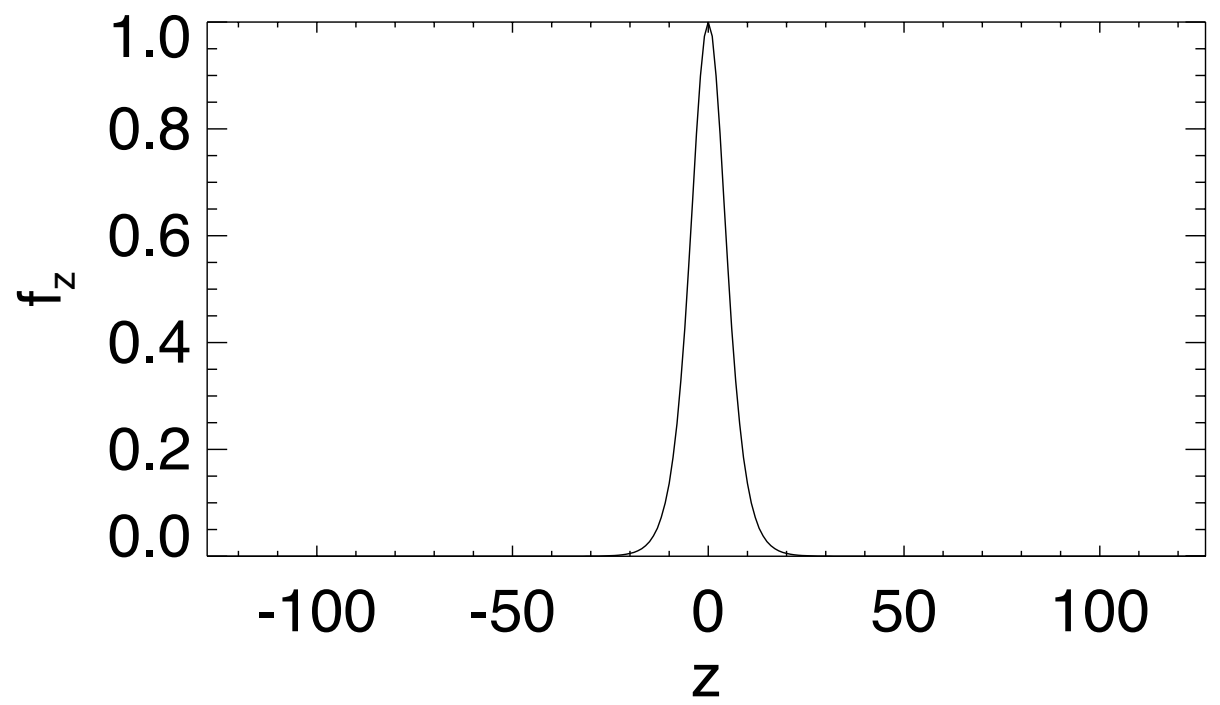

Figure 3.6: The envelope $f_{z}(z)$ used to localize the magnetic perturbation in the out-of-plane direction $z$.

initial conditions to break symmetry so that secondary islands are ejected.

In early simulations, we initially excite only the $(m, n)=(1,0)$ mode in Eq. (3.12). Even though this mode is the strongest perturbation, oblique modes with $n \neq 0$ grow from the noise and dominate the reconnection. This is consistent with recent particle-in-cell (PIC) simulations [Daughton \& Roytershteyn (2011)] and linear theory [Baalrud et al. (2012)]. Oblique modes in reconnection have been observed many times in fusion applications (see e.g., [Grasso et al. (2007)]). In light of these results, we include oblique modes in Eq. 3.12) and compare the results with the original simulations. The values of $m$ and $n$ are chosen so that the displacement $y_{s}$ is less than $w_{0}$. In this study, $m=1$ for all simulations and $n$ ranges from 0 to 3. Initially exciting oblique modes has no noticeable effect on the results on the development of reconnection. Thus, the results of this study are expected to be independent of the modes used to seed reconnection. Note, although the modes are 
oblique, they are still quasi two-dimensional until they start interacting strongly. It was shown [Schreier et al. (2010)] that interacting oblique modes can prevent X-lines from spreading, so we focus on times early enough in the evolution that oblique mode interactions have not yet occurred.

\subsection{Results}

To ensure numerical feasibility of the simulations in three dimensions, we benchmark the simulations in two dimensions. The simulations are evolved from $t=0$ until nonlinear reconnection develops. In two dimensions, symmetry dictates that the $n=0$ mode is the only excited mode. The reconnection rate $E$, measured as the time rate of change of the difference in magnetic flux between the X-line and O-line during a quasi-steady period, is approximately $0.08-0.1$ for all simulations. Also, as expected, the time until Hall reconnection begins increases as the guide field increases since $w_{0}$ is held fixed, and there is a brief hyper-resistive reconnection phase before onset for stronger guide fields.

In the three-dimensional simulations, the evolution at $z=0$ is very similar to what is observed in two dimensions: the time scale of the development of reconnection is comparable, and a hyper-resistive phase precedes Hall reconnection. The reconnection rates can be compared, as well. The reconnection rate in threedimensions is measured by taking a cut of the $z$ component of $\mathbf{v} \times \mathbf{B}$ in the $y$-direction across the X-line; far from the current sheet, it asymptotes to the reconnection electric field in a steady-state. The reconnection rates are in the 0.08-0.1 range, 

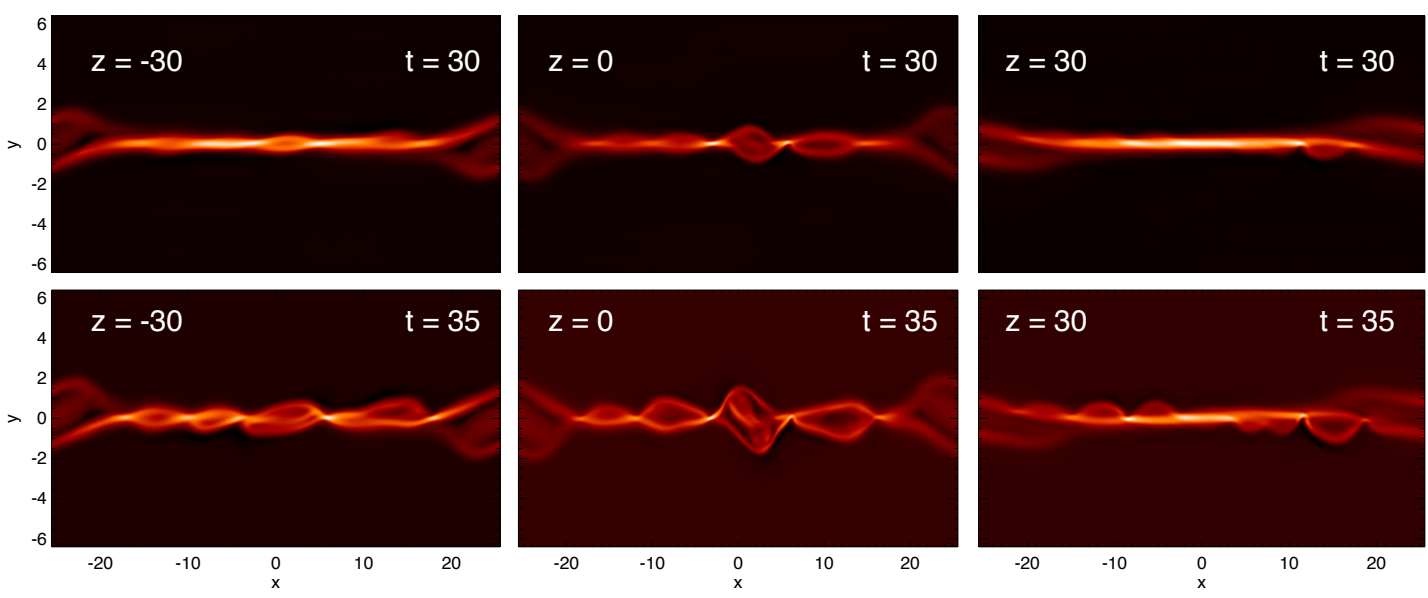

Figure 3.7: Cuts at different values of $z$ of the out-of-plane current $J_{z}$ for the $B_{g}=3$ simulation. At $t=30$ (top row), Hall reconnection is developing at $z=0$ but not at $z= \pm 30$. At $t=35$ (bottom row), Hall reconnection has developed fully at $z=0$ and is developing at $z= \pm 30$.

comparable to the two-dimensional results. One noticeable difference, as discussed earlier, is that oblique modes dominate over $n=0$ modes in three-dimensions.

Each of the three-dimensional simulations display some form of X-line spreading. This can be seen qualitatively in Fig. 3.7 for the simulation with $B_{g}=3$. The out-of-plane current $J_{z}$ at time $t=30$ (top row) and at time $t=35$ (bottom row) is displayed at three different out-of-plane positions: $z=-30,0$, and 30 from left to right. At the earlier time $t=30$, a transition to fast (Hall) reconnection at multiple sites at $z=0$ has occurred, consistent with the development of multiple oblique modes. At $z= \pm 30$, the reconnection is still hyper-resistive. At the later time $t=35$, the current sheet at all three positions in $z$ has developed multiple Hall reconnection X-lines. Thus, the Hall reconnection signal propagates bi-directionally from $z=0$ for $B_{g}=3$. It is worth noting that the multiple oblique mode reconnection seen here is consistent with previous simulations, and the reason multiple 

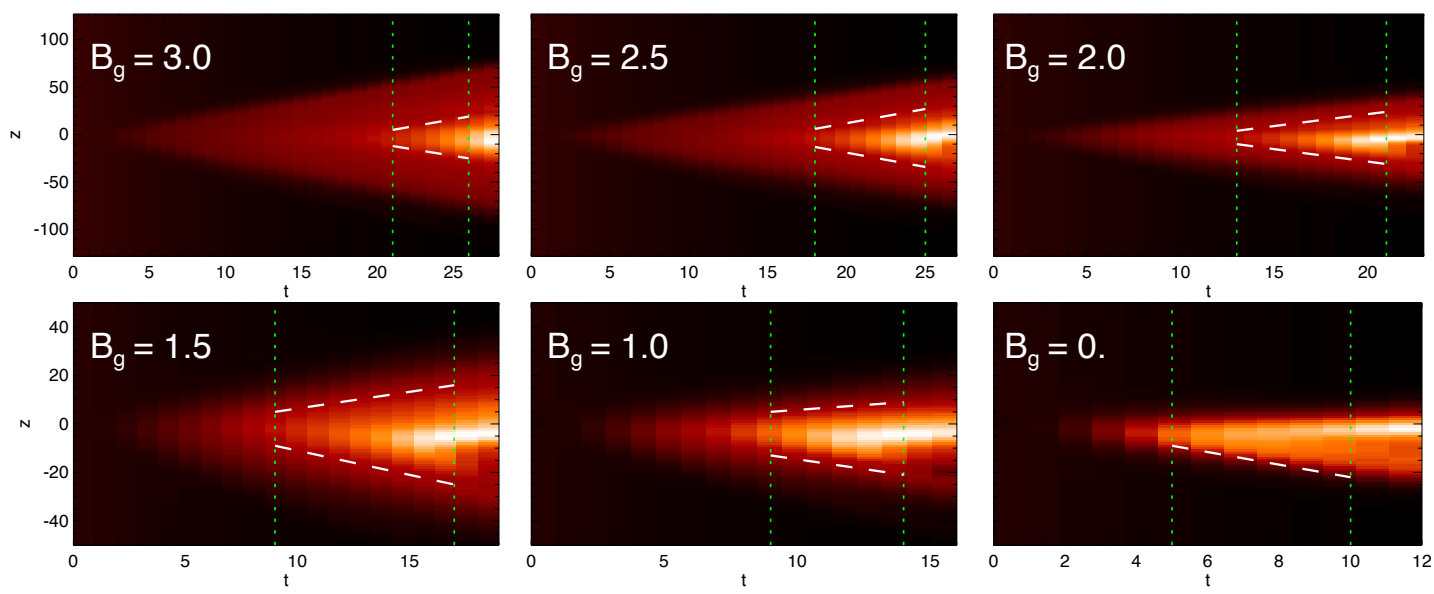

Figure 3.8: Stack plots of $J_{\max }(z)$ as a function of $t$ and $z$. The vertical dashed green lines indicate the range of time over which the spreading speed is measured, $t_{i}$ and $t_{f}$. The dashed white lines indicate the extent in $z$ of the X-line; their slope gives the speed of the spreading. Note, the images in the bottom row are on a different scale in $z$ than those in the top row.

X-lines appear despite the $m=1$ mode being the dominant mode is that there are multiple modes simultaneously excited on different rational surfaces.

To quantify the speed at which the X-line spreads, we must develop a systematic way to determine the extent of the reconnection region. As Hall reconnection develops, the out-of-plane current $J_{z}$ at the X-line becomes noticeably higher than regions where reconnection is hyper-resistive. For each slice in $z$, we measure the maximum out-of-plane current, which we call $J_{\max }(z)$. These maximum values of the current correspond to the location of the X-line for each position in $z$. The extent of the X-line can then be readily seen in a stack plot of $J_{\max }(z)$ as a function of t. Stack plots for all six initial guide fields in this study are displayed in Fig. 3.8. As mentioned earlier, the plots only cover early times when the three-dimensional Xline structure is well defined because the interaction of oblique modes make defining 
the X-line structure prohibitive.

The bright white regions in Fig. 3.8 correspond to the strongest currents and, thus, the Hall reconnection X-lines. The dimmer areas outside of the white dashed lines (the red to black colors) indicate the region undergoing hyper-resistive reconnection. As expected, the $B_{g}=0$ simulation onsets almost immediately without a hyper-resistive phase since $w_{0}<d_{i}$, while the onset time increases as the guide field increases, leading to a longer hyper-resistive phase. Both phases of reconnection spread in the $z$ direction as time evolves; we focus on the Hall reconnection X-lines in the present study.

From Fig. 3.8, the qualitative differences in the nature of the spreading as a function of the guide field can readily be seen. For the strong guide field simulations $B_{g} \geq 2$, the $\mathrm{X}$-line spreads symmetrically about $z=0$ (top row), which is consistent with our expectations for the strong guide field regime from Eq. (3.11). However, for simulations with a guide field weaker than the predicted condition $B_{g}<2$, we observe different spreading behavior in the $+z$ and $-z$ directions (bottom row). For the $B_{g}=1.5$ case, there is bi-directional spreading, as observed in the stronger guide field runs, but the spreading is not symmetric about $z=0$. The spreading in the $-z$ direction appears marginally faster than in the $+z$ direction. These differences are further amplified in the $B_{g}=1$ simulation. With no guide field $\left(B_{g}=0\right)$, spreading occurs primarily in the $-z$ direction, with negligible spreading in the $+z$ direction. Since $J_{z}$ is in the $+z$ direction for this reconnection site, the propagation is in the direction of the electron out-of-plane flow, consistent with previous work [Huba \& Rudakov (2002)]. 
To make this quantitative, we measure the spreading speed of the X-line after Hall reconnection begins by finding the length of the X-line in the out-of-plane direction; its time rate of change between an initial and final time is the spreading speed. To do so, we note that the reconnection rate during hyper-resistive reconnection never exceeds 0.01. We observe that when the out-of-plane Hall electric field $E_{H g}=(\mathbf{J} \times \mathbf{B})_{g} /$ nec in a cut in the $y$ direction through the X-line exceeds 0.01, the reconnection has begun its transition to Hall reconnection. We also note empirically that $J_{\max }$ at the time of this transition is always close to 3.3 , which is robust for all the simulations performed here. Thus, we take Hall reconnection as occurring when $J_{\text {max }}$ exceeds a threshold value of $J_{\text {thresh }}=3.3$.

The time frame over which the spreading speed is measured is defined as follows. The initial time $t_{i}$ is defined as the earliest time that $J_{\max }$ exceeds $J_{\text {thresh }}$ over the entire range from $z= \pm 5$. This range of $z$ is chosen because the initial magnetic perturbation that seeds the X-lines is localized in this region, so genuine spreading not being influenced by the growth of reconnection inside the initially perturbed region requires the signal to leave this range in $z$. The final time $t_{f}$ is defined for each simulation as the latest time in the evolution before multiple oblique modes interact; this assessment is done visually by finding where the current develops complicated structure as seen in Fig. 3.7. The length of the X-line at a given time is defined as the extent in $z$ for which $J_{\text {max }}$ exceeds $J_{\text {thresh }}$. The spreading speed is calculated as the difference of the length of the X-line between $t_{f}$ and $t_{i}$ divided by the time difference.

An example of this procedure is presented in Fig. 3.9, where representative 


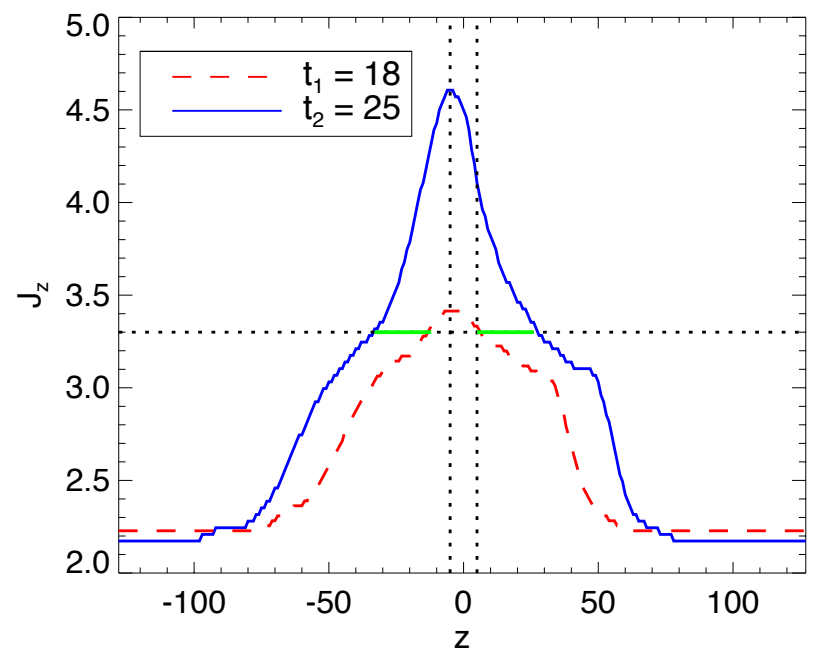

Figure 3.9: Cuts of the stack plot for the $B_{g}=2.5$ case plotted in Fig. 3.8. The dashed (red) line and solid (blue) line are at $t_{i}=18$ and $t_{f}=25$, respectively. The horizontal dotted line is at $J_{\text {thresh }}$ as defined in the text. The vertical dotted lines mark $z= \pm 5$, the approximate extent of the initial magnetic perturbation. The green line denotes the change in length of the X-line between $t_{i}$ and $t_{f}$.

data for the $B_{g}=2.5$ simulation is shown. The initial time is $t_{i}=18$, which is the earliest time that $J_{\max }>J_{\text {thresh }}$ everywhere between $z= \pm 5$, as shown by the red dashed line. The final time is taken to be $t_{f}=25$; a plot of $J_{\max }(z)$ at $t_{f}$ is shown as the blue line. The horizontal dotted line marks the current threshold $J_{\text {thresh }}=3.3$ and the vertical dotted lines mark the boundary of $z= \pm 5$. The change in length between the two times is the distance between the curves at $J_{\text {thresh }}$, marked by the green line segments. The lengths and speeds are calculated separately for the $\pm z$ direction because the speeds in the two directions may be different depending on the strength of the guide field. For the $B_{g}=2.5$ simulation, the change in length in the $+z$ and $-z$ directions are 21 and $20 d_{i 0}$, respectively, and dividing by the time difference gives speeds of $v_{X i}=3.0 c_{A 0}$ for the speed in the $+z$ direction (the direction of ion out-of-plane flow) and $v_{X e}=2.9 c_{A 0}$ for the speed in the $-z$ direction 


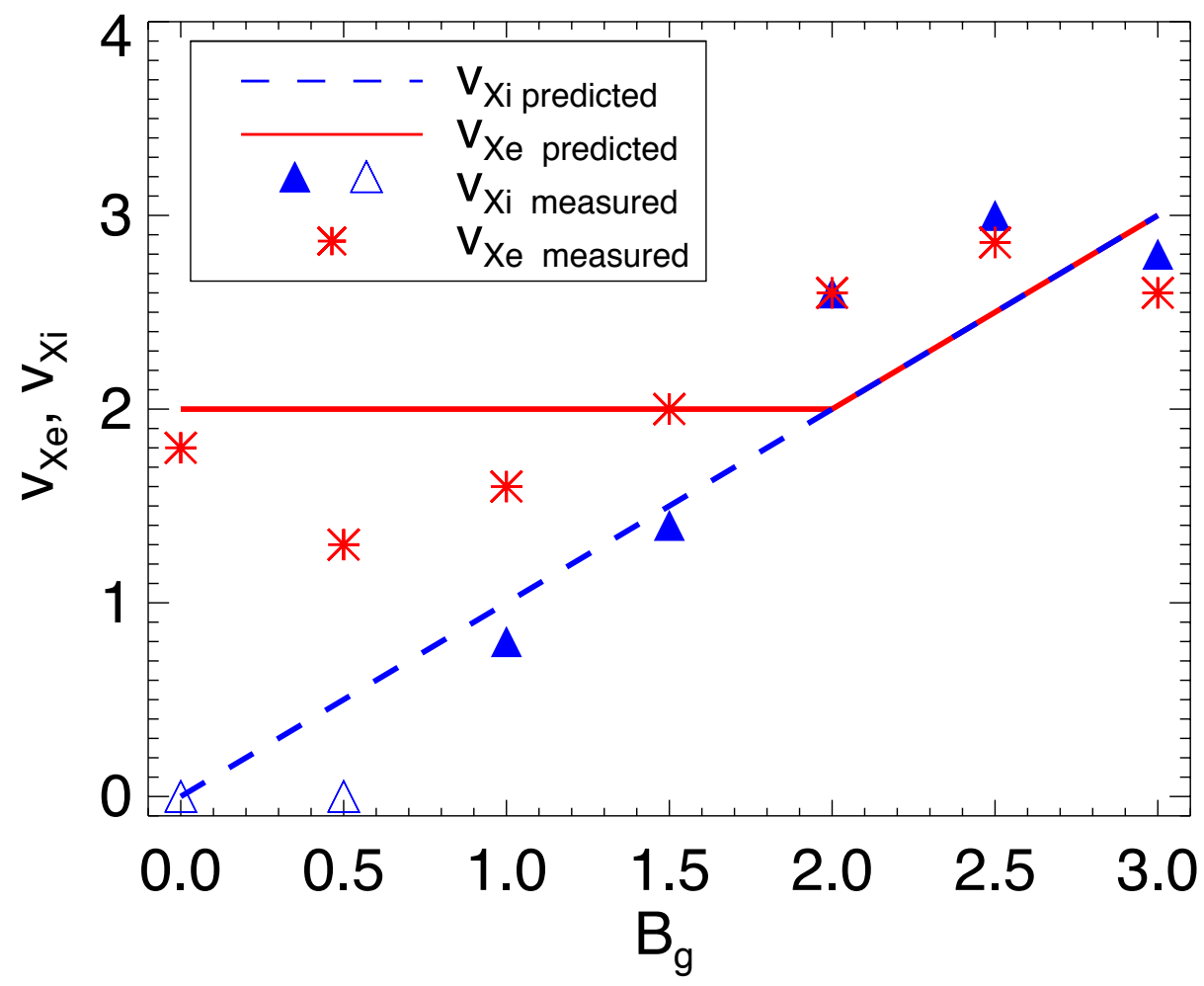

Figure 3.10: Spreading speeds $v_{X i}$ and $v_{X e}$ as a function of guide field $B_{g}$. The red asterisks are the measured values of $v_{X e}$; the solid red line is the prediction from Eq. (3.3). The solid blue triangles are the measured values of $v_{X i}$; the open triangles are for simulations for which no spreading was measured. The dashed blue line is the prediction from Eq. (3.6).

(the direction of electron out-of-plane flow).

The initial and final times $t_{i}$ and $t_{f}$ for each simulation are illustrated in Fig. 3.8 as the vertical dotted green lines. The dashed white lines connect the extent of the X-line at the initial and final times. By inspection, one can see that the technique we employ to measure the extent of the X-line appropriately captures the evolution of the X-line length. Also, since the region of stronger current is rather straight between the beginning and final times, this implies the spreading speed is approximately constant in time. 
The measured X-line spreading speeds $v_{X e}$ and $v_{X i}$ are calculated as the time rate of change of the length of the $\mathrm{X}$-line, which is equivalent to the slope of the white dashed lines in Fig. 3.8. The results for the spreading speed in both directions are plotted as a function of guide field $B_{g}$ in Fig. 3.10. The measured value of the spreading speed is given by the solid blue triangles for $v_{X i}$ and the red stars for $v_{X e}$. Note, $v_{X i}$ for $B_{g}=0.0$ and 0.5 is plotted as zero as the hollow blue triangles. This is because the Hall reconnection signal is found to not extend past $z= \pm 5$ for either simulation during the time considered.

To compare these results to the theory, note that the electrons carry all of the out-of-plane current in the $-z$ direction in our simulations. Therefore, in the weak guide field regime $B_{g}<2$, Eq. (3.3) predicts that the spreading speed in the direction of the electron current $v_{X e}$ is the speed of the electrons given in Eq. (3.1), which is independent of $B_{g}$. When $B_{g} \geq 2$, the spreading speed is determined by the Alfvén speed given by Eq. (3.2), which increases linearly with $B_{g}$. The predicted speed of X-line spreading in the direction of the ion current $v_{X i}$ is the Alfvén speed due to the guide field, as given by Eq. (3.6), which increases linearly with $B_{g}$ for all guide field strengths.

The predicted spreading speeds $v_{X e}$ and $v_{X i}$ are depicted in Fig. 3.10 by the solid red line and the dashed blue line, respectively. Qualitatively, the data reveal that the nature of X-line spreading is sensitive to the strength of the guide field. To interpret this more quantitatively, we first discuss the estimated uncertainties in our speed measurements. If we use a higher value of the current threshold $J_{\text {thresh }}$, the spreading speed changes on the order of $15-20 \%$, which we take as the uncertainty. 
We note that for the large guide field runs $B_{g} \geq 2$, the speeds in either direction are within the uncertainties of each other. However, for $B_{g}<2$, the speeds in either direction are separated by more than their uncertainty. These two results suggest that the spreading mechanism is the same in both directions for $B_{g} \geq 2$ and is different in either direction for $B_{g}<2$, which quantitatively agrees with Eq. (3.11).

For the absolute spreading speeds, when the estimated uncertainties are taken into account, the measured values agree pretty well with the predicted speeds. It is unexpected that the $B_{g}=3$ speeds are slower than $B_{g}=2.5$, but both are within the uncertainties of the predicted value. Also, it is expected that for $B_{g}=0.5$, a non-zero value could be obtained if there had been a longer time before the oblique modes started interacting. Therefore, we conclude that data in Fig. 3.10 quantitatively support the theory presented in Sec. 3.2.

In summary, the mechanism of X-line spreading in the out-of-plane direction is qualitatively different depending on the strength of the guide magnetic field. For $B_{g} \geq B_{\text {crit }, e}, \mathrm{X}$-line spreading occurs bi-directionally along the guide field at the Alfvén speed. For $B_{g} \leq B_{\text {crit,e }}$, X-line spreading occurs bi-directionally along the guide field, but the spreading speed in the direction of the current carriers is the speed of the current carriers and in the direction opposite of the primary current carriers the spreading speed is the Alfvén speed. Measurements of X-line spreading for the hyper-resistive reconnection that precedes Hall reconnection agree with the results obtained from measuring the Hall reconnection spreading (not shown). Therefore, the main result of this study applies both to Hall and hyper-resistive reconnection in a two-fluid model. 


\subsection{Summary}

In summary, the mechanism of X-line spreading in the out-of-plane direction is qualitatively different for strong guide magnetic fields than it is for weak guide fields. For weak guide fields, the reconnection signal is propagated by the current carriers, as has previously been established; for strong guide fields, the reconnection signal is propagated by Alfvén waves along the guide field. In general, the spreading speed in either out-of-plane direction is given by the maximum of the speed of the current carriers in that direction and the Alfvén speed based on the guide field, as given by Eq. (3.9).

Because the changeover from one spreading mechanism to the other is abrupt, there is a critical guide field strength (for each direction) at which the nature of the spreading switches. This critical field depends only on the strength of the reconnecting magnetic field, the ion inertial scale, the thickness of the electron dissipation region, and the fraction of the current carried by each species, as given by Eq. (3.10). When the guide field $B_{g}$ exceeds the critical field, the spreading is due to Alfvén waves; when it is smaller, the spreading is due to the current carriers. The weak guide field result is consistent with previous numerical work of X-line spreading [Huba \& Rudakov (2002); Shay et al. (2003); Karimabadi et al. (2004); Lapenta et al. (2006); Nakamura et al. (2012)], but the new result generalizes the predictions to include a guide field.

The present results may be relevant for interpreting observations of reconnection in many settings. For example, in laboratory experiments, X-line spreading has 
been observed to be bi-directional and at the Alfvén speed in the strong guide field limit [Katz et al. (2010)] and uni-directional in the small guide field limit [Dorfman (2012)]. These results are consistent with the results of the present study.

Another potential application is for solar flares. Two-ribbon flare evolution is marked by the ribbons moving apart from each other as time evolves, which is interpreted as newly reconnected field lines piling on top of previously reconnected field lines. In addition to this behavior, bi-directional spreading or elongation of the ribbon in the direction parallel to the ribbons along the polarity inversion line has been observed [Qiu (2009)]. It was shown that the spreading speed was consistent with the Alfvén speed [Qiu (2009)]. Since the reconnection driving the flare is most likely to have a sizable guide field, the present results suggest that this type of bi-directional spreading at the Alfvén speed would be expected.

The Bastille Day flare exhibits this spreading, as well [Qiu et al. (2010)]. From geometrical considerations of the magnetic fields of the flare loops, it was argued that the guide field was of comparable size as the reconnecting field, with $B_{g} \simeq 0.4-1.2$ times the reconnecting field [Qiu et al. (2010)]. We can check this using the present results and the observed properties of the spreading. From the observations, the spreading speed ranged between $30-70 \mathrm{~km} / \mathrm{s}$ [Qiu et al. (2010)]. Let us assume the spreading is governed by Alfvén waves. Assuming an average density of $n=10^{13} \mathrm{~cm}^{-3}$ [Qiu et al. (2010)], the guide field ranges from $B_{g} \simeq 15-100$ G using Eq. (3.2). The motion of the ribbons normal to the ribbons was $20 \mathrm{~km} / \mathrm{s}$ [Qiu et al. (2010)], which is expected to be correlated to the inflow speed at the reconnection site. Since the inflow speed is often taken to be 0.1 of the Alfvén speed 
based on the reconnecting field, the reconnecting field strength $B_{r e c} \simeq 140 \mathrm{G}$. These results suggest the guide field is about $0.1-0.7$ of the reconnecting field. Despite the large uncertainties, the two techniques give similar results. This analysis is obviously oversimplified and merely presented as an example of how the results can be used, but it is hoped that future work will allow for a meaningful assessment of the relative strengths of the guide and reconnecting fields. The reason this may be useful, as emphasized by [Qiu et al. (2010)], is that the strength of the guide field is known to influence the production of secondary islands [Drake et al. (2006)], and it has been suggested that the presence of secondary islands (plasmoids) is important for particle acceleration [Drake et al. (2006)]. 


\section{Chapter 4}

\section{Structure of Reconnection Exhaust}

The previous chapter addressed guide field reconnection and explored the basic nature of X-line spreading. In nature, it is not difficult to imagine reconnection being limited to a particular region (e.g., by the geometry of the current sheet). This type of reconnection will be referred to as localized reconnection (i.e., not spreading). In this chapter, we study localized reconnection by using an anomalous resistivity. What differences exist, if any, between localized reconnection and spreading reconnection?

This chapter addresses localized magnetic reconnection with and without a guide field. Section 4.1 discusses previous work and observations where localized reconnection may play a role. Theoretical predictions of the behavior of localized reconnection are discussed in Section 4.2. In Section 4.3, the setup for simulations that are used to test our predictions are discussed. The results for the simulations are presented in Section 4.4. Applications in the solar wind and corona are discussed in Section 4.5.

\subsection{Introduction}

In the solar wind, magnetic reconnection outflows, or exhausts, measuring

$600 R_{E}\left(3.8 \times 10^{6} \mathrm{~km}\right)$ [Gosling et al. $\left.(2007)\right]$ and $390 R_{E}\left(2.5 \times 10^{6} \mathrm{~km}\right)[$ Phan 
a)

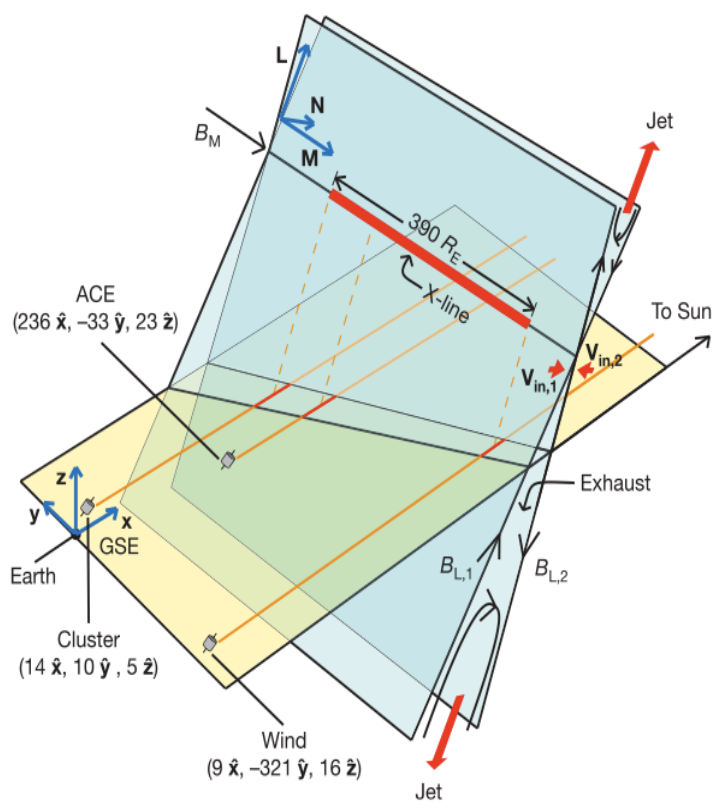

b)

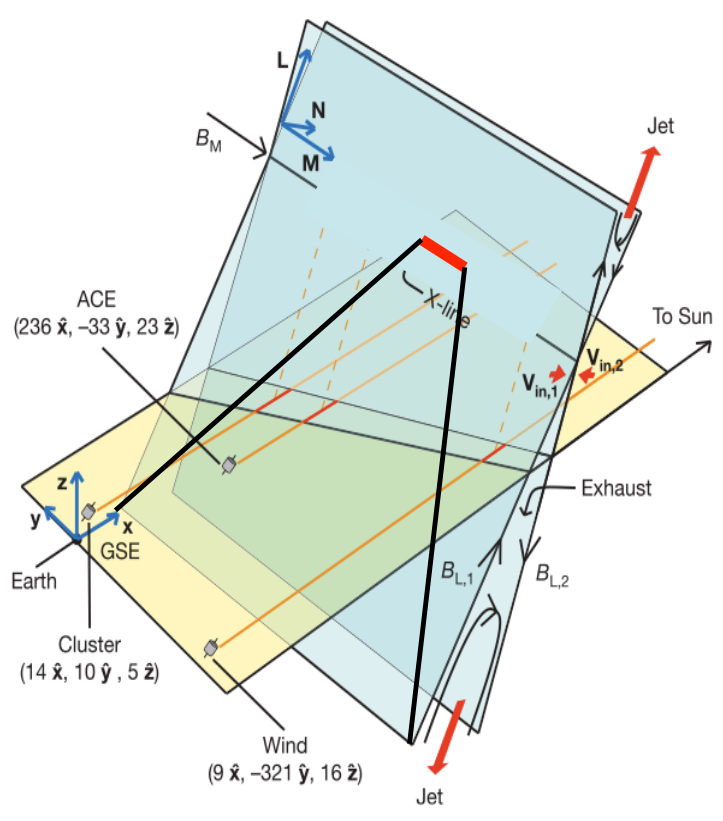

Figure 4.1: (a) Diagram of a large-scale reconnection site that accounts for the total length of the reconnection outflow, or exhaust. Reprinted with permission from Phan et al. (2006). (c) 2006 by Nature Publishing Group. (b) An alternate scenario where the reconnection site is localized in the out-of-plane direction, and the exhaust expands into the out-of-plane direction to create the extended exhaust signature. Adapted from Phan et al. (2006). 
et al. (2006)] in length have been observed. A schematic of the Phan et al. (2006) event is detailed in Fig. 4.1(a), where three satellites (ACE, Cluster, and Wind separated by a large distance) measured the same reconnection signature within a short period of time. The authors assumed that the extended exhaust was caused by an extended X-line. If this is the case, what mechanism is responsible for these large scale structures? It was suggested in Phan et al. (2006), the large exhaust could be formed by a small X-line forming near the sun and spreading as the X-line convects away from the sun. This configuration is depicted in Fig. 4.1(a), where the $\mathrm{X}$-line is symbolized by the solid red bar and the edges of the reconnection exhaust are marked by the shaded blue planes which extend from the X-line. However, the satellites only observed the exhausts, so it is not obvious that the $\mathrm{X}$-line is necessarily as extended as the exhausts. Another explanation that exists is the $\mathrm{X}$-line is localized in a small region and the exhaust extends into the out-of-plane direction. This configuration is depicted in Fig. 4.1(b), where the X-line is marked by the much shorter solid red bar and the edges of the exhaust are marked by the solid black lines that extend from the X-line.

Localized reconnection may play a role in the creation of supra-arcade downflows (SADs); because of their sinusoidal nature they are often referred to as "tadpoles". SADs are dark features that appear at the top of coronal arcades. These features descend towards the sun during solar flares [McKenzie \& Hudson (1999); McKenzie (2000)]. A TRACE image of SADs can be seen in Fig. 4.2, indicated by the black arrows. It was suggested in Cassak et al. (2013), that localized reconnection is important for the creation of SADs, because localized reconnection can 


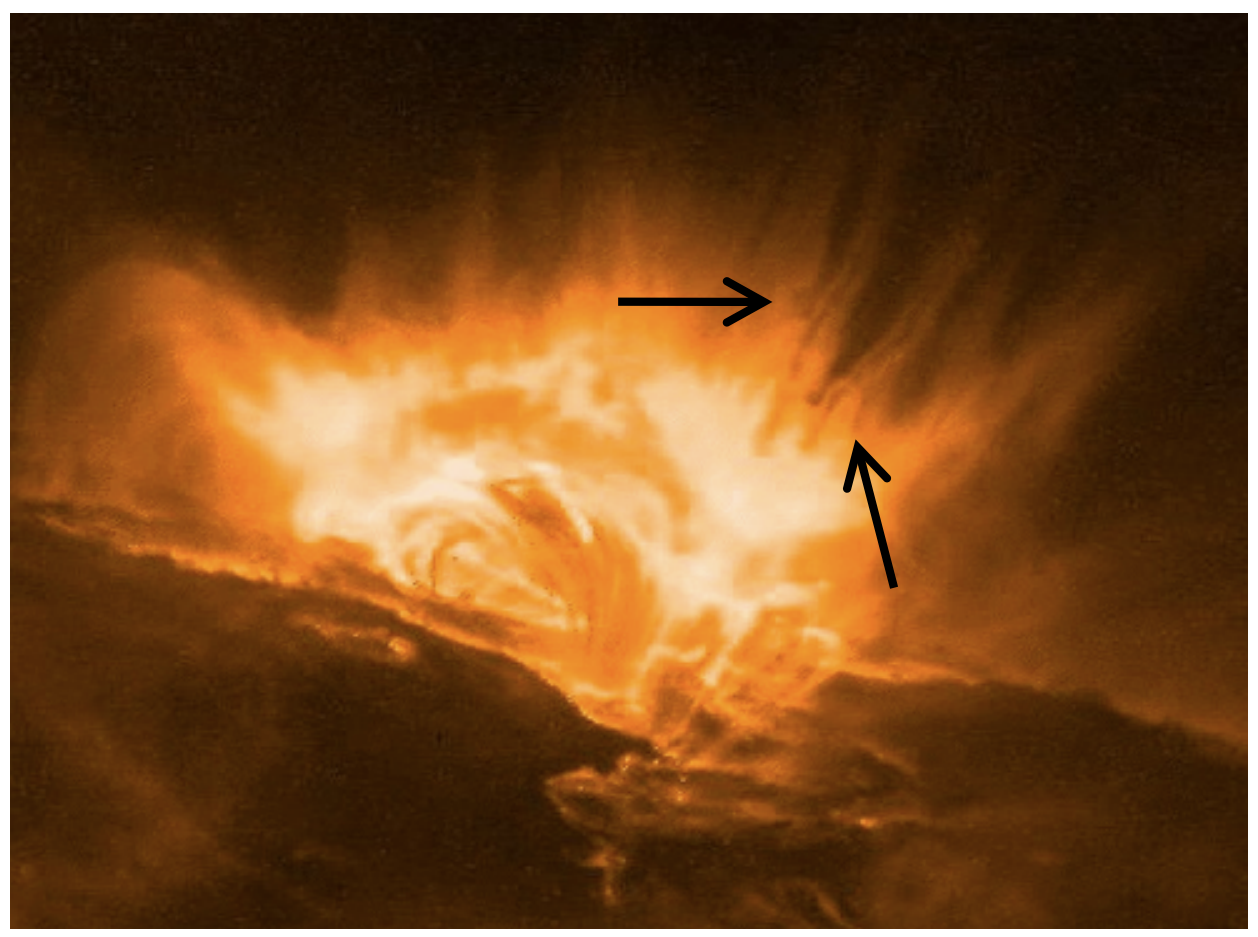

Figure 4.2: TRACE image of supra-arcade downflows (SADs) taken on April 21, 2002. The SADs are marked by the black arrows. Image credit: NASA/TRACE

create a collimated reconnection exhaust, which is important for carving out the thin SADs. Applications to SADs will be discussed later. Some of the work presented in this chapter is in preparation to be submitted for publication [Shepherd et al. (2014)], while some of this work was published in The Astrophysical Journal Letters [Cassak et al. (2013)].

\subsection{Theory}

Here, we develop the physical characteristics and predictions of the structure of localized reconnection as a function of guide field and length of the X-line. To do so, we treat a quasi-two-dimensional system, meaning the equilibrium parameters do not depend strongly on the direction normal to the reconnection plane over time. 
We assume the plasma parameters are symmetric on either side of the current layer; asymmetries [Cassak \& Shay (2007)] are not considered here.

We begin by examining two-dimensional magnetic reconnection. Figure 4.3 displays a sketch of a typical collisionless two-dimensional X-line. The purple lines represent the magnetic fields above the current sheet, while the orange lines represent the fields below the current sheet. The black squares represent the guide field piercing the $x y$ plane. When reconnection begins, the magnetic fields are pulled towards the neutral line where they break and reconnect at the ' $\mathrm{X}$ ' point. The bent magnetic field lines caused by the magnetic reconnection process generate a rotational discontinuity $(\mathrm{RD})$ that propagates along the reconnected magnetic field line, represented by the blue line. There is an inflow $\left(v_{i y}\right)$ across the boundary, that is then converted into reconnection outflow $\left(v_{i x}\right)$. This inflow is required for mass conservation to be satisfied, because the outflow continually expands into the inflow direction.

How does this picture change when we consider a localized X-line? A recent paper by Sasunov et al. (2012) addressed localized reconnection through a theoretical approach to develop profiles for specific plasma parameters and compare these profiles for magnetic field, plasma flows, temperature and density to observations of reconnection in the solar wind. The predicted geometry of the magnetic reconnection exhaust is sketched in Fig. 4.4(a). The X-line is localized in the out-of-plane (M) direction. The dotted lines that extend from the X-line are the last field lines that reconnect at the edge of the X-line. Figure 4.4(b) is a view of the reconnection exhaust at the location of the $W$ plane located in the first image. The exhaust is 

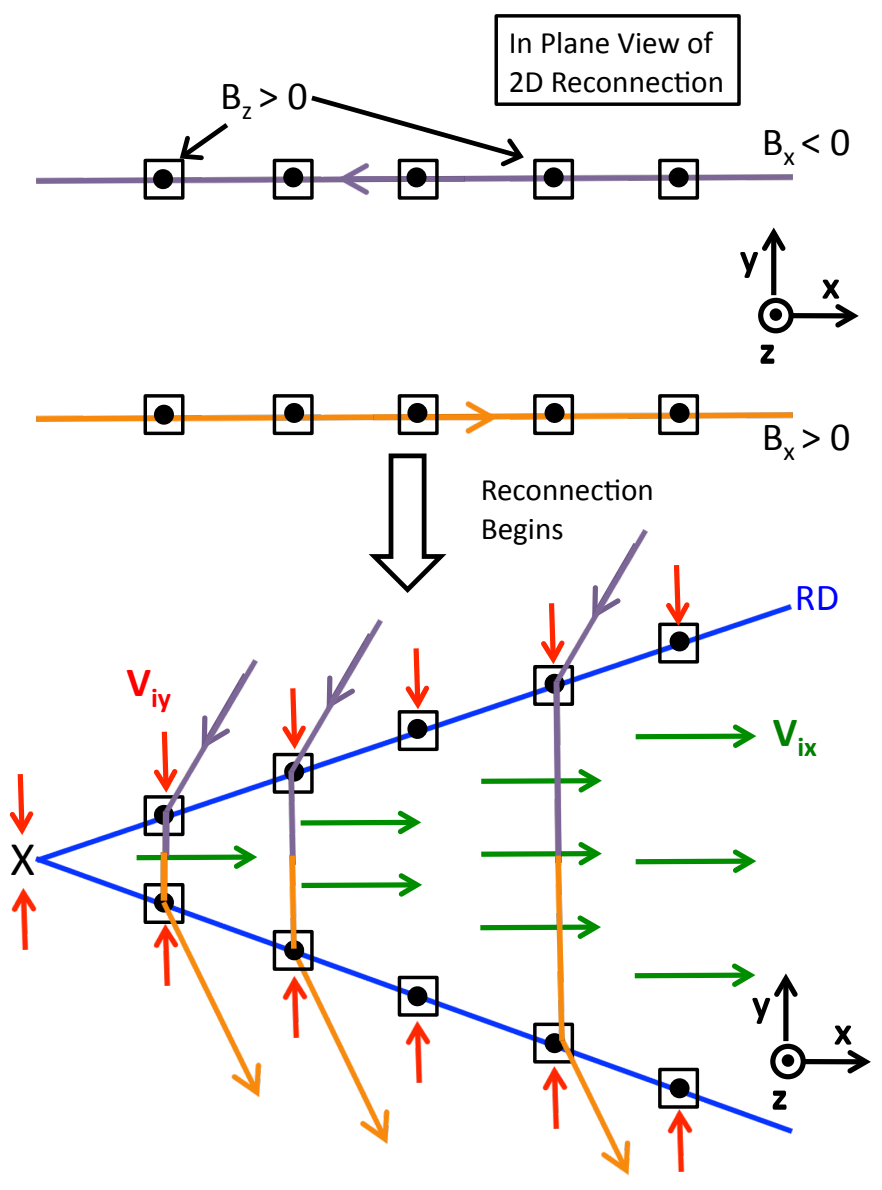

Figure 4.3: Sketch of a two-dimensional reconnection site. The red ion inflows $V_{i y}$ flow through a rotational discontinuity and are turned into the green outflows $V_{i x}$. The exhaust will expand continually into the inflow direction for $2 \mathrm{D}$ reconnection. 
viewed downstream looking back towards the X-line. The inflow direction is the vertical axis and the out-of-plane direction is along the horizontal axis. The thick black lines mark the tangential discontinuities and form primarily the upper and lower boundaries of the exhaust. The red lines are rotational discontinuities formed by the reconnection process and compose the side boundaries of the exhaust. A contact discontinuity is predicted to occur at the center of the exhaust. A contact discontinuity separates two different plasma populations that are in pressure balance; because there is pressure balance, there is no force between the two populations and they will not mix in the MHD model. In reality, this discontinuity would not exist because the two populations will naturally mix.

These sketch qualitatively provide the structure of localized reconnection. We now derive quantitative features of localized reconnection by geometry of the magnetic field. A sketch of the magnetic field geometry from the inflow direction is shown in Fig. 4.5. The localized reconnection X-line of length $2 W_{0 z}$ is marked by the dashed line. The purple and orange lines mark the last field lines to be reconnected at the edge of the X-line. In the diagram on the left, the purple lines represent the magnetic fields above the current sheet, while the orange lines represent the fields below the current sheet. During reconnection, the magnetic field lines break, reconnect, and then retreat from the X-line, as seen in the sketch on the right. This sketch reveals that the exhaust extends into the out-of-plane direction; the amount it does is determined by the opening angle $\theta$. From the diagram, we see 

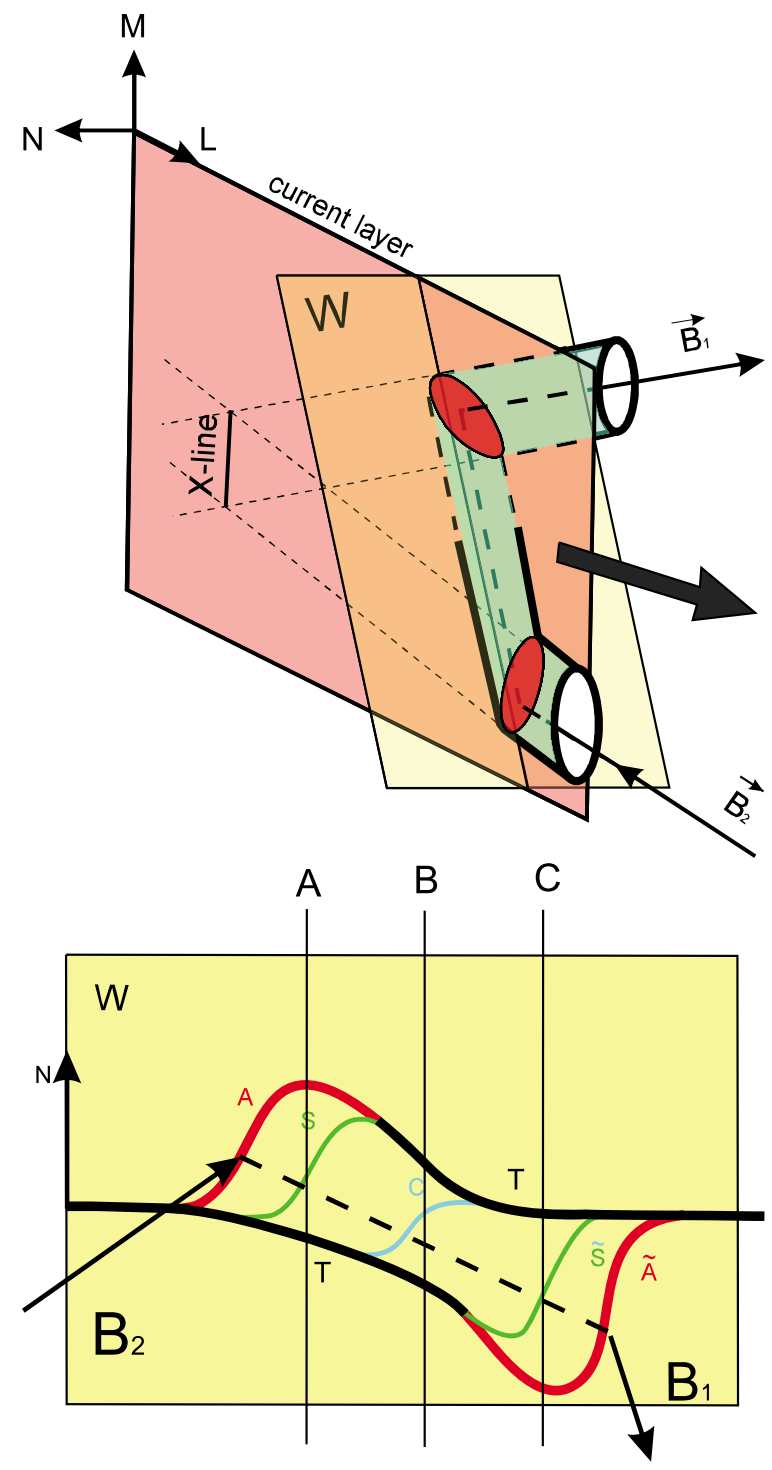

Satellite A

Satellite B

Satellite C

Figure 4.4: The top panel displays the 3D geometry of the magnetic field. The coordinate system for this figure is as follows, the outflow direction is L, the inflow direction is $\mathrm{N}$, and the out-of-plane direction is $\mathrm{M}$. The dashed lines represent the last reconnected field lines. The $W$ plane marks the position of the cut in the bottom panel. It is predicted that the 3D exhaust structure will consist of rotational discontinuities (red), a slow shocks (green), a contact discontinuity (blue), and tangential discontinuities (black). Reprinted with permission from Sasunov et al. (2012). (c) 2012 by the American Geophysical Union. 

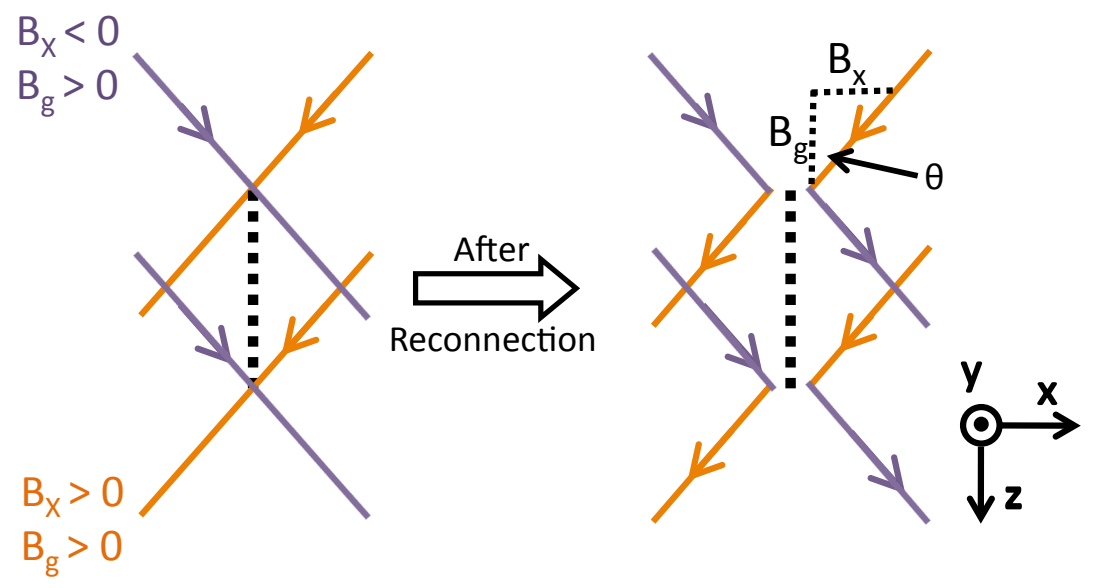

Figure 4.5: A sketch of the 3D reconnection geometry. The purple lines represent the magnetic fields above the current sheet, while the orange lines represent the fields below the current sheet. After reconnection begins, the reconnection exhaust forms a cone-like structure into the out-of-plane direction with opening angle $\theta=$ $\tan ^{-1}\left(B_{x} / B_{z}\right)$.

the opening angle is controlled by the strength of the guide field $B_{g}$,

$$
\theta_{\text {pred }}=\tan ^{-1}\left(\frac{B_{x}}{B_{g}}\right)
$$

where $B_{x}$ is the upstream asymptotic value of the reconnecting magnetic field.

The localized reconnection X-line of length $2 W_{0 z}$ is marked by the dashed line. The purple lines represent the magnetic fields above the current sheet, while the orange lines represent the fields below the current sheet. During reconnection, the magnetic field lines break and reconnect and then retreat from the X-line. The colored magnetic field lines in this sketch are the reconnected field lines at the edge of the reconnection region. As in the $2 \mathrm{D}$ picture Fig. 4.3, an $\mathrm{RD}$ propagates along each reconnected magnetic field line as well as all areas in between the ends of the reconnection region. Therefore, in 3D localized reconnection, the $\mathrm{RD}$ is localized in 


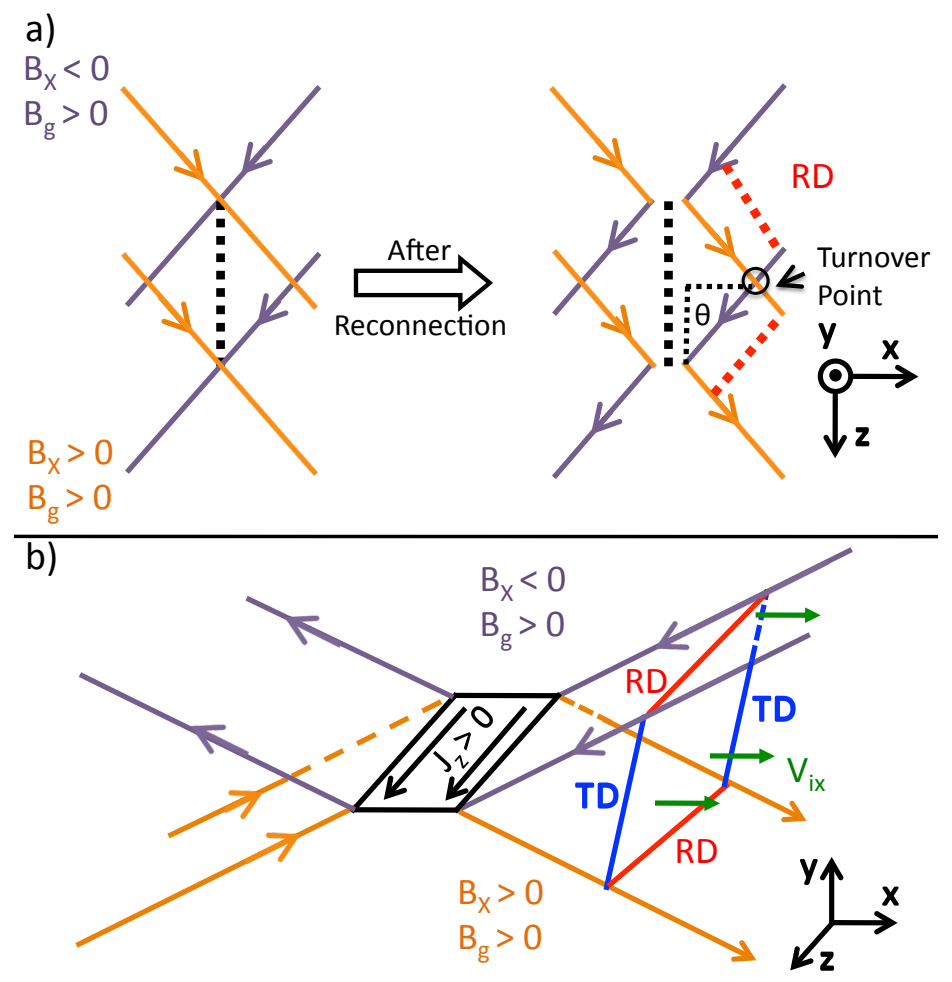

Figure 4.6: (a) Sketch of the magnetic field geometry in the $x z$-plane. Rotational discontinuities propagate in a ribbon in the out-of-plane direction. (b) Sketch of the 3D structure of the reconnection exhaust. The exhaust forms a parallelogram structure, where each side of the parallel is either a TD or an RD.

a ribbon (shown as the red dashed line in Fig. 4.6(a)). Two RD ribbons propagate above and below the current sheet in the out-of-plane direction. At tangential discontinuity (TD) is formed at the boundary of the reconnected magnetic field lines and the magnetic fields that do not reconnect. The ribbon structure of the exhaust can be seen more explicitly in the 3D sketch in Fig. 4.6(b), where the blue marks the location of TDs and the red lines mark the location where RDs are located. We can now see that the reconnection exhaust has a distinct parallelogram shape, with opposite sides being either an RD or a TD as in Fig. 4.4.

In three-dimensional reconnection, the reconnection exhaust can collimate af- 
ter a short distance from the X-line. We call the location where the collimation begins the "turnover point". Collimation begins when the reconnection exhaust stops expanding in the $y$ (inflow) direction. Using the magnetic field geometry we can predict the location of the turnover point. As is shown in Fig. 4.6, the reconnected magnetic fields (or RDs) cross the $z=0$ reconnection plane at the turnover point indicated in Fig. 4.6(a). From Eq. 4.1, the magnetic field makes an angle $\theta$ with the $z$-axis, such that $\tan (\theta)=B_{x} / B_{g}$, where $B_{x}$ is the reconnecting magnetic field. Also, $\theta$ defines the angle between the turnover point distance and half-length of the $\mathrm{X}$-line, $\tan (\theta)=l_{x} / l_{z}$. Solving for the distance to the turnover point $l_{x}$, we find the distance to the turnover point to be

$$
l_{x}=l_{z} \frac{B_{x}}{B_{g}} .
$$

The turnover point is indicated as the black circle in Fig. 4.6(a), where the last reconnected field line passes through the $z=0$ plane.

We can predict the thickness of the reconnection exhaust at the turnover point. The expected thickness of the collimated exhaust was derived in Cassak et al. (2013). Consider a recently reconnected flux tube with flux $\Delta \Phi$ formed by reconnection at an X-line of finite extent $l_{z}$ in the out-of-plane direction $z$ and length $\Delta l_{x}$ in the outflow direction $x$. Just downstream of the X-line,

$$
\Delta \Phi \sim B_{y} l_{z} \Delta l_{x}
$$


where $B_{y}$ is the reconnected (normal) component of the magnetic field. After the flux tube has convected downstream, the magnetic field in the exhaust is predominantly in the $z$ direction, so the flux is

$$
\Delta \Phi^{\prime}=B l_{y}^{\prime} \Delta l_{x}
$$

where $l_{y}$ is the width of the flux tube in the $y$ direction, $B=|\boldsymbol{B}|$ is the magnitude of the total magnetic field, and the prime denotes post-convection downstream. By conservation of flux, $\Delta \Phi=\Delta \Phi^{\prime}$, so solving Equations (4.3) and (4.4) for $l_{y}^{\prime}$ gives

$$
l_{y}^{\prime} \sim l_{z} \frac{B_{y}}{B}
$$

Regardless of whether reconnection is $2 \mathrm{D}$ or $3 \mathrm{D}$, the maximum value of $B_{y}$ is approximately 0.1 of the reconnecting field $B_{x}$ since the normalized rate of fast reconnection is close to 0.1 [Cassak et al. (2013)].

We can also use a geometric argument to predict the thickness of the reconnection exhaust at the turnover point. This is illustrated in Fig. 4.7 with the collimation of the exhaust beginning at the turnover point located at $l_{x}$ and the thickness of the exhaust at the turnover point is given as $l_{y}$. The angle $\theta$ is a measure of the angle between $B_{y}$ and $B_{x}$ and between $l_{y}$ and $l_{x}$. If we use this geometry, we find that $\tan \theta=B_{y} / B_{x}=l_{y} / l_{x}$, therefore $l_{y}=l_{x} B_{y} / B_{x}$. We know that $l_{x}=l_{z} B_{x} / B_{g}$, from 


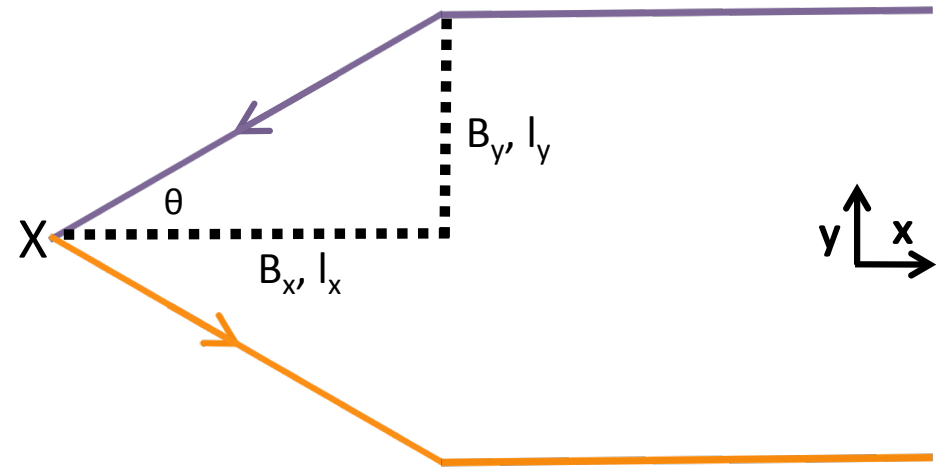

Figure 4.7: Sketch of the $x y$ magnetic reconnection geometry. The magnetic field opens up into the inflow direction with an angle $\theta$, where $\tan \theta=B_{y} / B_{x}=T_{E} / D_{x}$.

Eq. 4.2. Solving for the exhaust thickness yields

$$
l_{y}=l_{z} \frac{B_{y}}{B_{g}}
$$

This is similar to the argument provided by Cassak et al. (2013), except only the guide field contributes to the denominator instead of the total magnitude of $\boldsymbol{B}$.

\subsection{Simulation Setup}

To test the predictions on X-line structure, three-dimensional numerical simulations are performed using the two-fluid code F3D [Shay et al. (2004)]. The code updates the continuity, momentum, and induction equations with the Ohm's law including electron inertia. The Hall term is turned off for these simulations. Magnetic fields and densities are normalized to arbitrary values $B_{0}$ and $n_{0}$. Velocities are normalized to the Alfvén speed $c_{A 0}=B_{0} /\left(4 \pi m_{i} n_{0}\right)^{1 / 2}$. Lengths are normalized to an arbitrary length $L_{0}$. Times are normalized to $L_{0} / c_{A 0}$, electric fields to 
$E_{0}=c_{A 0} B_{0} / c$, and temperatures to $T_{0}=m_{i} c_{A 0}^{2}$.

Magnetic reconnection is initiated and localized in the out-of-plane direction by utilizing anomalous resistivity $\left(\eta_{\text {anom }}\right)$. Anomalous resistivity is discussed in more detail in Chapter 1. Anomalous resistivity achieves fast reconnection and allows us to fix the X-line length in the out-of-plane direction. The extent of the X-line is dependent on the profile of $\eta_{\text {anom }}$, and the reconnection does not spread. Anomalous resistivity has the form

$$
\eta_{\text {anom }}=\eta_{0} e^{-(x / 0.5)^{2}-(y / 0.5)^{2}}\left[\frac{\tanh \left(z+w_{0 z}\right)-\tanh \left(z-w_{0 z}\right)}{2}\right],
$$

where $\eta_{0}=0.01$ and $w_{0 z}$ sets the half-width of the X-line length in the out-of-plane direction.

Simulations are performed in a three-dimensional domain of size $L_{x} \times L_{y} \times L_{z}=$ $51.2 \times 25.6 \times 256.0$ in arbitrary units, where $x$ is the direction of the oppositely directed field, $y$ corresponds to the inflow direction if the simulations were twodimensional, and $z$ is the out-of-plane direction. The plasma is assumed to be isothermal. Boundaries in the $x, y$, and $z$ directions are periodic, but the system is long enough in the $z$ direction that the periodic boundaries do not affect the dynamics on the time scales of import to the present study. A grid scale of $\Delta x \times$ $\Delta y \times \Delta z=0.05 \times 0.05 \times 1.0$ in arbitrary units. Using a stretched grid in the outof-plane direction has been done before [Shay et al. (2003)], and is acceptable since the in-plane dynamics are on smaller scales than the out-of-plane dynamics. All equations employ a fourth-order diffusion with coefficient $D_{4 x}=D_{4 y}=2.5 \times 10^{-5}$ 
in the $x$ and $y$ directions. In the out-of-plane direction the fourth-order diffusion coefficient $D_{4 g}$ depends on the speeds in the out-of-plane direction. For $B_{g}=0.0$ the fourth-order diffusion coefficient is $D_{4 g}=0.064$ and for 3.0 the fourth-order diffusion coefficient is $D_{4 g}=$ and 0.097 , respectively. The values of $D_{4 g}$ were tested by varying the value by a factor of two to ensure that $D_{4 g}$ does not play a significant role in the dynamics.

The initial magnetic field configuration is a double tearing mode setup where

$$
B_{x}(y)=B_{0}\left[\tanh \left(\frac{y+L_{y} / 4}{W_{0}}\right)-\tanh \left(\frac{y-L_{y} / 4}{W_{0}}\right)-1\right]
$$

where $B_{0}=1$ is the asymptotic value of the magnetic field and $W_{0}=0.2$ is the initial half-width of the initial current sheet in the inflow direction. We utilize a constant and uniform temperature $T=1.0$. Total pressure is balanced by non-uniform gas pressure. The system parameters are the same used in chapter 3, except for the exclusion of the Hall term.

\subsection{Results}

We show the primary difference between the exhaust structure of localized reconnection and spreading reconnection in Fig. 4.8 in the strong guide field regime, $B_{g}=3.0$. Figure 4.8 displays the reconnection exhaust $v_{i x}$ in the $x z$ plane in a cut made through the $\mathrm{X}$-line. The white ion flow represents a flow with a positive velocity, and black ion flow represents a flow with negative velocity. Reconnection for a system containing the Hall term is shown in panel Fig. 4.8(a). The square 
a)

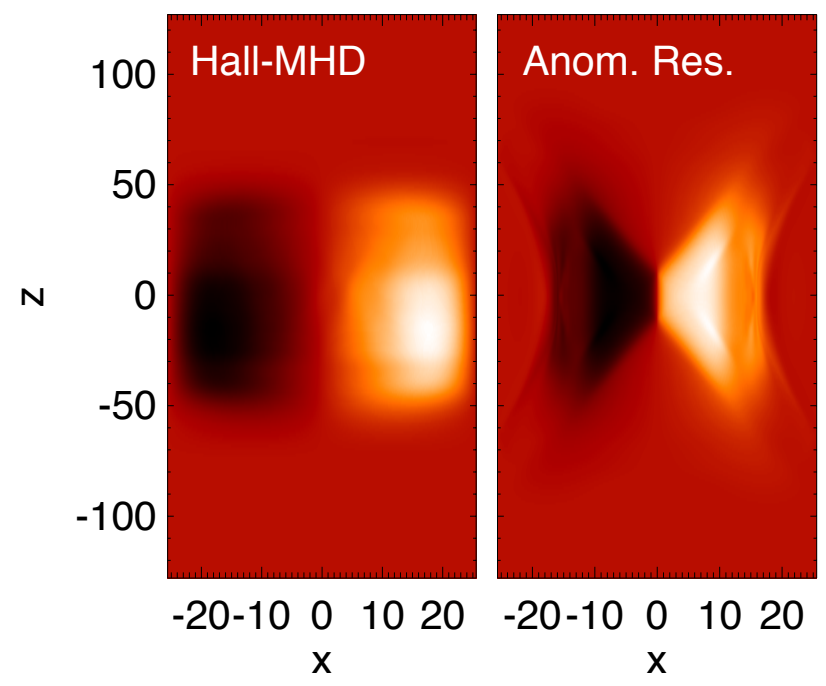

Figure 4.8: (a) Image of the exhaust in $x z$ plane due to Hall reconnection. (b) Image of the reconnection exhaust due to anomalous resistivity.

shape of the exhaust is attributed to the reconnection spreading in the out-of-plane direction. For magnetic reconnection with an anomalous resistivity, the anomalous resistivity is localized between $z= \pm 10\left(w_{0 z}=10.0\right)$. As we see in Fig. $4.8(\mathrm{~b})$, the magnetic reconnection remains localized to $z= \pm 10$ and the reconnection exhaust expands into the out-of-plane direction forming a cone-like shape. An important item of note is that an exhaust of any length could be created with or without spreading because of the presence of a guide field.

The cone-like shape of the reconnection exhaust is controlled by the magnetic field geometry, as predicted by Eq. 4.1. Images of the reconnection exhaust as a function of guide field in Fig. 4.9. As can be seen, the larger the guide field the more the reconnection extends into the out-of-plane direction. We measure the opening angle by finding the boundary of the exhaust beyond the initial reconnection region 


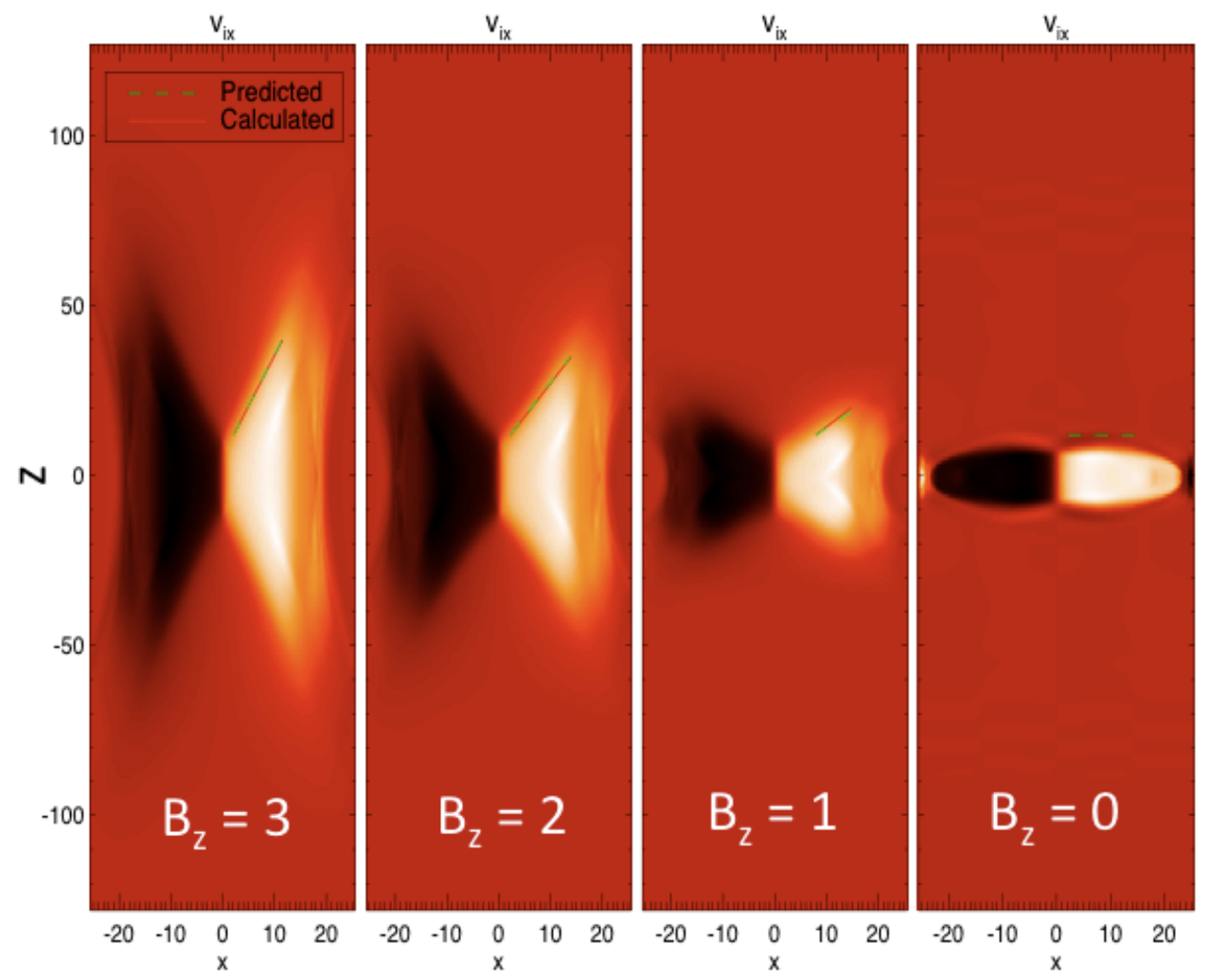

Figure 4.9: Plots of the reconnection exhaust in the $x z$ plane for $B_{g}=(3,2,1,0) B_{0}$.

$z>10$. The boundary is chosen by taking cuts of the exhaust $v_{i x}$ in the outflow direction and the edge is chosen to be where $v_{i x}=1.0$. The measured angle is defined as $\theta_{\text {measured }}=\tan ^{-1}\left(L_{z b} / L_{x b}\right)$, where $L_{z b}$ and $L_{x b}$ are the lengths of the boundary in the out-of-plane direction and outflow direction, respectively. The predicted (solid line) and measured (triangles) opening angle $\theta$ as a function of guide field $B_{g}$ are shown for each simulation in Fig. 4.10. The measured angle agrees very well with the predicted opening angle given by Eq. 4.1.

In the plane of reconnection $(x y)$, we find the reconnection exhaust becomes collimated in the inflow direction. We show the collimation effect of localized reconnection with varying guide field in Fig. 4.11, where each image is of the reconnection 


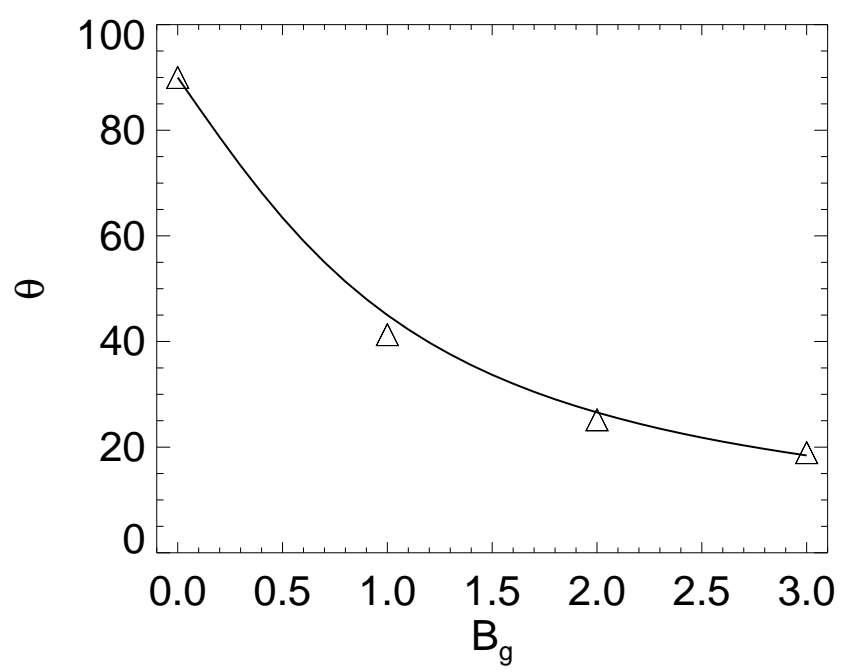

Figure 4.10: Plot of opening angle $\theta$ versus guide field $B_{g}$. The predicted opening angle given as $\theta_{\text {predicted }}=\tan ^{-1} B_{x} / B_{z}$ is marked by the solid line. The measured angle is marked by the triangles. The measured opening angle agrees well with the predicted opening angle.

exhaust $v_{i x}$. The inflow and outflow directions are the horizontal and vertical axes, respectively. Each of these simulations have the same $w_{0 z}=10.0$ parameter. For reference, vertical dashed lines are added a distance 0.5 in arbitrary units from the center of the exhaust. We see for $B_{g}=3.0,2.0$, and 1.0, the exhaust expands and then collimates (runs parallel to the $x$ direction) at the turnover point located at $x \sim 4,7$, and 10, respectively. According to Eq. 4.2, the turnover point will be $x \sim 3.3,5$, and 10 for $B_{g}=3.0,2.0$, and 1.0, respectively, in good agreement with the simulations! As the guide field decreases the turnover point moves further away from the X-line. For $B_{g}=0.35$, in agreement with Eq. 4.2 the turnover point is not within the plotted domain, but since a guide field is present, we expect to see a turnover point at distances further downstream than what our simulation box will allow. We also include a two-dimensional simulation for $B_{g}=3.0$. As expected, we 

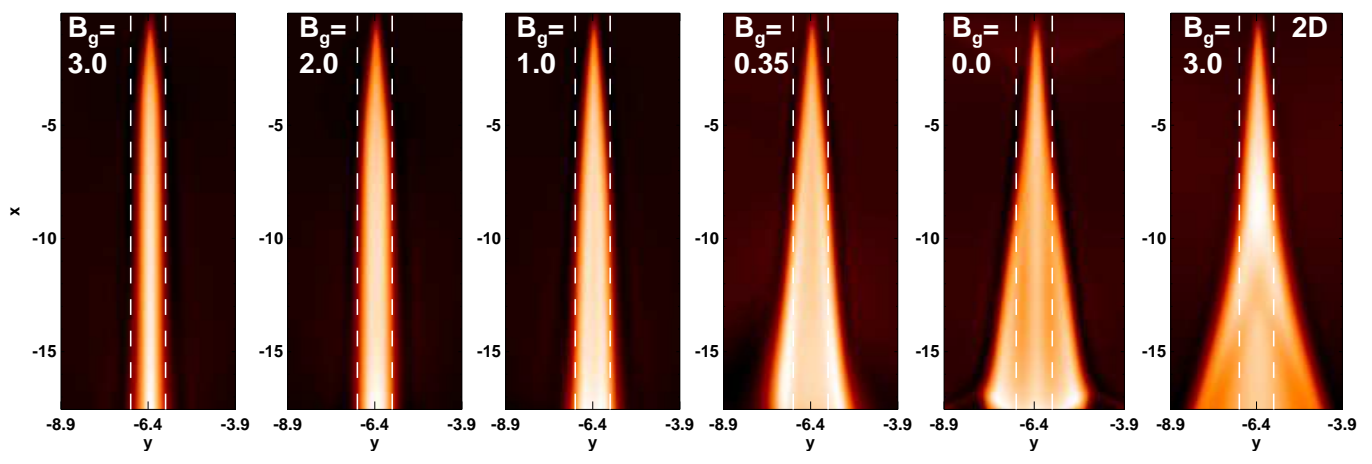

Figure 4.11: Collimation of exhaust vs. guide field.

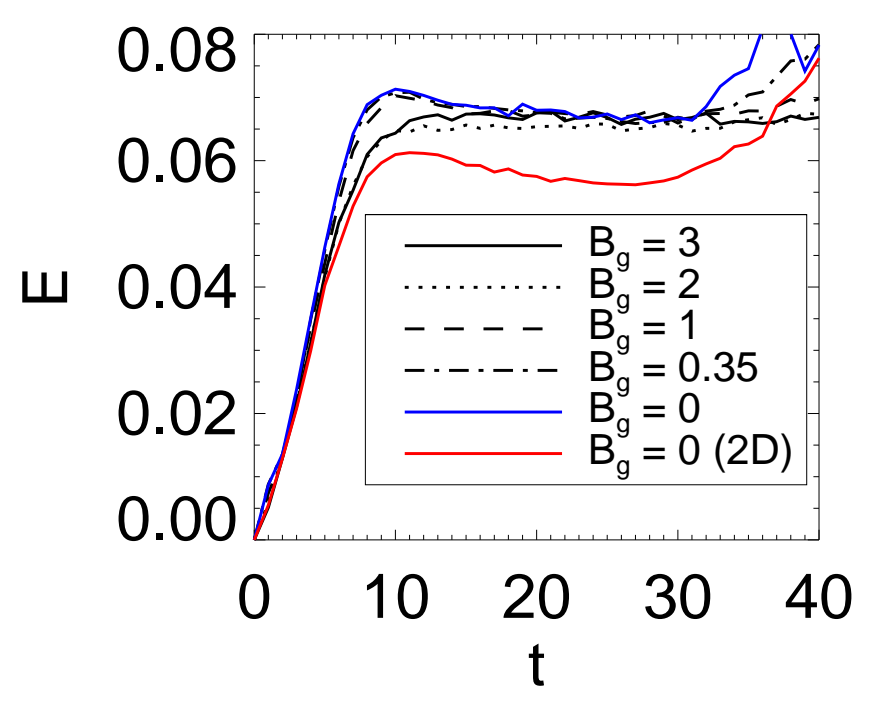

Figure 4.12: Reconnection rates for varying guide fields but for the same X-line length, $w_{0 z}$. Exhaust collimation does not affect the reconnection rates.

do not see any collimation of the exhaust confirming that the collimation effect is indeed a three-dimensional effect. In Fig. 4.12, the reconnection rate as a function of time is shown for each simulation from Fig. 4.11. We see here that that collimation of the exhaust does not affect the steady state reconnection rate. Therefore, while in 2D a collimated exhaust implies slow reconnection, the reconnection rate is still fast even with a thin exhaust in 3D!

The previous simulations held $w_{0 z}$ constant. If we vary $w_{0 z}$ and hold $B_{g}$ 


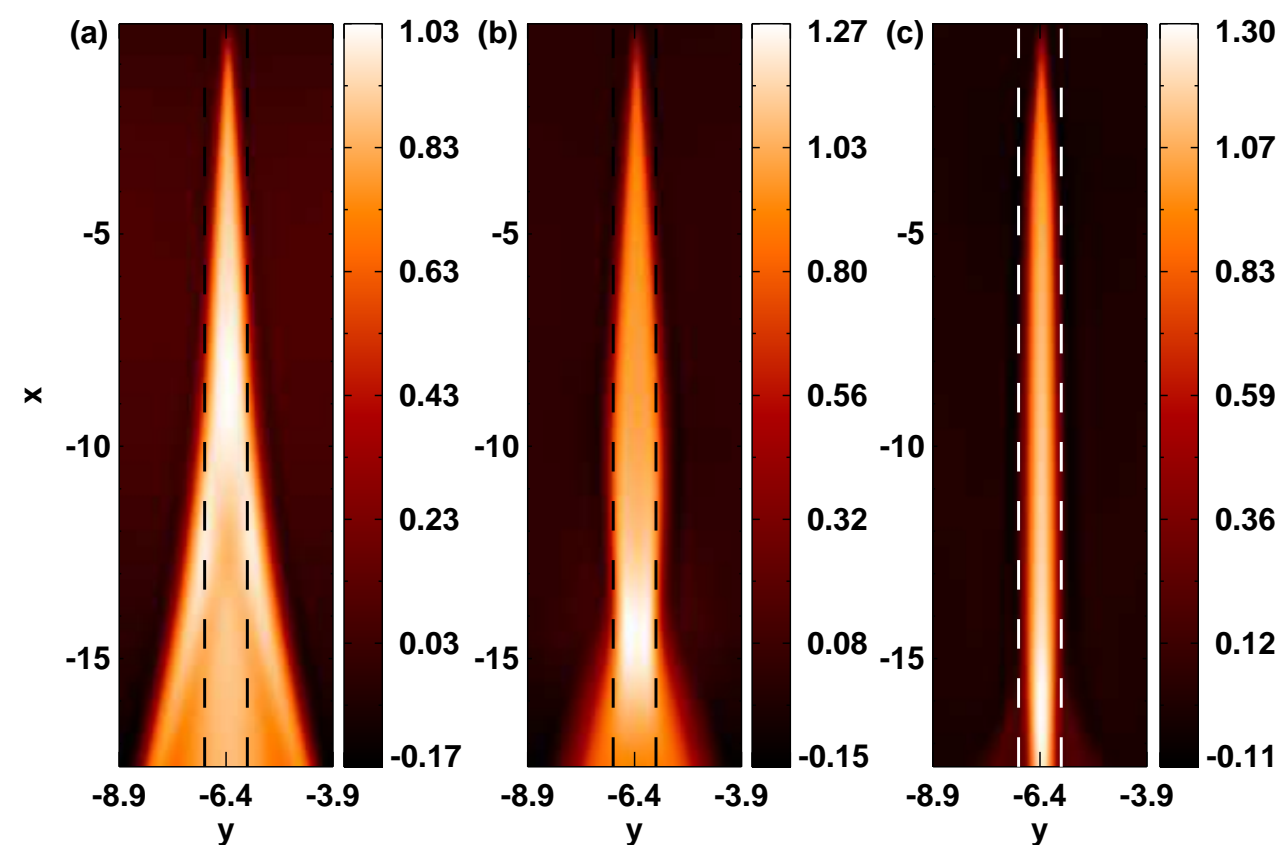

Figure 4.13: Collimation of exhaust vs. X-line length $w_{0 z}=(\mathrm{a}) 60$, (b) 30, and (c) 10.

constant, then according to Eq. 4.2 the turnover point will change accordingly. The reconnection outflow $v_{i x}$ is shown in Fig. 4.13 for $w_{0 z}=60,30$, and 10 in panels (a),(b), and (c), respectively. For reference, vertical dashed lines are added a distance of 0.5 from the center of the reconnection exhaust. The reconnection exhaust expands in the $y$-direction continuously in panel (a) because the turnover point is very far from the X-line, as expected from Eq. 4.2. As the length of the reconnection site decreases, the collimation of the exhaust approaches the X-line. For $B_{g}=3.0$, our simulation domain can support an X-line length $w_{0 z} \simeq 77$ before the turnover extends beyond the edge of the domain.

The collimation of the exhaust is a product of the rotational discontinuities passing through the $z=0$ plane. As discussed in Chapter 1, an important characteristic of RDs is there is a flow normal to the discontinuity. In Fig. 4.14(a), an image 


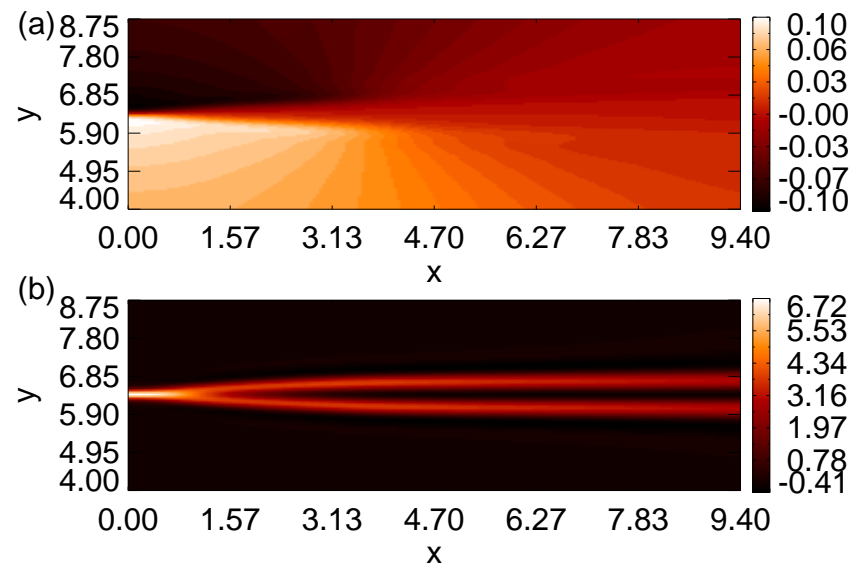

Figure 4.14: (a) Image of the inflow $V_{i y}$. The inflow terminates at the turnover point of the exhaust at $x \sim 3.3$. (b) The collimation of the exhaust can be seen in the image of the out-of-plane current $J_{z}$. The turnover is visible where the current stops expanding in the $y$-direction and lies parallel to the $x$-axis.

of the $y$-component of the ion velocity or inflow is shown for $B_{g}=3.0$ and $w_{0 z}=10$ in the $z=0$ plane. The inflow as expected is present along the exhaust in the $x$ direction until the turnover point near $x=10$, after which the exhaust collimates. Figure $4.14(\mathrm{~b})$ is an image of the out-of-plane current $J_{z}$; after collimation begins around $x=3.3$ the inflow $\left(v_{i y}\right)$ goes to zero. Indeed beyond the turnover point the exhaust is bounded by tangential discontinuities (TD) above and below. TDs do not allow particle flow across the discontinuity; in fact the current layer before reconnection occurs can be considered a tangential discontinuity. The RD appears to propagate until it reaches the turnover point and then transforms into a TD. However as discussed in Section 4.2, this is a geometric effect not a transformation.

We now look at the exhaust structure in the $y z$ plane to compare with the model proposed by Sasunov et al. (2012). Figure 4.15(a) is an image of the reconnection inflow $v_{i y}$ for the $B_{g}=3.0$ and $w_{0 z}=10$ simulation. The $y z$ cut is taken 

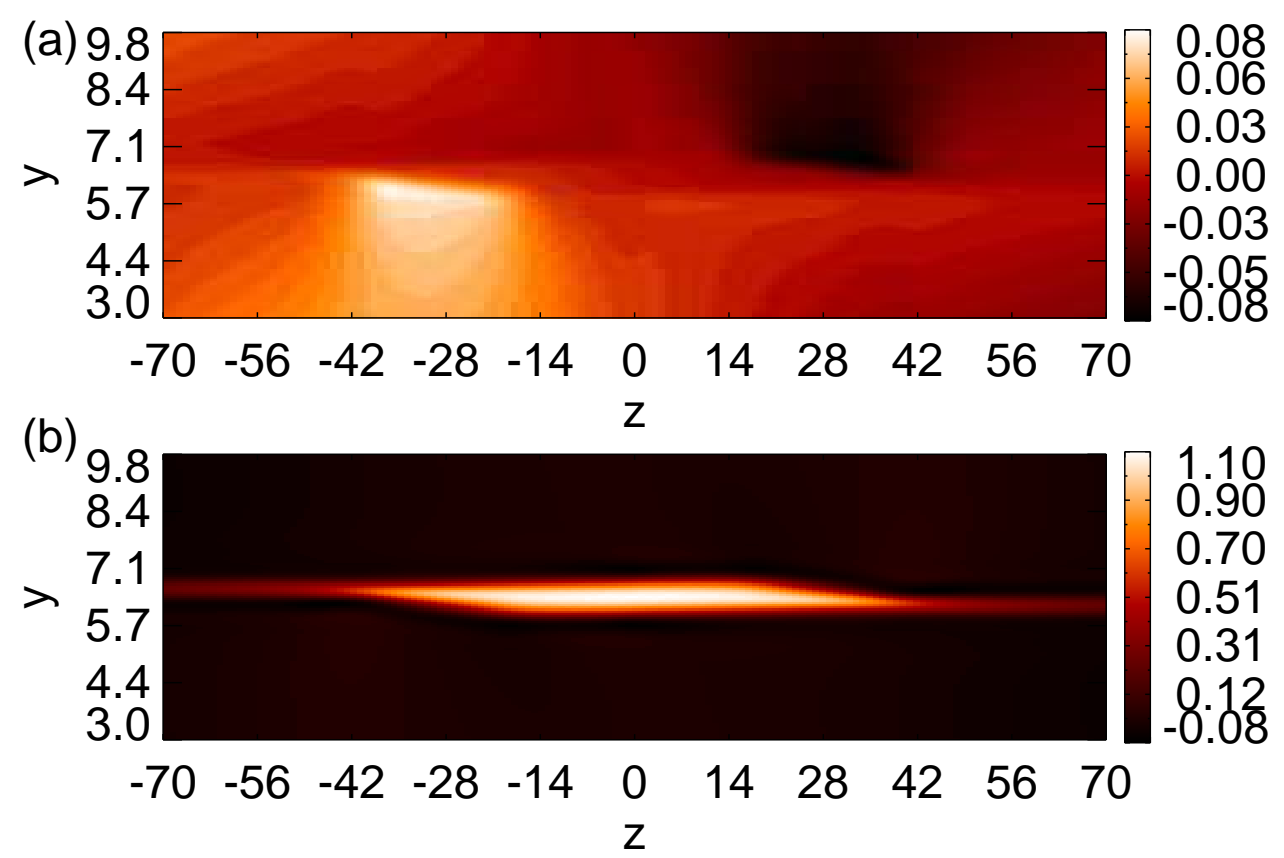

Figure 4.15: (a) Image of the inflow $V_{i y}$ in the $y z$-plane. The inflow indicates the presence of an RD. (b) Image of the reconnection exhaust in the $y z$-plane for $B_{g}=3.0$. The parallelogram structure is evident.

after the exhaust collimates in the $z=0$ plane. Two reconnection inflows are located around $z \sim-28$ and +28 , but not at $z=0$. This happens because the RDs propagate along reconnected field lines and leave the $z=0$ plane. We can envision the RDs forming a ribbon like structure which propagates from the $\mathrm{X}$-line in the outflow and out-of-plane direction. The top and bottom of the exhaust are TDs because there is no inflow across those boundaries. In Fig. 4.15(b), the parallelogram shape of the exhaust is clearly visible, as predicted by Sasunov et al. (2012)

The width of the reconnection exhaust for each simulation, measured at the turnover point as the half-width at half-max of $V_{i x}$ in the inflow direction as a function of $x$, is listed in Table 4.1. The columns of the table are the guide field $\left(B_{g}\right)$, the X-line length $\left(w_{0 z}\right)$, and the turnover point $\left(l_{x}\right)$. The "flux" column is the predicted 
width of the reconnection exhaust according to Eq. 4.5. The "geometry" column is calculated from the geometry argument given by Eq. 4.6. The measured exhaust widths agree well with the predicted widths from both flux and geometry arguments. In order to distinguish between the flux and geometry argument several simulations with small guide field would need to be performed. It should be noted that even though we do not observe a turnover point for some simulations we performed, we expect a turnover to occur for any system as long as there is a guide field and the Xline is localized. We only find a limited number of examples of exhaust collimation due to the finite size of our simulation domain and numerical constraints.

\begin{tabular}{|c|c|c|c|c|c|}
\hline$B_{g}$ & $w_{0 z}$ & $l_{x}$ & Flux & Geometry & Measured \\
\hline 3.0 & 10 & 3.3 & 0.44 & 0.47 & 0.60 \\
\hline 3.0 & 30 & 10.0 & 1.33 & 1.40 & 1.46 \\
\hline 2.0 & 10 & 5.0 & 0.63 & 0.70 & 0.79 \\
\hline 1.0 & 10 & 10.0 & 0.99 & 1.40 & 1.07 \\
\hline
\end{tabular}

\subsection{Applications}

\subsubsection{Solar Wind}

We have shown that localized magnetic reconnection can still have an impact outside of the region of reconnection in the presence of a guide field. The degree to which the reconnection exhaust expands into the out-of-plane direction depends on the strength of the guide field and width of the X-line. The magnetic reconnection 
observations made in the solar wind by Phan et al. (2006), where a reconnection exhaust of at least $390 R_{E}$ was observed, raises an important question. What is the cause of these large scale reconnection exhausts? There are two possible explanations for this observation. The first option is reconnection begins at small scales near the sun, and as reconnection convects toward Earth, reconnection spreads into the out-of-plane direction. The second possibility is that reconnection is finite and the exhaust (not the reconnection) spreads into the out-of-plane direction. Both of these scenarios will be discussed in the following few paragraphs.

One can ask whether the spreading of reconnection in the out-of-plane direction could allow the X-line to be on the order of hundreds of $R_{E}$. We can estimate the size of an X-line. We assume that reconnection begins close to the Sun with an initially small finite X-line length. Suppose the reconnection site convects out with the solar wind at a speed $v_{S W}$. (For simplicity, this calculation ignores variations in solar wind speed, magnetic field strength, and plasma density as a function of distance from the Sun.) Then, the time it takes to get to a position $r_{f}$ away from the Sun is $t \sim r_{f} / v_{S W}$. If the speed of the spreading of the $\mathrm{X}$-line is $v_{X}$, then the extent $L$ of the X-line at $r_{f}$ is $L \sim v_{X} t \sim r_{f} v_{X} / v_{S W}$, which gives the upper limit on the length of the X-line that could arise in the solar wind.

One can test the implications of this from the observations of the reconnection event in the study [Phan et al. (2006)], where the solar wind speed is inferred to be $v_{S W}=340 \mathrm{~km} / \mathrm{s}$. The satellite observations occurred near the Earth, so $r_{f} \simeq 1 \mathrm{AU} \simeq 2.3 \times 10^{4} R_{E}$. The Alfvén speed based on a guide field of strength $B_{g}=4 \mathrm{nT}$ and density $n=20 \mathrm{~cm}^{3}$ is $c_{A g}=19 \mathrm{~km} / \mathrm{s}$. If we take this as the 

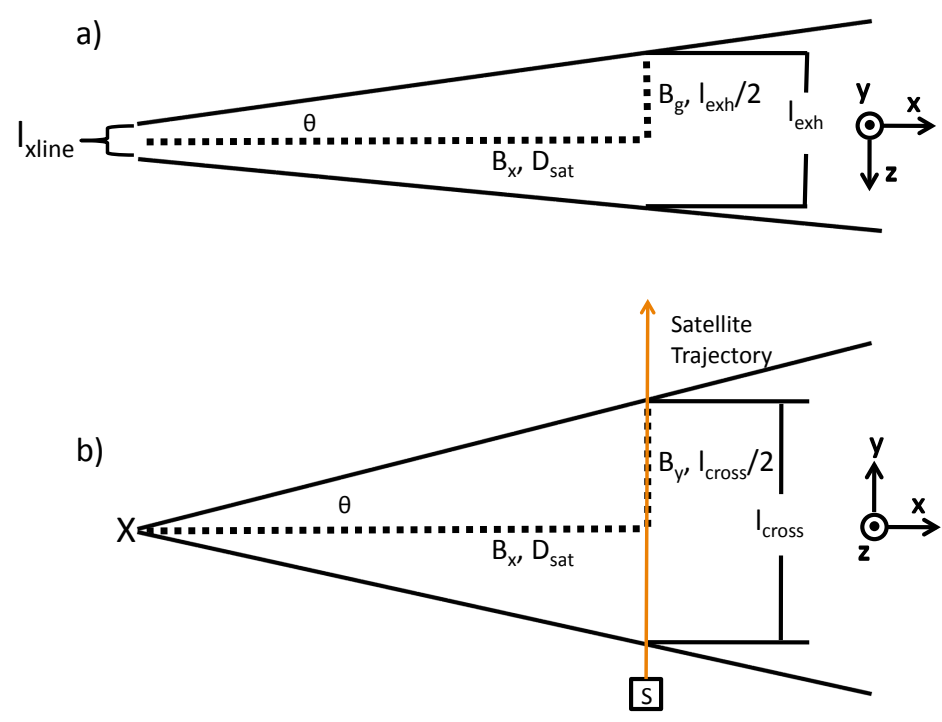

Figure 4.16: (a) Sketch of the reconnection exhaust in the $x z$ plane. (b) Sketch of the reconnection exhaust in they $x y$ plane.

spreading speed $v_{X}$, then the maximum length of the $\mathrm{X}$-line is $L \sim r_{f} v_{X} / v_{S W} \sim$ $1.3 \times 10^{3} R_{E}$. This exceeds the length of the X-line reported by Phan et al. (2006), which was $390 R_{E}$. In this event, the strength of the guide field was 0.35 of the reconnecting field, so the Alfvén speed is the slower of the two velocities and the extent of the X-line if spreading is due to current carriers is even longer. Thus, while this calculation assumes that the reconnection proceeds at short distance from the Sun, the calculation gives an indication that it is not impossible to achieve reconnection X-lines of the lengths reported by Phan et al. (2006), but this does not point to spreading as the only mechanism.

To predict the length of the X-line, consider the distance the observing satellites $\left(D_{\text {sat }}\right)$ are from the X-line to provide some clarity on how large scale structures in the solar wind may be formed. Figure 4.16(a) displays a sketch of the $x z$-plane structure of the reconnection exhaust. The solid black lines mark the edge of the 
exhaust. How far away must the satellites be to measure an exhaust of length $l_{\text {exh }}$ of $390 R_{E}$ ? The solid black lines reference the boundary of the exhaust. In a localized reconnection scenario, we calculate $D_{\text {sat }}$ from the geometry of the magnetic field where $\tan (\theta)=\left(l_{\text {exh }}-l_{\text {xline }}\right) / 2 D_{\text {sat }}=B_{g} / B_{x}$. We find that

$$
D_{s a t}=\frac{\left(l_{\text {exh }}-l_{x l i n e}\right) B_{x}}{2 B_{g}}
$$

We now measure the satellite distance $D_{\text {sat }}$ using the observation data from Phan et al. (2006). A sketch of how the measurement is made is shown in Fig. 4.16(b), where $l_{\text {cross }}$ is determined by the speed of the satellite divided by the crossing time of the satellite through the reconnection exhaust. Using the geometry of the in-plane magnetic field, we find $\tan (\theta)=l_{\text {cross }} / D_{\text {sat }}=B_{y} / B_{x}$. Solving for $D_{\text {sat }}$ yields

$$
D_{\text {sat }}=l_{\text {cross }} \frac{B_{x}}{B_{y}},
$$

$B_{y}$ is the strength of the magnetic field in the inflow direction. We find the distance $D_{\text {Sat }} \sim 270 R_{E}$. Solving Eq. 4.9 for the X-line length gives

$$
l_{\text {xline }}=l_{\text {exh }}-\frac{2 B_{g} D_{\text {Sat }}}{B_{x}} .
$$

For the parameters in the Phan et al. (2006) event, we find $l_{x l i n e} \geq 200 R_{E}$. The minimum length of the $3 \mathrm{D}$ X-lines tends to be on the order of $10 d_{i}$ [Shay et al. (2003)]. The ion inertial length in the solar wind during the Phan et al. (2006) event is found to be $d_{i} \sim 50 \mathrm{~km} \sim 8 \times 10^{-3} R_{E}$. Therefore, the X-line responsible 
for this exhaust is at least hundreds of $R_{E}$ long, this is significantly larger than the expected minimum length of $10 d_{i}$ [Shay et al. (2003)]. Note, the thickness of the exhaust is assumed to not be collimated.

\subsubsection{Supra-Arcade Downflows}

Supra-arcade downflows (SADs) are observed in the solar corona as dark patches that descend towards the solar surface during solar flares [McKenzie \& Hudson (1999); McKenzie (2000)] and are highlighted in Fig. 4.2. SADs are interpreted as a density depletion in the high density plasma of the arcade, and because they are comparatively thin and have a curved shape, they are often called "tadpoles". SADs are generally thought of as reconnected flux tubes that are contracting under tension [McKenzie \& Hudson (1999); McKenzie (2000); Asai et al. (2004); Savage et al. (2010); Warren et al. (2011)]. Simulations of SADs [Linton \& Longcope (2006)] produced flux tubes with short-lived patchy reconnection which led to a teardrop-shaped cross section. A recent study by Savage et al. (2012) posits SADs are not contracting flux tubes, but the wake of the flux tube caused passing through the plasma. A recent work [Cassak et al. (2013)] suggests that SADs are due to reconnection that is persistent in time (as opposed to short-lived or bursty).

An issue with the idea that persistent reconnection explains SADs is that the exhaust in $2 \mathrm{D}$ reconnection opens out in order to be fast, so this would produce wide cavities, instead of the thin tadpole-like voids that are observed. However, we have shown that localized reconnection remains collimated. This potentially shows how 


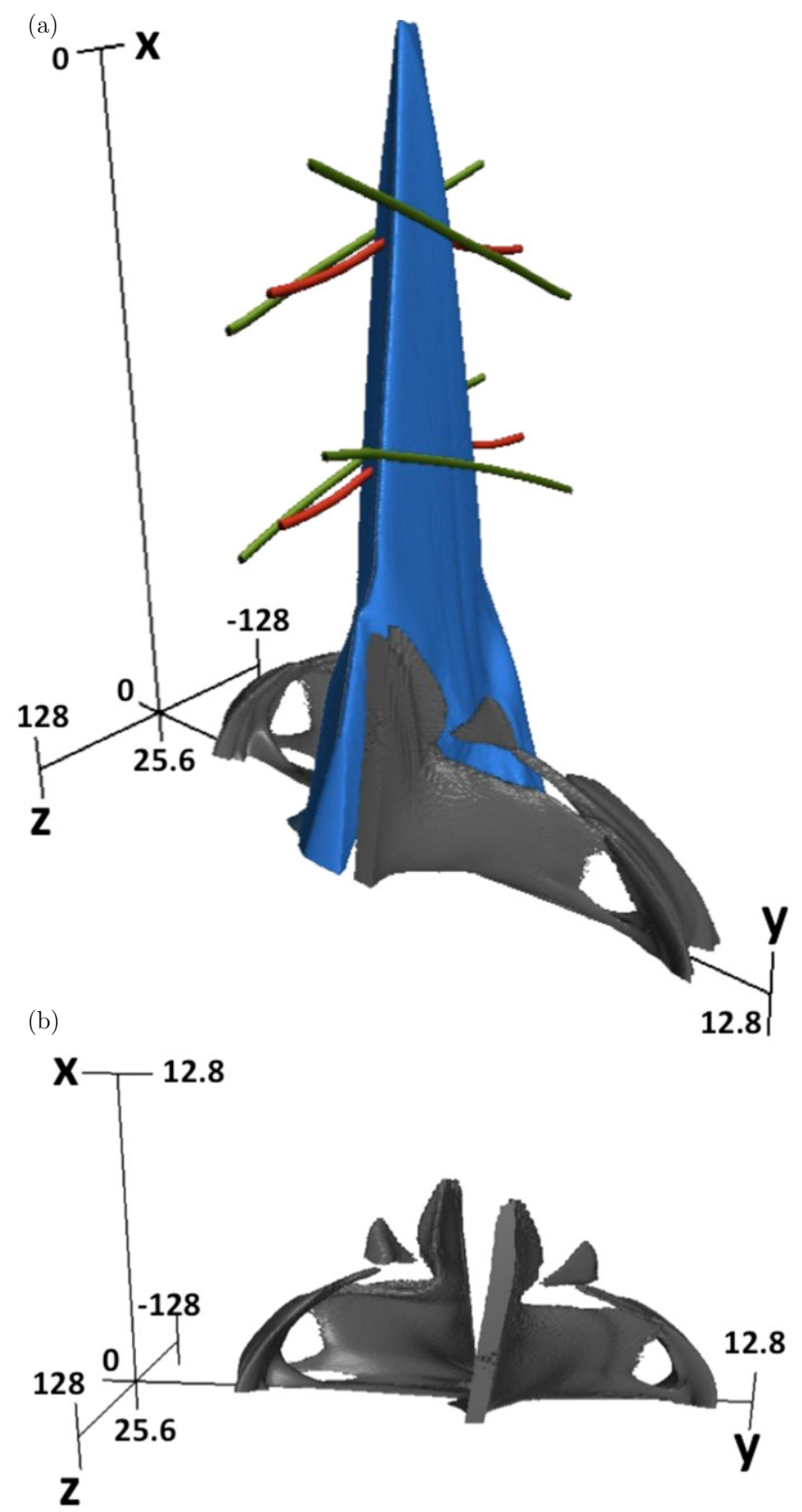

Figure 4.17: (a) 3D view of the reconnection exhaust (blue isosurface) and density (gray isosurface), showing that the exhaust collimates in the $y$ direction but spreads in the $z$ direction. Notice, the exhaust creates a density depletion in the primary island (analogous to an arcade). Non-reconnected field lines are shown in red and reconnected field lines are shown in green. (b) The density isosurface from panel (a) is rotated to reveal the $3 \mathrm{D}$ SAD-like density depletion. 
thin SADs are carved out by reconnection exhausts - if the reconnection is localized. To see this, Fig. 4.17(a) shows the three-dimensional structure of the reconnection exhaust $\left(v_{i x}\right)$, represented by the blue isosurface, for the simulation $w_{0 z}=10$ and $B_{g}=3.0$. Here, the green lines are non-reconnected field lines and the reconnected field lines are shown in red. The limited expansion of the exhaust into the inflow $(y)$ direction and the large expansion in the out-of-plane $(z)$ direction is clearly visible. The grey surface is high density plasma in an island (analogous to a solar arcade) populated by reconnection. In Fig. 4.17(b) the exhaust isosurface is removed and the density is rotated around the $x$-axis and a very thin cavity is carved out of the high density plasma, and this cavity is very similar in appearance to SADs. Therefore, localized reconnection can produce thin density cavities in high density regions and may be important in the formation of SADs.

\subsection{Conclusion}

We studied the effect of localization of magnetic reconnection on the reconnection exhaust. Even though the reconnection is localized, the exhaust can still expand into the out-of-plane direction in the presence of a guide field. This fact is important for observations of reconnection in the solar wind. It is feasible to get large scale reconnection structures through spreading and from localized reconnection. We believe that in the case of the Phan et al. (2006) event of a large reconnection exhaust structure in the solar wind is the by-product of an extended X-line $\left(l_{\text {xline }} \geq 200 R_{E}\right)$, by Eq. 4.11. However, we have shown small scale X-lines 
can produce large scale structures if there is a guide field. Therefore, an extended exhaust does not imply an extended X-line.

We also discussed the collimation of magnetic reconnection exhausts when the magnetic reconnection is localized and remains localized. It is expected that the exhaust collimates as long as there is a guide field present and the reconnection cannot spread. Localized reconnection with a guide field appears to be a very important component of supra-arcade downflows (SADs), and due to collimation of the exhaust of localized reconnection can carve thin, low density cavities in the high density magnetic islands. The collimation of the exhaust is caused by the geometry of the magnetic field. 


\section{Chapter 5}

\section{Summary of Work}

\subsection{Summary of Results}

In summary, magnetic reconnection is a plasma process in which stored magnetic energy is converted into thermal and kinetic energies of the surrounding plasma. Oppositely directed magnetic field lines break and cross connect due to a dissipative mechanism (e.g., resistivity). The now bent, reconnected field lines retreat from the X-line (the point of reconnection) at the Alfvén speed due to the magnetic tension in the reconnected magnetic field, therefore generating outflows. The plasma leaving the reconnection site creates a region of low pressure, which in turn pulls in more plasma that pulls in more magnetic fields. The new magnetic fields that enter the region can now also break and reconnect. Reconnection can continue in a steady-state. The process of reconnection is believed to be responsible for the rapid energy release in solar flare events and geomagnetic substorms.

Magnetic reconnection can persist in various different regimes. The slowest form of reconnection is Sweet-Parker reconnection, which is too slow to explain observations. Sweet-Parker enhanced by secondary islands is faster than SweetParker reconnection rates and believed by some to explain energy release rates in the corona. The regime of reconnection that can explain observed energy release rates is Hall reconnection. 
This dissertation addresses three fundamental properties of magnetic reconnection. The first is the role of secondary islands in energy release, essentially can secondary islands explain the energy release rates in solar explosive events? We then transitioned from 2D magnetic reconnection into studying 3D dynamics of magnetic reconnection, more specifically exploring how localized reconnection spreads into the out-of-plane direction and the conditions responsible for the transition between two different spreading mechanisms.

The final topic involves the structure of localized magnetic reconnection, and a study into whether localized reconnection occurs in the solar wind and the formation of supra-arcade downflows (SADs). We summarize the results in the following sections.

\subsubsection{Role of Secondary Islands in Energy Release}

We studied the transition from Sweet-Parker reconnection to Sweet-Parker reconnection enhanced by secondary islands to Hall reconnection; the first simulation to separate the three regimes of reconnection. Three main results from this study are discovered. First, there exists a regime of reconnection with secondary islands but without the Hall effect playing a significant role. Second, secondary island reconnection is faster than Sweet-Parker, but still much slower than Hall reconnection. This implies that secondary islands are not the cause of the fastest reconnection rates. Thirdly, the onset of Hall reconnection ejects secondary islands in the vicinity of the X-line. This all points towards the notion that Hall reconnection is the most 
efficient form of energy release.

This study made some assumptions that will require further study. These omissions include: ohmic heating, viscosity, Dreicer field effects, a guide field, threedimensional effects, and employing a Spitzer resistivity. Future research involving solar flare energy release will be discussed in the next section.

\subsubsection{Guide Field Dependence of X-line Spreading}

We studied the guide field dependence of 3D X-line spreading during collisionless magnetic reconnection. Theoretical arguments are used to predict the strength of the guide field at which a transition from current carrying spreading to Alfvén wave spreading occurs. In the weak guide field limit, spreading is due to the motion of the current carriers. Spreading in the strong guide field limit is bidirectional and is due to the excitation of Alfvén waves along the guide field. We hypothesize that the X-line spreads bidirectionally with a speed governed by the faster of the two mechanisms for each direction. A prediction on the strength of the guide field $\left(B_{g}=2.0\right)$ when this transition occurs is found and tested with 3D simulations. When the guide field is weaker than $B_{g}=2.0$, the X-line spreads in the out-of-plane direction by the current carriers (electrons for our study). Above the predicted guide field strength, the X-line spreads in the out-of-plane direction by Alfvén waves.

A few assumptions are made in the formation of this work. We treat our system as quasi-2D, meaning any variation in the system in the direction of the current is negligible. The current sheets in all simulations performed are initially 
thin, meaning that free magnetic energy has already been stored. The plasma parameters across the current sheet are assumed symmetric. The simulations employ a two-fluid model, which does not fully capture electron scale physics. This may make quantitative changes to our results (such as the estimate of $\delta$ and the size of $\left.B_{r e c}\right)$, but we do not expect qualitative changes to the theoretical results. The simulations are isothermal and contain no thermal conduction.

Another main assumption is that only a single mode is dominating the dynamics. However, the role of multiple oblique modes can play an important part of the dynamics of the spreading process. As seen in our simulations, the X-line structure is identifiable at early times but as the complicated nature of the oblique modes develop, the X-line structure breaks up due to the interaction between the current sheet. The interaction of oblique modes can impede X-line spreading [Schreier et al. (2010)]. More work is necessary on the impact oblique modes have on X-line spreading.

Future work would involve employing resistive-Hall MHD simulations for 3D magnetic reconnection. An unanswered question during solar flares is how does the energy get stored prior to the rapid energy release during the flare? Generally, Sweet-Parker can be thought of as an energy storage phase of reconnection and then a transition to fast reconnection releases this stored energy. In a few test simulations, we found that Sweet-Parker reconnection does not spread in the out-ofplane direction. This could have important implications on pre-flare energy storage, and lead to a better understanding of the flaring cycle. 


\subsubsection{X-line Structure for Localized Reconnection}

We studied the effect of localizing magnetic reconnection in the out-of-plane direction by using an anomalous resistivity. We found that in the presence of a guide field, the reconnection exhaust can expand into the out-of-plane direction forming a ribbon-like structure. The opening angle of the ribbon is dependent on the strength of the guide field. The exhaust is bounded by four MHD-discontinuities. Two RDs allow plasma to enter the reconnection exhaust and are caused by reconnected field lines. The other boundaries are TDs which are caused by unreconnected layers of magnetic field that bound the collimated portion of the reconnection exhaust.

The expansion of the reconnection exhaust is relevant to the solar wind, and the collimation of the exhaust may be vital in the formation of supra-arcade downflows (SADs). The solar wind observation from Phan et al. (2006) of a reconnection exhaust $390 R_{E}$ long is believed to be a product of an extended X-line and not reconnection being localized. Calculation of the length of the X-line reveal that the Phan et al. (2006) event was at least $200 R_{E}$ in length. Cassak et al. (2013) claims that the SADs require magnetic reconnection to be localized. If the exhausts spread in time, the density carved out by the reconnection exhaust would not be long, thin structures but large swaths of darkening.

This model localized the reconnection with an anomalous resistivity and neglected the Hall term. Future work would include localizing the reconnection by the magnetic field geometry by fluting the current sheet (e.g., making the current sheet thinner near the middle of the out-of-plane simulation domain and wider at the 
edges) and including the Hall term, thus reintroducing spreading effects. This could have applications for sympathetic flares, which are flares that are triggered by the disruption of the magnetic field in the upper corona caused by other flares. A single flare has been observed triggering nearby explosive events [Török et al. (2011)]. Creating multiple fluted current sheets in the same simulation domain could address sympathetic flares. 


\section{Bibliography}

Asai A., Shibata K., Yokoyama T., Shimogo M., 2004, in Sakurai T., Sekii T., eds, Solar-B Mission and the Forefront of Solar Physics Vol. 325 of Astronomical Society of the Pacific Conference Series, Downflow as a reconnection outflow. p. 361

Baalrud S. D., Bhattacharjee A., Huang Y. M., 2012, Phys. Plasmas, 19, 022101

Baalrud S. D., Bhattacharjee A., Huang Y.-M., Germaschewski K., 2011, Phys. Plasmas, 18, 092108

Bhattacharjee A., 2004, Annu. Rev. Astron. Astrophys., 42, 365

Bhattacharjee A., Huang Y.-M., Yang H., Rogers B., 2009, Phys. Plasmas, 16, 112102

Birn J., Drake J. F., Shay M. A., Rogers B. N., Denton R. E., Hesse M., Kuznetsova M., Ma Z. W., Bhattacharjee A., Otto A., Pritchett P. L., 2001, J. Geophys. Res., 106,3715

Biskamp D., 1986, Phys. Fluids, 29, 1520

Carrington R. C., 1859, MNRAS, 20, 13

Cassak P. A., 2006, PhD thesis, University of Maryland, www.physics.udel.edu/ ${ }^{\sim}$ pcassak/cassakthesis.pdf

Cassak P. A., Drake J. F., 2009, Ap. J. Lett., 707, L158

Cassak P. A., Drake J. F., Gosling J. T., Phan T.-D., Shay M. A., Shepherd L. S., 2013, The Astrophysical Journal Letters, 775, L14

Cassak P. A., Drake J. F., Shay M. A., 2006, Ap. J., 644, L145

Cassak P. A., Drake J. F., Shay M. A., Eckhardt B., 2007, Phys. Rev. Lett., 98, 215001

Cassak P. A., Mullan D. J., Shay M. A., 2008, Ap. J. Lett., 676, L69

Cassak P. A., Shay M. A., 2007, Phys. Plasmas, 14, 102114

Cassak P. A., Shay M. A., Drake J. F., 2005, Phys. Rev. Lett., 95, 235002

Cassak P. A., Shay M. A., Drake J. F., 2009, Phys. Plasmas, 16, 102702

Cassak P. A., Shay M. A., Drake J. F., 2010, Phys. Plasmas, 17, 062105,

Ciaravella A., Raymond J. C., 2008, Ap. J., 686, 1372 
Cothran C. D., Landreman M., Brown M. R., Matthaeus W. H., 2005, Geophys. Res. Lett., 32, L03105

Daughton W., Roytershteyn V., 2011, Space Sci. Rev., doi:10.1007/s11214-011-9766$\mathrm{Z}$

Daughton W., Roytershteyn V., Albright B. J., Karimabadi H., Yin L., Bowers K. J., 2009a, Phys. Plasmas, 16, 072117

Daughton W., Roytershteyn V., Albright B. J., Karimabadi H., Yin L., Bowers K. J., 2009b, Phys. Rev. Lett., 103, 065004

Daughton W., Scudder J., Karimabadi H., 2006, Phys. Plasmas, 13, 072101

Dorfman S. E., 2012, PhD thesis, Princeton University

Drake J. F., Swisdak M., Che H., Shay M. A., 2006, Nature, 443, 553

Drake J. F., Swisdak M., Schoeffler K. M., Rogers B. N., Kobayashi S., 2006, Geophys. Res. Letter, 33, L13105

Drake J. F., Swisdak M., Shay M. A., Rogers B. N., Zeiler A., Cattell C., 2003, Science, 299, 873

Dungey J. W., 1953, Phil. Mag., 44, 725

Dungey J. W., 1958, Cosmic Electrodynamics. Cambridge University Press

Egedal J., Katz N., Bonde J., Fox W., Le A., Porkolab M., Vrublevskis A., 2011, Phys. Plasmas, 18, 111203

Fermo R. L., Drake J. F., Swisdak M., 2010, Phys. Plasmas, 17, 010702

Fermo R. L., Drake J. F., Swisdak M., 2012, Phys. Rev. Lett., 108, 255005

Fuselier S. A., Frey H. U., Trattner K. J., Mende S. B., Burch J. L., 2002, J. Geophys. Res., 107, 1111

Gosling J. T., Eriksson S., Blush L. M., Phan T. D., Luhmann J. G., McComas D. J., Skoug R. M., Acuna M. H., Russell C. T., Simunac K. D., 2007, Geophysical Research Letters, 34, n/a

Grasso D., Borgogno D., Pegoraro F., 2007, Phys. Plasmas, 14, 055703

Hodgson R., 1859, MNRAS, 20, 15

Huang Y.-M., Bhattacharjee A., 2010, Phys. Plasmas, 17, 062104

Huang Y.-M., Bhattacharjee A., Sullivan B. P., 2011, Phys. Plasmas, 18, 072109

Huba J. D., Gladd N. T., Papadopoulos K., 1977, Geophys. Res. Lett., 4, 125 
Huba J. D., Rudakov L. I., 2002, Phys. Plasmas, 9, 4435

Huba J. D., Rudakov L. I., 2003, Phys. Plasmas, 10, 3139

Huba J. D., Rudakov L. I., 2004, Phys. Rev. Lett., 93, 175003

Isobe H., Yokoyama T., Shimojo M., Morimoto T., Kozu H., Eto S., Narukage N., Shibata K., 2002, Ap. J., 566, 528

Jemella B. D., Shay M. A., Drake J. F., Rogers B. N., 2003, Phys. Rev. Lett., 91, 125002

Karimabadi H., Krauss-Varban D., Huba J. D., Vu H. X., 2004, J. Geophys. Res., 109, A09205

Katz N., Egedal J., Fox W., Le A., Bonde J., Vrublevskis A., 2010, Phys. Rev. Lett., 104,255004

Kliem B., 1995, in Benz A. O., Krueger A., eds, Proc. CESRA Workshop Vol. 444, Coupled magnetohydrodynamic and kinetic development of current sheets in the solar corona. p. 93

Kowal G., Lazarian A., Vishniac E. T., Otmianowska-Mazur K., 2009, Ap. J., 700, 63

Kulsrud R. M., 2001, Earth, Planets, and Space, 53, 417

Landau L. D., Lifshitz E. M., 1960, Electrodynamics of continuous media

Lapenta G., 2008, Phys. Rev. Lett., 100, 235001

Lapenta G., Krauss-Varban D., Karimabadi H., Huba J. D., Rudakov L. I., Ricci P., 2006, Geophys. Res. Lett., 33, L10102

Lazarian A., Vishniac E., 1999, Ap. J., 517, 700

Levy R. H., Petschek H. E., Siscoe G. L., 1964, AIAA J., 2, 2065

Lin J., Li J., Ko Y.-K., Raymond J. C., 2009, Ap. J., 693, 1666

Lin Y., Lee L. C., 1993, Space Sci. Rev., 65, 59

Linton M. G., Longcope D. W., 2006, Ap. J., 642, 1177

Liu R., Wang H. M., 2010, Ap. J. Lett., 714, 41

Liu R., Wang H. M., Alexander D., 2009, Ap. J., 696, 121

Longcope D. W., McKenzie D. E., Cirtain J., Scott J., 2005, Ap. J., 630, 596

Loureiro N. F., Samtaney R., Schekochihin A. A., Uzdensky D. A., 2012, Physics of Plasmas, 19, 042303 
Loureiro N. F., Schekochihin A. A., Cowley S. C., 2007, Phys. Plasmas, 14, 100703

Loureiro N. F., Uzdensky D. A., Schekochihin A. A., Cowley S. C., Yousef T. A., 2009, Mon. Not. R. Astron. Soc., 399, L146

Lukin V. S., Linton M. G., 2011, Nonlinear Processes in Geophysics, 18, 871

Ma Z. W., Bhattacharjee A., 1996, Geophys. Res. Lett., 23, 1673

McKenzie D. E., 2000, Solar Phys., 195, 381

McKenzie D. E., Hudson H. S., 1999, Ap. J., 519, L93

McPherron R. L., Russell C. T., Aubry M. P., 1973, J. Geophys. Res., 78, 3131

Malakit K., Cassak P. A., Shay M. A., Drake J. F., 2009, Geophys. Res. Lett., 36, L07107

Mandt M. E., Denton R. E., Drake J. F., 1994, Geophys. Res. Lett., 21, 73

Matthaeus W. H., Lamkin S. L., 1985, Phys. Fluids, 26, 303

Matthaeus W. H., Lamkin S. L., 1986, Phys. Fluids, 29, 2513

Mozer F., Bale S. D., Phan T. D., 2002, Phys. Rev. Lett., 89, 015002

Nagai T., 1982, J. Geophys. Res., 87, 4405

Nagai T., Shinohara I., Zenitani S., Nakamura R., Nakamura T. K. M., Fujimoto M., Saito Y., Mukai T., 2013, Journal of Geophysical Research (Space Physics), 118,1667

Nakamura T. K. M., Fujimoto M., Sekiya H., 2010, Geophys. Res. Lett., 37, L02103

Nakamura T. K. M., Nakamura R., Alexandrova A., Kubota Y., Nagai T., 2012, J. Geophys. Res., 117, 03220

Øieroset M., Phan T. D., Fujimoto M., Lin R. P., Lepping R. P., 2001, Nature, 412, 417

Parker E. N., 1957, J. Geophys. Res., 62, 509

Petschek H. E., 1964, in Ness W. N., ed., AAS/NASA Symposium on the Physics of Solar Flares Magnetic field annihilation. NASA, Washington, DC, p. 425

Phan T. D., Gosling J. T., Davis M. S., Skoug R. M., Oieroset M., Lin R. P., Lepping R. P., McComas D. J., Smith C. W., Reme H., Balogh A., 2006, Nature, 439, 175 
Phan T.-D., Kistler L. M., Klecker B., Haerendel G., Paschmann G., Sonnerup B. U. O., Baumjohann W., Bavassano-Cattaneo M. B., Carlson C. W., Dilellis A. M., Fornacon K. H., Frank L. A., Fujimoto M., Georgescu E., Kokubun S., Moebius E., Mukai T., Oieroset M., Paterson W. R., Reme H., 2000, Nature, 404, 848

Qiu J., 2009, Ap. J., 692, 1110

Qiu J., Liu W., Hill N., Kazachenko M., 2010, Ap. J., 725, 319

Ren Y., Yamada M., Gerhardt S., Ji H., Kulsrud R., Kuritsyn A., 2005, Phys. Rev. Lett., 95, 005003

Rogers B. N., Denton R. E., Drake J. F., Shay M. A., 2001, Phys. Rev. Lett., 87, 195004

Rossi B., Olbert S., 1970, Introduction to the Physics of Space. McGraw-Hill Book Company

Runov A., Nakamura R., Baumjohann W., Treumann R. A., Zhang T. L., Volwerk M., Vörös Z., Balogh A., Glabmeier K.-H., Klecker B., Réme H., Kistler L., 2003, Geophys. Res. Lett., 30, 1579

Sasunov Y. L., Semenov V. S., Heyn M. F., Kubyshkin I. V., Biernat H. K., 2012, Geophysical Research Letters, 39, 6104

Sato T., Hayashi T., 1979, Phys. Fluids, 22, 1189

Savage S. L., McKenzie D. E., Reeves K. K., 2012, Ap. J. Lett., 747, L40

Savage S. L., McKenzie D. E., Reeves K. K., Forbes T. G., Longcope D. W., 2010, Ap. J., 722, 329

Scholer M., 1989, J. Geophys. Res., 94, 8805

Schreier R., Swisdak M., Drake J. F., Cassak P. A., 2010, Physics of Plasmas, 17, 110704

Scudder J. D., Mozer F. S., Maynard N. C., Puhl-quinn P. A., Ma Z. W., Russell C. T., 2002, J. Geophys. Res., 107, 2002

Shay M. A., Drake J. F., Rogers B. N., Denton R. E., 1999, Geophys. Res. Lett., 26,2163

Shay M. A., Drake J. F., Swisdak M., Dorland W., Rogers B. N., 2003, Geophys. Res. Lett., 30, 1345

Shay M. A., Drake J. F., Swisdak M., Rogers B. N., 2004, Phys. Plasmas, 11, 2199

Shepherd L. S., Cassak P. A., 2010, Phys. Rev. Lett., 105, 015004 
Shepherd L. S., Cassak P. A., 2012, J. Geophys. Res., 117, A10101

Shepherd L. S., Cassak P. A., Drake J. F., Gosling J. T., Phan T.-D., 2014, In Preparation

Shi Y., Lee L. C., 1990, Planet. Space Sci., 38, 437

Shibata K., Tanuma S., 2001, Earth Planets Space, 53, 473

Skender M., Lapenta G., 2010, Phys. Plasmas, 17, 022905

Smith D., Ghosh S., Dmitruk P., Matthaeus W. H., 2004, Geophys. Res. Lett., 31, L02805

Sonnerup B. U. Ö., 1979, in Lanzerotti L. J., Kennel C. F., Parker E. N., eds, Solar System Plasma Physics Vol. 3, Magnetic field reconnection. North Halland Pub., Amsterdam, p. 46

Speiser T. W., 1970, Planet. Space Sci., 18, 613

Sweet P. A., 1958, in Lehnert B., ed., Electromagnetic Phenomena in Cosmical Physics The neutral point theory of solar flares. Cambridge University Press, New York, p. 123

Török T., Panasenco O., Titov V. S., Mikić Z., Reeves K. K., Velli M., Linker J. A., Toma G. D., 2011, Ap. J. Lett., 739, L63

Tripathi D., Isobe H., Mason H. E., 2006, Astron. Astrophys., 453, 1111

Uzdensky D. A., 2007, Ap. J., 671, 2139

Uzdensky D. A., Loureiro N. F., Schkochihin A. A., 2010, Phys. Rev. Lett., 105, 235002

Vasyliunas V. M., 1975, Rev. Geophys., 13, 303

Warren H. P., O’Brien C. M., Neil R. Sheeley J., 2011, Ap. J., 742, 92

Zakharov L., Rogers B., Migliuolo S., 1993, Phys. Fluids B, 5, 2498 